\title{
Persistence of Illusion Using spatial illusion as a visual performance mechanism
}

\section{Johann Nortje}

Master Thesis by Composition

Victoria University of Wellington

School of Design, Media Design 


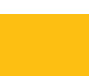




\title{
PERSISTENCE OF ILLUSION
}

\section{Using spatial illusion as a visual performance mechanism}

\author{
by
}

$$
\text { Johann Nortje' }
$$

A thesis by composition

submitted to the Victoria University of Wellington in fulfilment of the requirements for the degree of

Master of Design

in Media Design

Victoria University of Wellington

2010 
Johann Nortje BDes Industrial Design (Hons) Victoria University of Wellington

Submitted to the Media Design Department School of Design

Victoria University of Wellington March 2010 


\section{Acknowledgements}

Having personally come from an Industrial Design background, my supervisors

Anne Niemetz and Douglas Easterly provided me with a real eye opener in terms of direction and exploration within Media Design, adding immense significance to my research. Their patience and dedication acted as great moral support throughout the various ups and downs of this thesis, which overall I sincerely thank them for.

Furthermore, Morgan Barnard's extensive scope of knowledge and experience proved invaluable to building my skill-base and I thank Morgan for sharing my excitement in resolving even the smallest of challenges.

Lastly, I would like to thank Tammy Thompson for her twist on the creative input and initial motivation towards the project, Melissa Cowan for her excellent photos and my fellow master students for their support through the long grinding hours. 


\section{Abstract}

This thesis presents the design of a real-time visual performance system for live performances. Building on a research analysis of historical context and precedents, it is evident that software systems currently available to Live Cinema and VJ performers are often complex to navigate and counter intuitive to perform with. An alternative approach to visual performance system design is investigated in this thesis, where the spatial zone of the physical performance is used as the basis for the design, rather than purely placing the focus on software architecture. The investigation focuses on how the creation of live visual content can be achieved through the virtual and physical spatial relationships within the performance and how the performer then interacts with these relationships through bodily response and navigation. This is achieved through combining the successes of contemporary visual performances, the interaction techniques used in pre-cinema instrumentation and the use of projection mapping as a means of visually addressing the entire space of the performance. These investigations are demonstrated through a series of experiments and theoretical studies culminating in a set of design criteria, put together in a final system design accompanied by a demonstrative performance. The significance of this research is to provide the design basis for a successfully intuitive visual performance instrument, which can provide immediate results yet still require skill and experience to master. This will move the skill base of visual performance away from software navigation and more towards the physical ability to create and perform complex visual compositions in real time. 


\section{Contents}

Abstract

Acknowledgements

Table of contents

1. Introduction

2. Context Analysis

- Synaesthesia and the study of Visual Music

- Visual Music Instruments

- Interaction

- Performance

- Projection Mapping

- Summary

3. Design Experiments

- Theremin

- Projection Mapping Experiments 1 - 3

- Summary

4. Research Analysis

- Towards a Synaesthetic Performance

- Moving Beyond the Screen

- Space Medium

- The Loop

- Instrument as a Metaphor

- Summary

5. Design Criteria

- Design Criteria for Visual Music Instrument

- Summary

6. Final System Design

- Overall System, Interaction and Interface

- Software Setup

- Performance Documentation

7. Conclusions

- Discussion

- Conclusion

APPENDIX 1

APPENDIX 2 


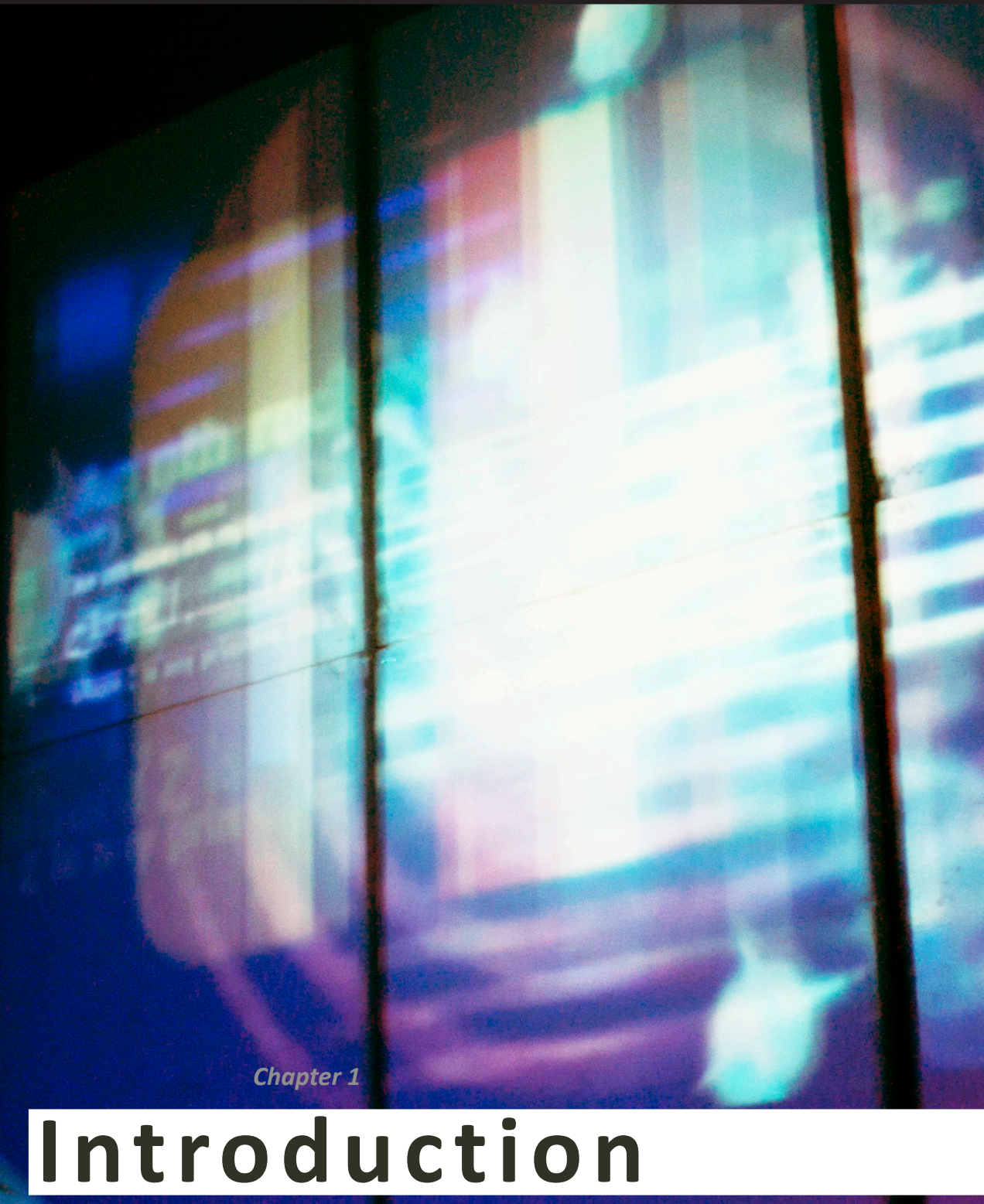




\section{Introduction}

\section{Motivation}

This thesis will explore the theoretical framework for the design of a visual performance system. These systems generally exist as software applications for VJ's and Live Cinema Artists. My personal interests and expertise as a VJ lie in this field and I have come to encounter a series of issues in regards to creating live visual performances. The visual content in such a VJ performance is often generated live, or cued live, in response to the music in the environment, yet as the VJ artist is often stuck behind a laptop computer, his influence can go by quite unnoticed. The visual performer (VJ) is not necessarily seen as a performer due to the lack of what could be called a 'performer presence,' caused by the intense focus on software navigation by the VJ not visible to an audience.

Most software available to the visual performer is often complex and counter intuitive to interact with. The skill seems to lie with how apt the performer is at navigating through the software, rather than his ability to create and perform a visual composition. This is not a reflection on the artist, but more a critique on the tools not being able to provide the artist with the necessary support to perform freely and intuitively. Intuitive performances have been explored in a variety of fields in new media and by learning from these examples, the area of VJ performance can definitely benefit.

\section{Objective}

An ideal visual performance system would allow for a more intuitive approach to creating visual performances, with the focus of interaction placed on creating a performer presence. It is the objective of this thesis to establish a framework based on the artist's navigation of the performance space, in order to design a fluid, organic and spontaneous system of interaction. The aim is to combine computer sophistication in output of visual compositions with the interaction techniques established by pre-cinema instrumentation. This combination will be applied to the technique of projection mapping, using the entire space of the performance as the visual content generator and to provide the guidelines for interaction. Spatial notions of interaction can create a successful visual performance instrument that is easy to understand, easy to navigate; yet complex in possibilities of performance interaction and visual content generation.
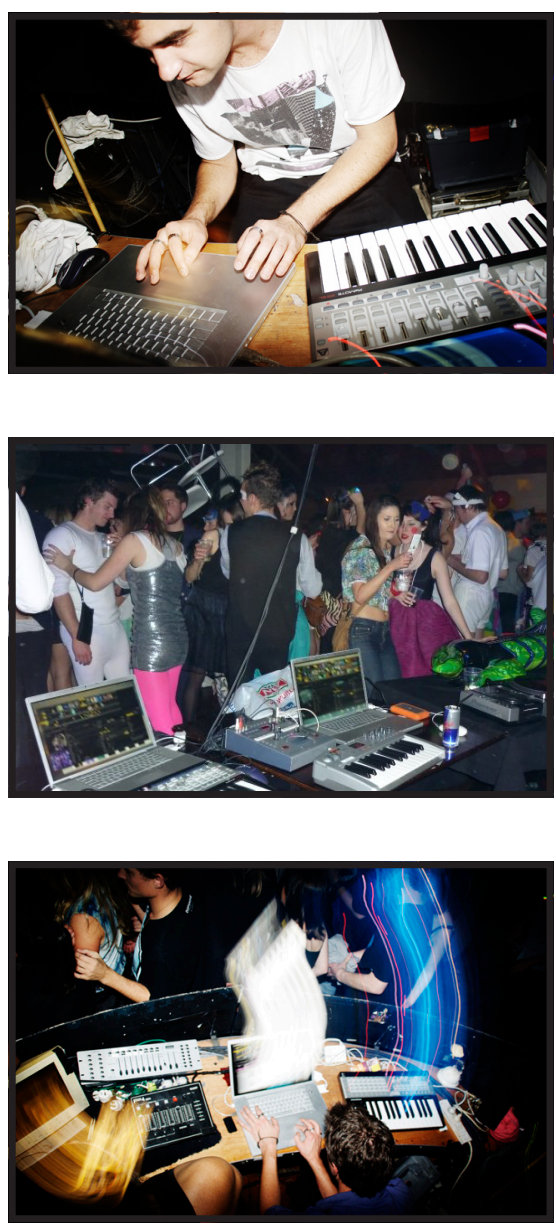

Figure 1 - 3

Examples of VJ set-ups 


\section{Overview}

This thesis, alongside the compositional element, aims to establish a framework from which a visual performance instrument can be designed. The research is focused around using spatial visual illusions as grounds for generating a visual performance. This places the performer in the role of the 'conducting illusionist,' providing the basis for designing the visual performance and interaction. The artist can now be a major component within the performance by placing him within this metaphorical concept of the illusionist. This concept then moves the visual artist away from being an unnoticed contributor and into a vital part within the performance.

It seems contemporary systems for visual performance has taken a step backwards form the more intuitive interaction techniques used in pre-cinema instrumentation. Spatial consideration in terms of visual content has also merely gained attention within installations and not so much in live performances. The research therefore starts by asking: how can the creation of visual content be achieved through the virtual and physical relationships within the performance and how will the performer then interact with these relationships? The possibility therefore lies within this question to research and develop a visual performance system, by using the space within which the performance exists to drive content creation and interaction guidelines. Throughout the research, the term instrument is used to describe the visual performance system. The use of instrument as terminology covers the principles needed for a successfully intuitive system; ideals of simplicity in use yet remaining complex and illusive in possibility.

Initially the research is set out to determine the main aspects within the fields of visual-music, visual-music instrumentation and three areas of contemporary performance; interaction, performance methodology and projection mapping. These aspects are then tested through a series of experiments to investigate all the principles and properties identified in the research and to gain a practical understanding of the underlying systems. A thorough research analysis follows, forming a theoretical angle towards visual performance systems, aimed at bringing together the entire process. 
design. The criteria are laid out as a set of aims and guidelines to outline the desired design solution for a visual performance system. Finally the system design is presented, documented and analysed as an illusionistic spatial performance.

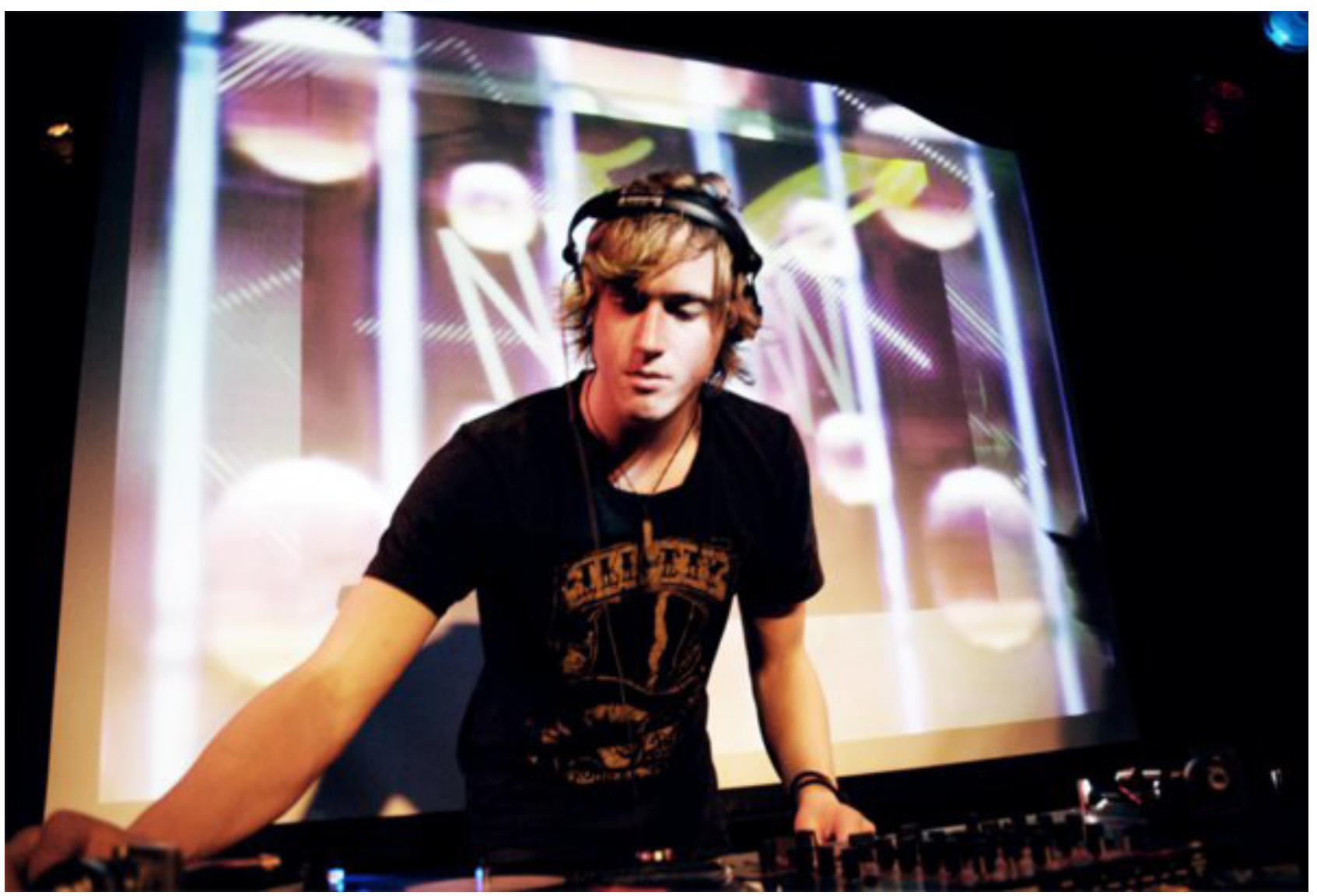

Figure 4

VJ display with DJ Realtime in front - demonstrating relevance of visual performance during an event 


\section{Synaesthesia and the study of Visual-Music}

The concept of synaesthesia is defined as a neurologically based phenomena where sensory perception of one kind manifests as a sensory perception of another, such as associating a certain colour with a certain musical tone (Mattis, Brougher, Strick, Wiseman, \& Zilczer, 2005). This is not to be misunderstood as both sensory perceptions being externally stimulated the same time but rather where the viewer experiences a stimulant through one sense and a secondary sense is associated. In effect the viewer becomes the synaesthetic medium. In the artistic search for abstraction, synaesthesia proves a viable source for inspiration. Synaesthetic sense perception can be broken down into discreet units (Mattis et al., 2005) where each unit is transposed into a different sense. In this instance we can use the elements of music and analyse, as a theoretical study, how they would translate visually. In music these units such as notes, harmonies and compositions would create a perceptive sensation finding it's equivalent in visual mediums such as colours and shapes (Mattis et al., 2005). In effect these visual elements can then be combined to form a visual composition much like a musical performance piece. For introduction this can be described as 'Visual-Music'

Early Twentieth Century artists, such as Wassily Kadinsky, Paul Klee and Frantisek Kupka, attempted to capture this relationship in their paintings, paying attention to the fluidity and colour-scapes through which music can be interpreted. These specific attempts remain mainly static, two-dimensional representations, and can only offer a glimpse into a truly synaesthetic experience. Visual-music however, has been investigated long before through instrumental systems that could generate and perform visual-music compositions. The earliest known device to perform colour-music was the 'Ocular Harpsichord' built by Father LouisBertrand Castel in 1734. Later attempts were Thomas Wilfred's 'Clavilux' in 1919, for example, which was built to demonstrate spiritual principles or 'Lumia,' and Oskar Fischinger's Lumigraph, built in the 1940s, that allowed one to 'play light.' These instruments were designed for use in solo performance or in combination with music, with the aim to generate a new aesthetic experience: the 'Synaesthetic Cinema'. Gene Youngblood wrote in his book Expanded Cinema: 'The fundamental subject of synaesthetic cinema - forces and energies - cannot be photographed. It's not what we're seeing as much as the process and effect of seeing: that is the phenomena of experience itself, which exists only in the viewer (Youngblood 1970). Synaesthetic Cinema therefore endeavours to create an 
Isaac Newton

Louis Bertrand Castel

George Field

D. D. Jameson

Theodor Seemann

A. Wallace Rimington

Bainbridge Bishop

H. von Helmholtz

Alexander Scriabin

Adrian Bernard Klein

August Aeppli

I. J. Belmont

Steve Zieverink

Figure 5

Artists and scientists interpretation of colour mapped to a musical scale

1704

1734

1816

1844

1881

1893

1893

1910

1911

1930

1940

1944

2004
Three Centuries of Colour Scales

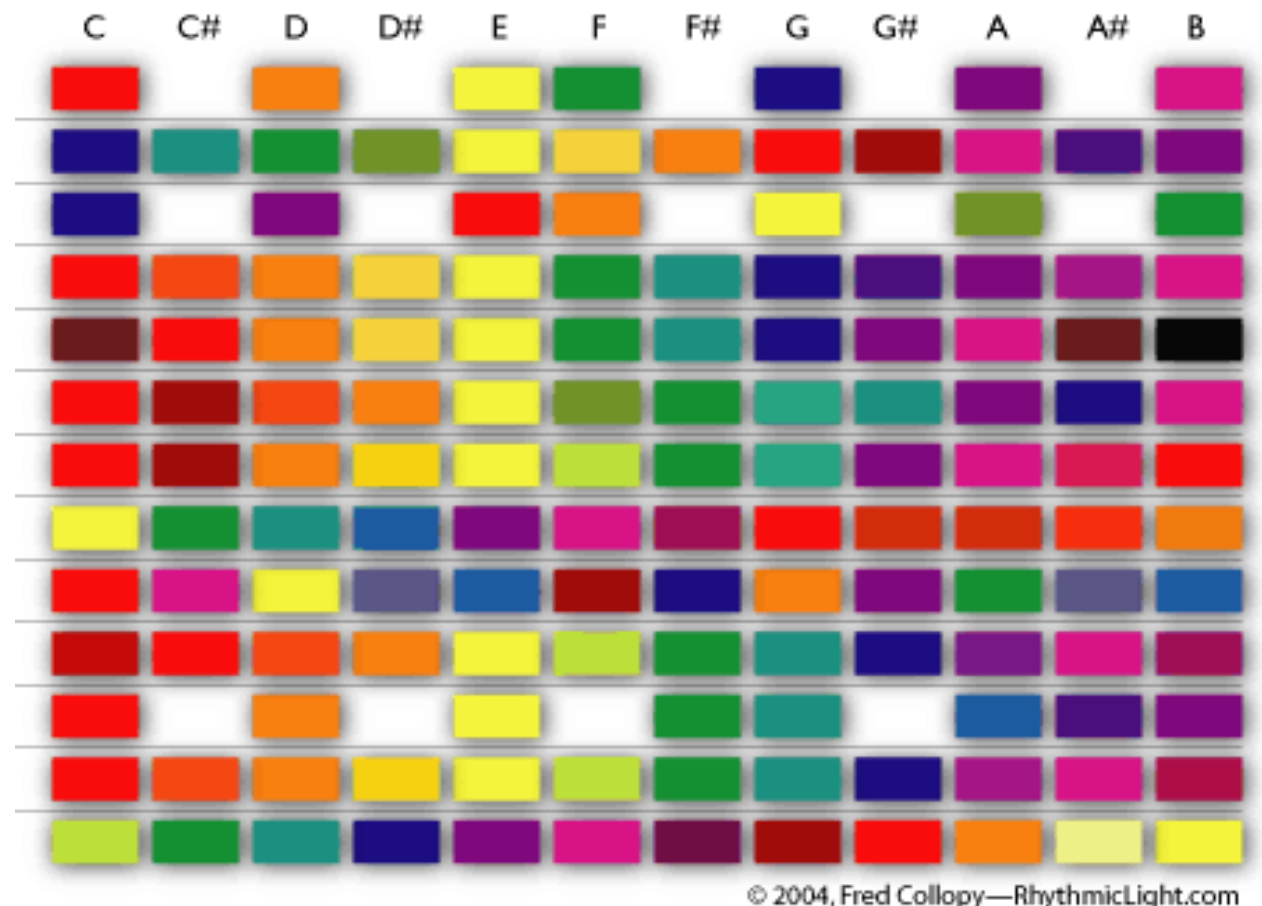

experience within the audience through an abstract visual composition, to stand equally alongside a musical performance.

Synaesthesia can be considered a very complex art form as it utilizes the viewer to complete the interpretive process. The symbolist movement in particular viewed synaesthesia as a 'mystical vehicle to attain a higher reality or state of consciousness' (Mattis et al., 2005). As the viewer is the final piece in the puzzle, the synaesthetic experience is individual to each person. Arthur Dove speaks about this experience in saying that 'perhaps art is just taking out what you don't like and putting in what you do. There is no such thing as abstraction. It is extraction...' (Dove 1929). This process of abstraction created within synaesthesia, or breaking down into discreet units, is a personal interpretation, a personal extraction of certain units. These transitions of synaesthesia are completely tied to the thought process of the viewer. So as a viewer can come to understand the emotional intent of a musician through a musical piece, so should the viewer be able to see the emotional intent of the composer through a visual-music piece. Colours, like music, are sensory stimuli that evoke this 
emotional response (Mattis et al., 2005).

The term visual-music that was first used by Roger Fry when describing art that 'give up all resemblance of natural form, and create a purely abstract language of form - a visual music' (Mattis et al., 2005. Cited, 25). This form of art has been investigated since the start of the $20^{\text {th }}$ Century as a compelling ideology. In 1900 Louis Favre called for an art of colours in motion, which became an investigation into variation of colour and composition as an interpretation of musical progression, the focus for many artists investigating such combinations as interpretations. There exists this search to explore the fluidity and rhythm of music avoiding the use of geometric forms, and even if they are used, they are combined in such a way as to explore a more fluid combination of elements. Individual analogies of parts within the whole can be identified, and colour wise there is evidence that the artists were trying to recreate the emotive qualities that the music offers.

Many attempts have been made to analyse specifically how colour represents music. Again we are brought into the breakdown of elements in finding this correlation. Scientists and artists alike have investigated the 'colour of sound' and more often they are related to the pitch register of the sound, where lower pitches are represented as reds moving up in the pitch range through yellows, greens, blues and purples (see figure 5). This strangely, yet almost un-coincidently, is almost a direct correlation to the colour spectrum. Whether coincidence or mathematical it is intriguing to note that such correlations can be made. In 1914 Maud Miles made a comment on this form of art in terms of representation. 'The truest parallel that I can conceive between direct light rays of colour and music would be to lay aside all attempts to represent objects either in a natural or conventional way, in using colour. To simply use colour as music might prove a genuinely new art' (IRSA 1987). In the later part of the $20^{\text {th }}$ Century technology had advanced and artists such as Len Lye were able to represent visual music as a moving composition no longer constraint by a static canvas. This gave way to the possibility to express time and rhythm through a composition. Still the idea was to avoid conventional geometric shapes and let the rhythm and composition of the sounds guide the visual creation, thereby generating forms through the composition of the fluidity created by these colour scapes.
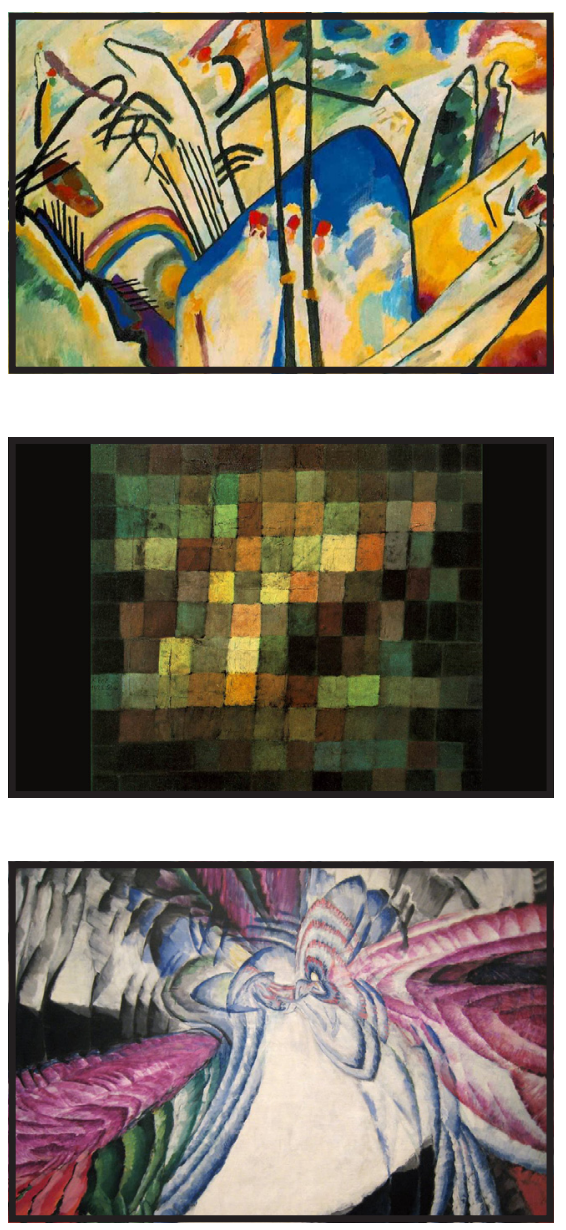

Figure 6

Wassily Kadinsky 1911

Composition IV

Figure 7

Paul Klee 1925

Alter Klang

Figure 8

Frantisek Kupka 1913

Organization of Graphic Motifs II 

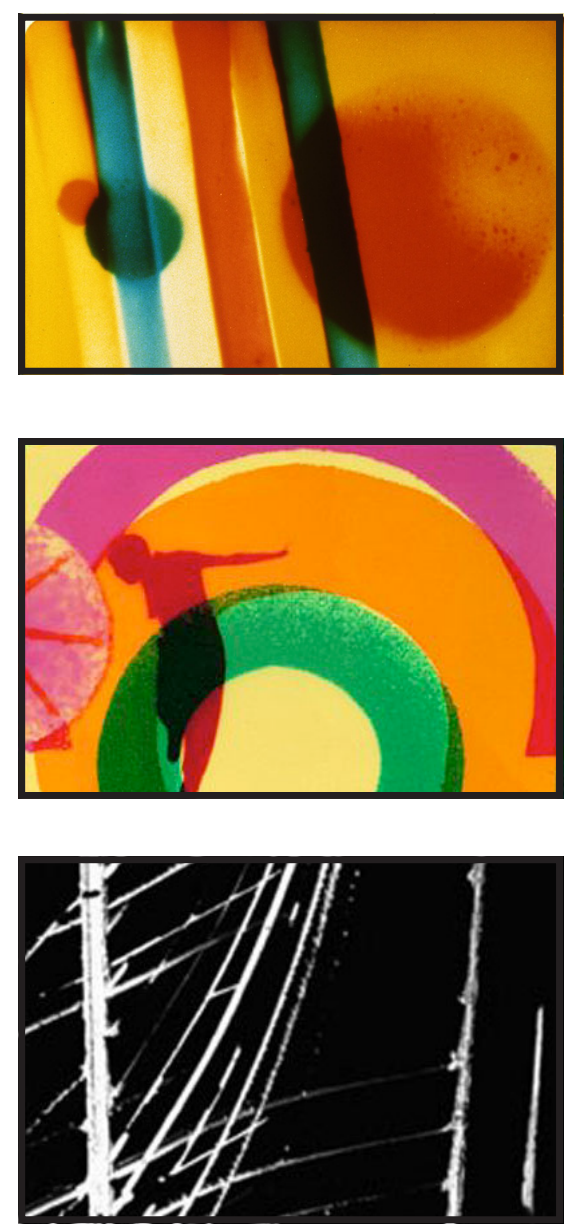

Figure 9

Len Lye 1938 Colour Flight Stll

Figure 10 Len Lye

Rainbow Dance Still

Figure 11

Len Lye 1958
On the other hand there is the theory that visual music should be evaluated on its own basis separated from music. Jean-Jacques Rousseau commented on this topic;

I have seen the famous harpsichord on which music may supposedly be made with colors. Not to recognize that the effect of colors is in the permanence while that of sound is in their succession is to be unaware of the workings of nature... Colors are the attributes of inanimate things; all matter is colored. But sounds announce movement... Each sense, then, has its proper field. The field of sound is time, that of sight is space. To multiply sounds heard at one time, or to develop colors one after the other, is to change their economy (Morgenstern 1956).

There is an important note that can be taken which places the emphasis on the distinction between time and space. The time and space distinction Rousseau makes refers to the compositional structure of both sound and image, but is in fact a very simplified explanation. The real relationship is much more complicated, as although a musical composition is based over time it cannot exist without space. In the same manner, although a moving visual composition is based in space, it cannot exist without time. Returning to synaesthesia as the breakdown into separate units and these transposed into a different sense, we can start to see the need for a relationship that can be drawn between time and space in the synaesthetic experience. As music becomes a composition through time that cannot exist without space, so we can start to evaluate visual-music as a composition through space that cannot exist without time. Thomas Wilfred does warn that 'Lumia may never be played in the same manner as music... attempts to design Lumia instruments in imitation of musical ones will prove as futile as attempts to write Lumia compositions... by following... rules... for music' (Wilfred 1948). This places visual-music in a performative category of it's own, a performance through space, one much closer to a musical performance than the initial attempts of canvas paintings.

However, the idea of synaesthetic performance remained largely untapped by mainstream cinema. The opportunity to create a cinematic performance in realtime was not pursued; instead, the moving image was developed towards the 
Narrative Cinema. Nonetheless a movement developed during the 1960s and 70s took upon the notion to move the cinematic experience beyond the narrative, and to generate a more abstract performance-orientated intervention, returning to the concept of the synaesthetic experience. This movement for the so-called 'Expanded Cinema' advocates a cinematic experience that is created, composed and performed live, utilizing the flow and progression of the music to create a visual performance and is therefore based on intuitive decisions and responses, culminating a lot of the ideas set out by the visual-music ideology. 


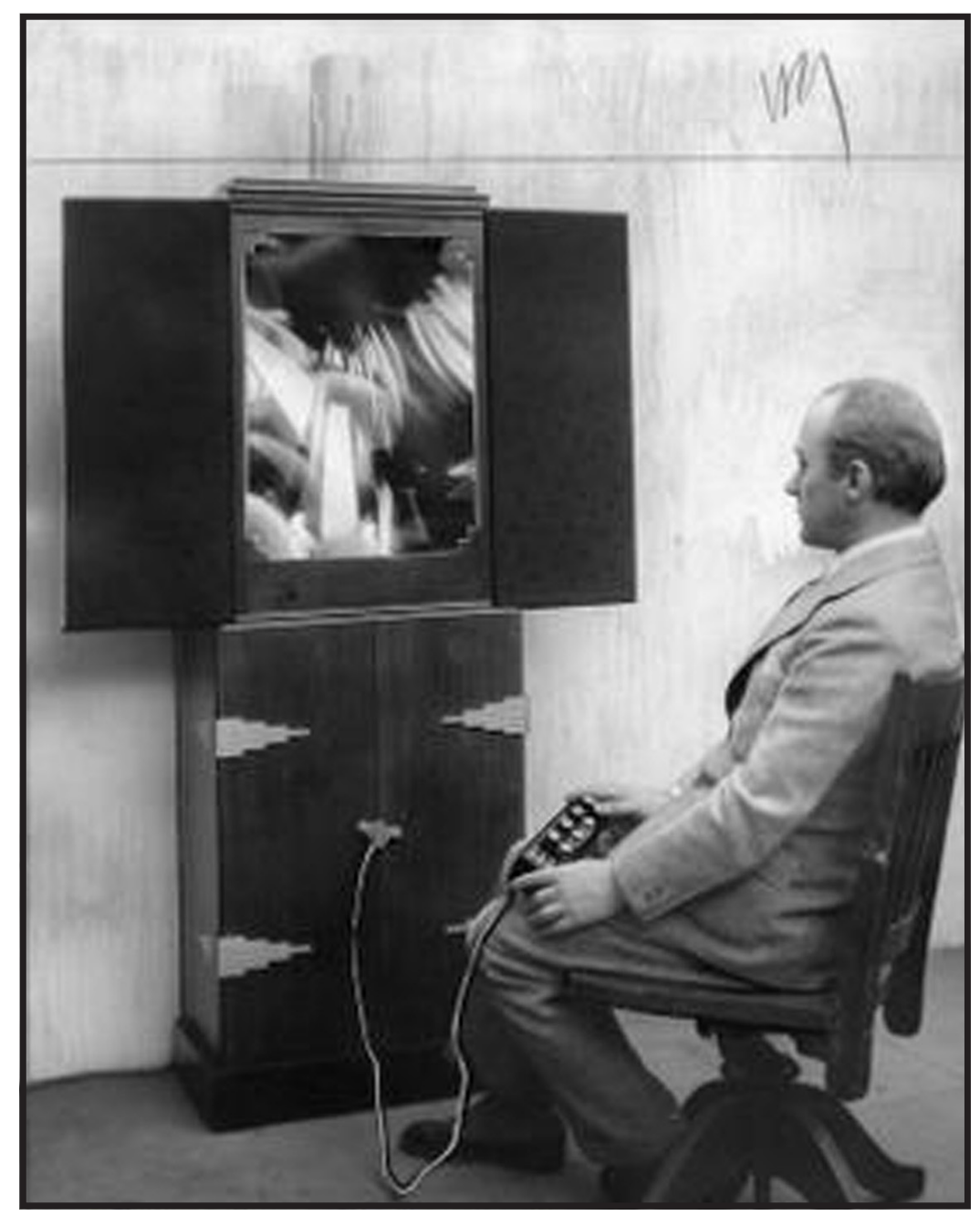

Figure 12

Thomas Wilfred performing with Clavilux Jr Home Edition 


\section{Visual Music Instruments}

\section{Pre-Cinema Instrumentation}

In the case of Pre-Cinema Instrumentation quite a few developments and experiments were made, however in this case the discussion will centre around the Clavilux of Thomas Wilfred and the Lumigraph of Oscar Fischinger, as they belong to the most influential works in the field of visual-music instrumentation. A more general and all encompassing visual timeline is provided in Appendix B.

\section{Clavilux - Thomas Wilfred - (1889-1968)}

Thomas Wilfred joined the Theosophist Society when he moved to America who wanted to create a colour organ that would demonstrate spiritual principles. Initially the investigation set out to establish a relationship between colours and sound, however Wilfred moved on from this to create an art of pure light. The Clavilux was designed to perform what Wilfred referred to as 'Lumia.' This visualized as streams of fluid coloured light morphing together on screen. Wilfred argues that Lumia should not be governed by the rules of musical composition, or be attributed principles that apply to static composition and colour harmony.

The Clavilux consisted of a large keyboard with sliding keys, several light projectors and reflectors, with the design resembling very closely the musical organ with its pipes (Popper 1968). The visual Lumia was controlled indirectly through an interface, with no interpretation of natural human bodily movement. The interface is a remote control, with dials and switches that is used to change and control the movement and colours of the visual imagery. Wilfred developed a variety of sizes from a 32 projector public performance instrument, to 'Lumia Boxes' which were self contained units able to play for days on end without visual repetition, through to 'Home Clavilux Systems' which much like the television were for personal visual performances (Moritz 1997).

\section{Lumigraph - Oscar Fischinger}

Animator Oscar Fischinger developed the Lumigraph; an instrument that allows one to play light (Moritz 1997). It is evident through Fischinger's performance of this instrument that he made a deliberate decision for the visual performance to accompany a musical composition, as the user interaction flows organically with the response to a musical piece. The design is two black boxes on either side to a latex screen contained in a frame. Inside the boxes are sets of coloured gels that are attached to a rotating wheel, projecting coloured light through a
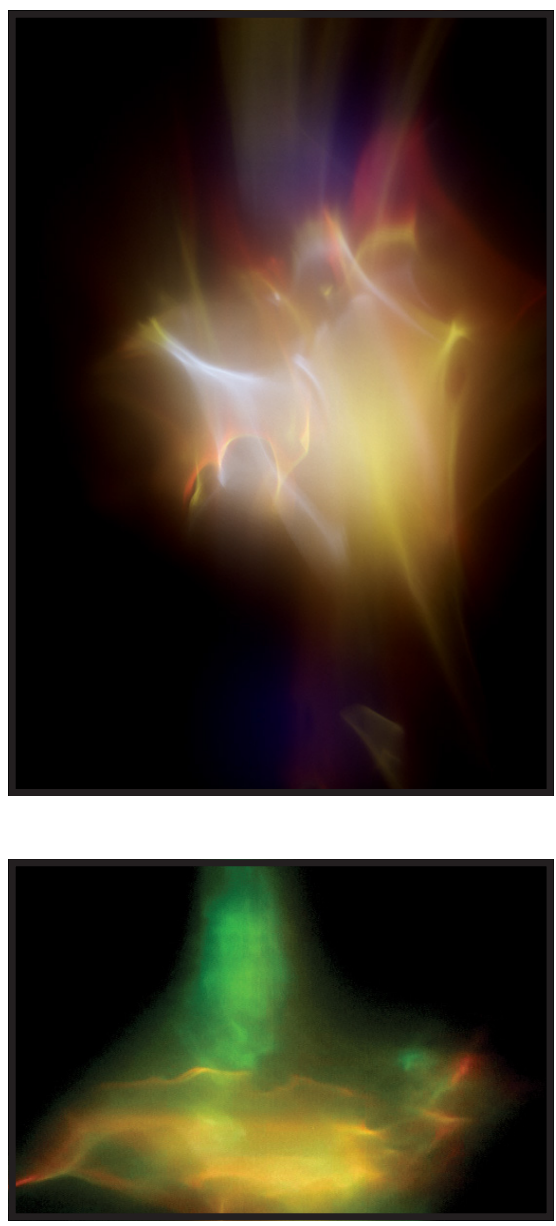

Figure 13

Clavilux Jr 91

Figure 14

Clavilix Opus 161

"untitled" 

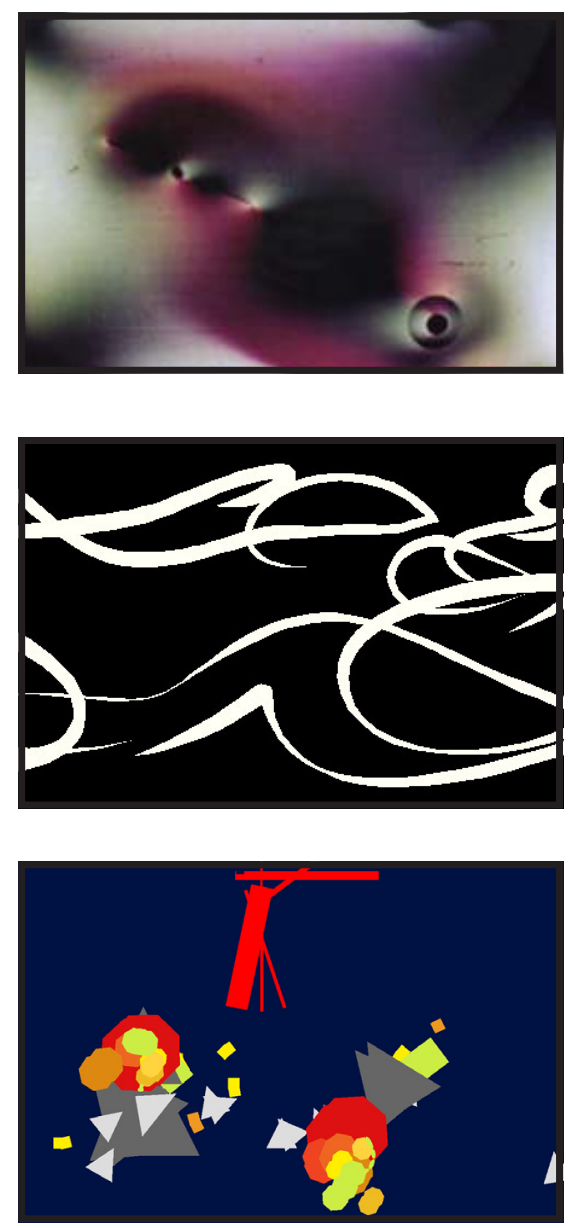

Figure 15

Oscar Fischinger Lumigraph still

Figure 16 Golan Levin Yellow tail still

Figure 17 Scott Snibbe slit in the box. The thin layer of light is cast just in front of the latex screen where any interaction with the screen would move it to intersect with the stream of light, instantly revealing the trace of the interaction. Two people would generally operate the Lumigraph, one on the screen performing the composition and the other controlling the colour wheel.

The creation of the visual image is a direct relationship between the body of the user (in this case the hands), which directly control the ephemeral marks left from the interaction with the screen.

\section{Contemporary Instruments}

\section{Mark Making}

Media artist Golan Levin approaches his thesis design into audiovisual performance systems based on the metaphor of painterly interfaces, where he asks whether the interfaces for visual performance systems need to be grounded in the act of drawing (Levin 2000). His main focus lies on creating an inexhaustible performance system, where the metaphor of digital 'painting' lends itself perfectly to such a task.

On the other hand, artist Scott Snibbe developed a series of interactive visual performance applications for pure visual communication, allowing the capture of spontaneous human gestures (Snibbe 1996). The applets developed, especially Motion Phone, seem to draw visual inspiration from abstract film animation. These applets are graphic systems that augment human movement based on the concept of painting, and recording these abstract painting animations enters them into a loop that becomes the visual performance.

John Maeda's Timepaint is a study of how virtual 'ink' could be used to perform and display dynamic computations, very much related to the art of drawing (Levin 2000). These drawings are created as temporal visualisations where the virtual 'ink' responds to real time conditions received from the time of composition, spatial attributes and user inputs. The significance of this piece is that the 'ink' can be temporal in nature, reactive and intelligent in behaviour towards inputs, creating ever-changing unlimited compositions. Maeda comments that "( $t$ )imepaint illustrates not just the lapse of a single frame of time, but the continuum of time in which the computer and user coexist." (Maeda 1995) 


\section{System Design}

Levin identifies three areas for research within Visual performance systems; the visual score, the interaction with control panels and the existence of interactive widgets. A score, as with a musical composition, burdens the mind with the cognitive load of translation and is therefore not an intuitive approach to visual performances. Secondly, Levin identifies that the expressive and responsive nature of analogue knobs has been lost through software replacements, and lastly he establishes that certain interactive widgets within software restricts user performance and are ultimately exhaustible, shallow or both

From his research, Levin identifies a set of desiderata that together define the properties of an ideal audiovisual performance instrument, which would yield unparalleled expressivity.

(Levin 2000)

- The system makes possible the creation and performance of dynamic imagery and sound, simultaneously, in real time.

- The system's results are inexhaustible and extremely variable, yet deeply plastic.

- The system's sonic and visual dimensions are commensurately malleable.

- The system eschews the incorporation, to the greatest extent possible, of the arbitrary conventions and idioms of established visual languages, and instead permits the performer to create or superimpose her own.

- The system's basic principles of operation are easy to deduce, while, at the same time, sophisticated expressions are possible and mastery is elusive. 


\section{Interaction}

David Rokeby evaluates Human Computer Interaction as an interactive feedback loop, one that exists with a parallel nature. Both sides in the interaction should be able to provide new responses and be able to react to given responses equally. Reflecting consequences of the user's actions and decisions back to him opens up the metaphor of a mirror, which in this case should not only reflect but also refract what is provided. The notion of direct control is now lost and becomes a relationship of encounter and involvement. This is aimed at establishing a close sense of relationship between the user and the experience (Rokeby 1996).

A large portion of Scott Snibbe's work focuses on creating temporal compositions, (Levin 2000) where the animation created becomes a part of the composition, constantly being added to. The aim of Snibbe's work is to provide an experience where the user can in a sense touch an immaterial world, 'with consistent and predictable reactions, but infinite variety.' (Snibbe 1998) As most of the work is based on computer vision as the interface, an indirect reflection of movement provided to the user acts as a visual dialogue. The actual graphic output of the performance becomes the visual component of the interface, essentially a feedback system available to the user. The performance is generally a result of user interaction indirectly connected to the visuals through control of exterior parameters such as filters and compositional elements.

\section{Computer-Human relationship}

David Rokeby identifies that using the computer as medium has a strong bias that must be worked against, when designing for interactivity. Rokeby (Rokeby 1986) evaluates this statement through the following relationships:

The computer language is purely logical, therefore the language of interaction should be intuitive

The computer is removed from the body, therefore the body should be strongly engaged when designing interactive works

Computer activity takes place on small integrated circuit, therefore the human encounter should take place on human scaled physical interaction The computer is objective and disinterested, therefore the interactive experience should be intimate 


\section{Input vs Output}

Media artist Golan Levin focuses very much on gesture capturing techniques when concerned with the input to a computer-human orientated system. He argues that the system of how the instrument works should provide the guidelines for how the physical input should take form. The gesture capturing techniques he refers to records and plays back a temporal component of a user's mark, which produces lively and organically animated results, creating a tight relationship between the user's input and the system's output. This, according to Levin, has important implications for the user, where they are directly guiding or directly controlling a display.

\section{Interface}

Rokeby identifies that the interactive interface has to encompass the entire zone of experience, a multidimensional encounter that is not focussed and definite. The language of the interface should be unclear initially, but reveal and evolve through exploration and experience. In comparison, Levin establishes that the interface should be quickly apprehensible and afford immediately satisfying results, yet at the same time provide a wide range of possible expressions to master over time. Both artists are masters of motion tracking and have used it extensively in their work, although in quite separate ways. Throughout his work Levin establishes the metaphor of a painterly interface, one where content can be painted, manipulated and deleted in a free form non-diagrammatic context (Levin 2000). This free form context exists with a visual mapping that eschews conventional mappings and instead relies on the user's perceptual system to intuitively interpret the system's rules. It is these rules that Levin focuses on in the design of the performance system, rather than the physical design of the interface. In both cases, Rokeby and Levin describe an interface that creates an intuitive experience between the system and the user, a contextual interface, unlimited by space or scale.
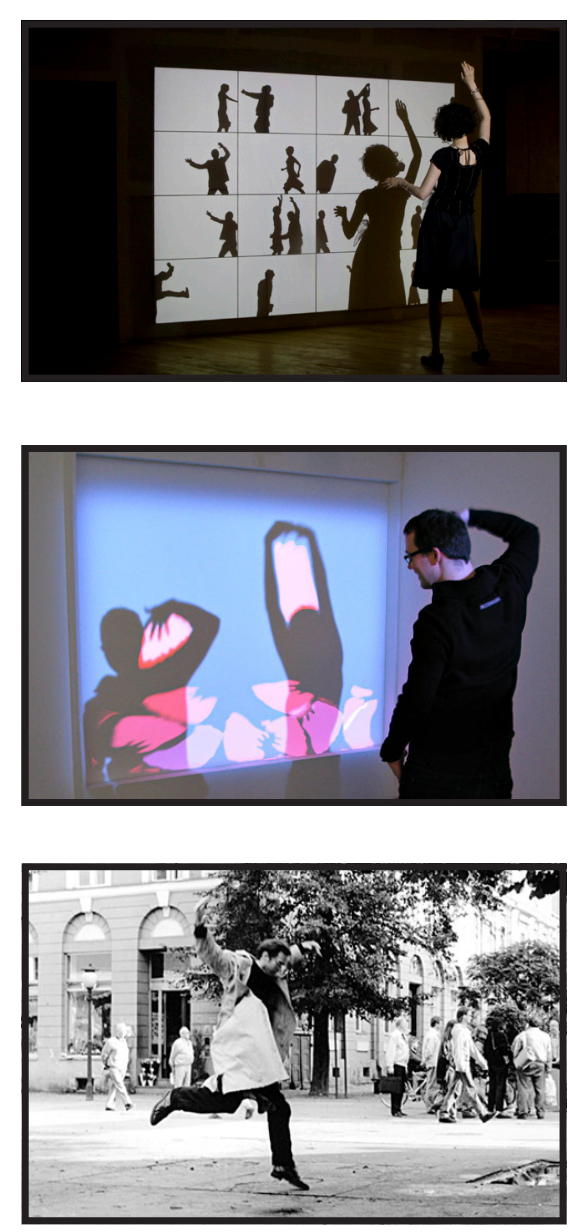

Figure 18

Scott Snibbe 2003

Deep Walls still

Figure 19

Golan Levin 2007

Interstitial Fragment Processor still

Figure 20

David Rokeby 1991

Very Nervous System 


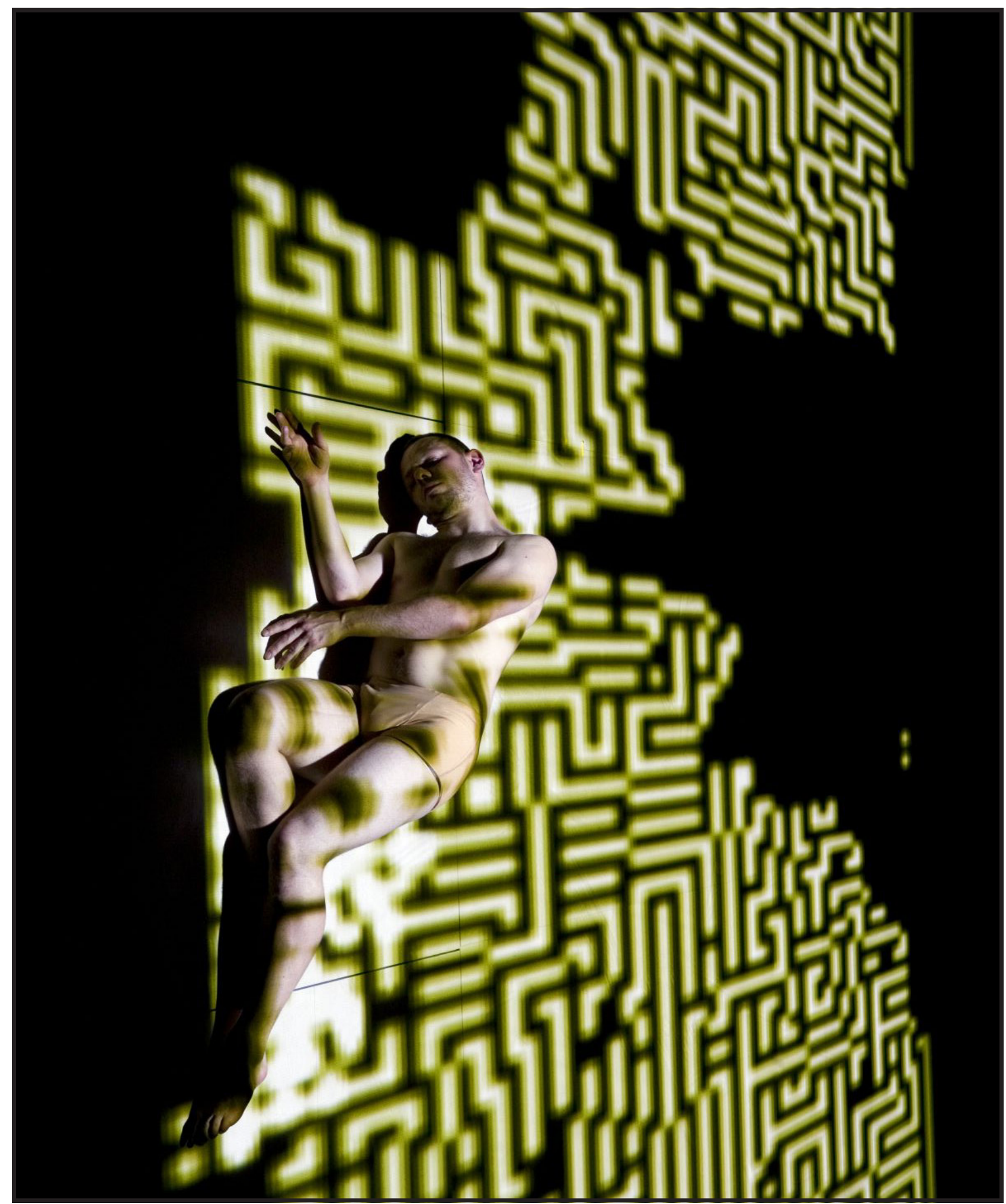

Figure 21

Frieder Weiss 2008

Mortal Engine still 


\section{Performance}

This section will discuss the performances as constructed by Media Artist Frieder Weiss and collaborators, comparing it to the guidelines put forward by Golan Levin in regards to a visual performance instrument as mentioned in the previous sections. Although Weiss's shows are stage performances, the analogy is made to demonstrate his performances as having a lot of similarities that potentially identify a visual performance system.

\section{Context}

Most of Frieder Weiss' visual performances are focussed on dance performances, where only the dancers are visible during the performance, and not the musician or video artists. The dancers on the stage become the active component, as they interface with the visual system through the movements of their movements, creating the visual imagery. Weiss uses camera tracking to follow the movements of the dancers, where he is able to track the subtle variations in movements from the dancers. The visual projections that become part of the performance are contextualised to the dancer, in so doing becoming a conversation between the dancer, the space and the visuals imagery. The visuals are generally projected onto and around the dancer, occupying the whole space on stage, but generating a focal point around the performers.

\section{Interactivity}

Computer Vision traces the movements of the dancers, which in turn cues the visual imagery that therefore responds fluidly to the bodily movements of the dancers. Weiss describes this interaction as an emerging dialogue between multiple facets of the dancers' identity. The interaction is based on a temporal and fragmented visual simultaneity through which the dancer can develop his actions (Weiss 2006). In comparison, Golan Levin notes that an ideal visual instrument would provide the performer with visual dimensions that are extremely malleable (Levin 2000). This malleability is evident in Weiss's work, where the visual imagery has to respond to quite spontaneous and often unrehearsed movements created by the dancer.

\section{Inexhaustibility}

For an instrument to be successful, Levin states that the results of that instrument should be inexhaustible and extremely variable (Levin 200). It must be able to afford many different kinds of compositions and must be sensitive to
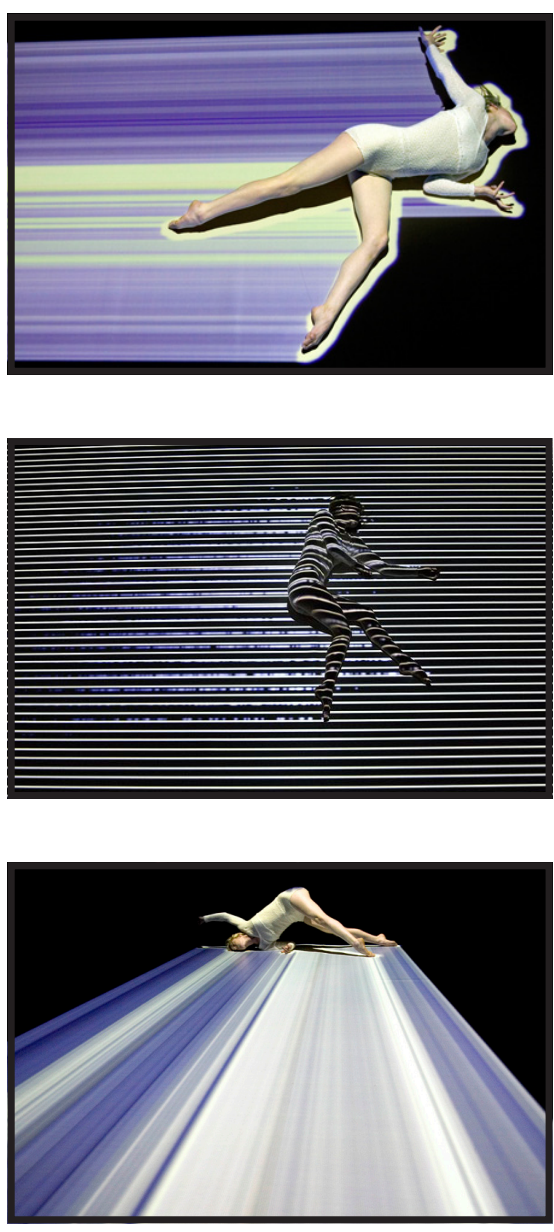

Figure 22

Frieder Weiss 2006

Glow still

Figure 23

Frieder Weiss 2006

Glow still

Figure 24

Frieder Weiss 2006

Glow still 
subtle features of a user's performance. These results, as variable as they might be, must be within the control of the performer, where the number of degrees of freedom must be matched closely to the number of expressive controls available to the user. Such subtle features described by Levin, translate directly to the movements of the dancer. The subtleties now provide the performer with an exceptional level of control and can therefore provide an extremely variable amount of results. Although the visual results can be somewhat predetermined, there will always be variations due to the subtle inputs received from the artist, as there are these subtle movement variations from the dancers in Weiss's work. The analogy of the dancer can now be used to describe a type of interaction, where if designed through these guidelines set forth by Levin, can provide inexhaustible results. 


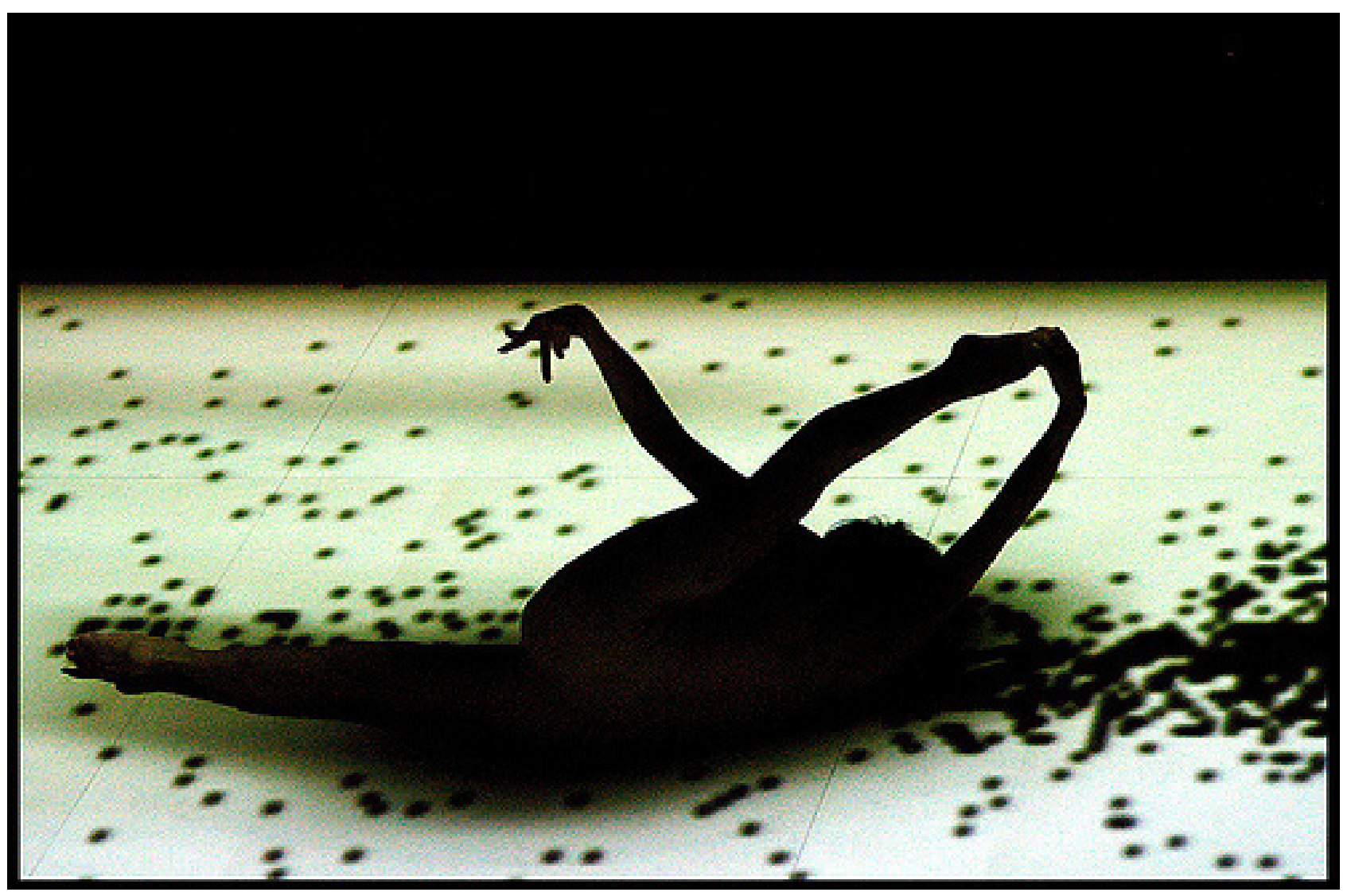

Figure 25

Frieder Weiss 2008

Mortal Engine still 


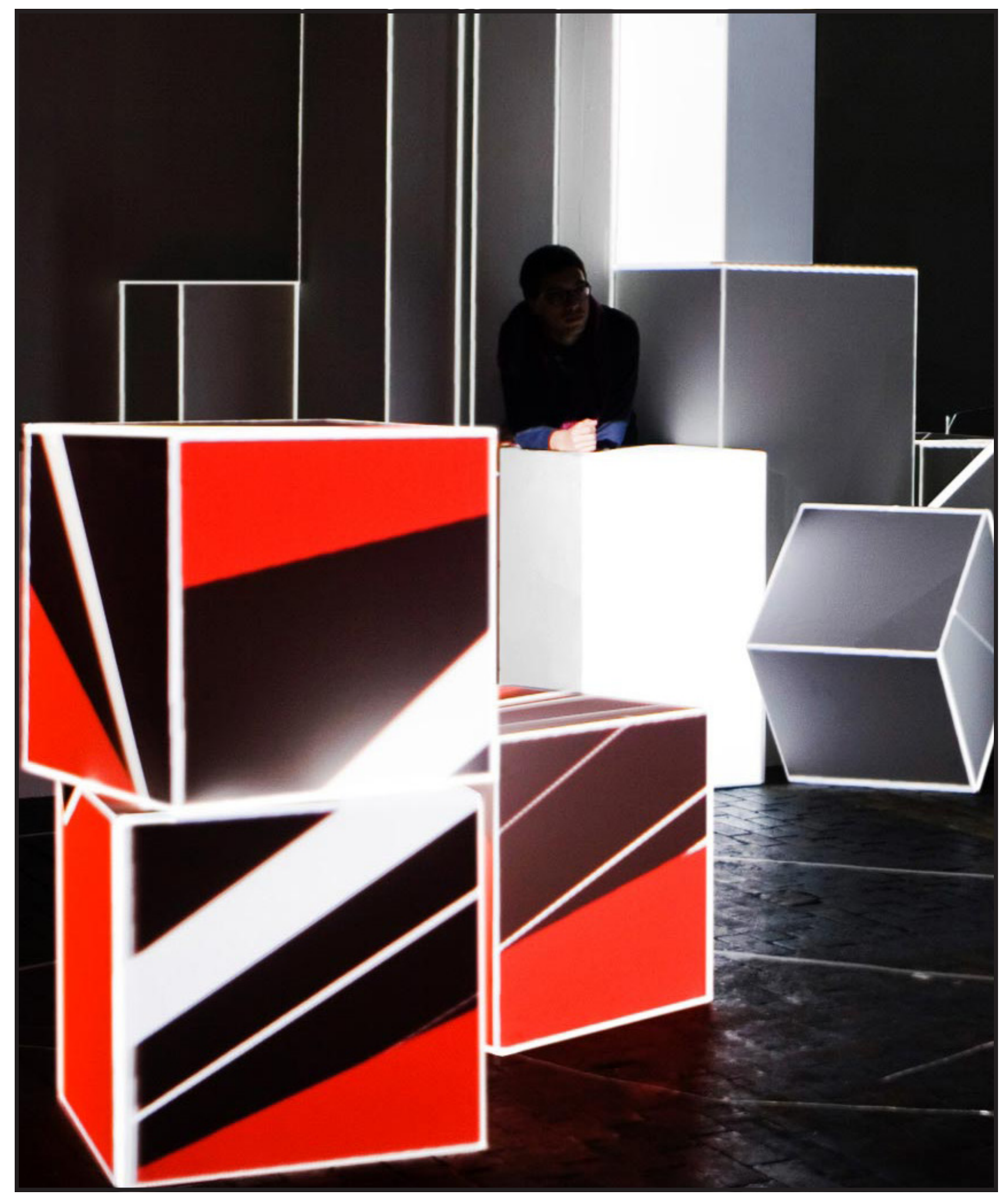

Figure 26

AntiVJ 2007 Mapping Festival

Cube Mapping Installation 


\section{Projection Mapping}

Projection Mapping, a form of augmented reality, is created when a virtual 3-dimensional scene is projected onto a matching physical 3-dimensional scene, matching the exact geometry and perspective. The illusion created, is that all the projected surfaces seem to be at correct proportions from any perpendicular point of view towards the surfaces. The visual imagery depends mainly on the physical scene and therefore Projection Mapping has the advantage of being quite a site-specific method. The site-specific character will allow every installation to have a very unique character. As Projecting Mapping is at this point still quite a new practice, this discussion will largely be based on examples to illustrate certain topics and points that are made.

\section{Virtual - Physical Relationship}

The aim of projection mapping is to replicate a physical scene within the virtual, modify it, and then project the modified scene back onto the physical scene as demonstrated in Figure 27. As the scene is projected back, each surface of the

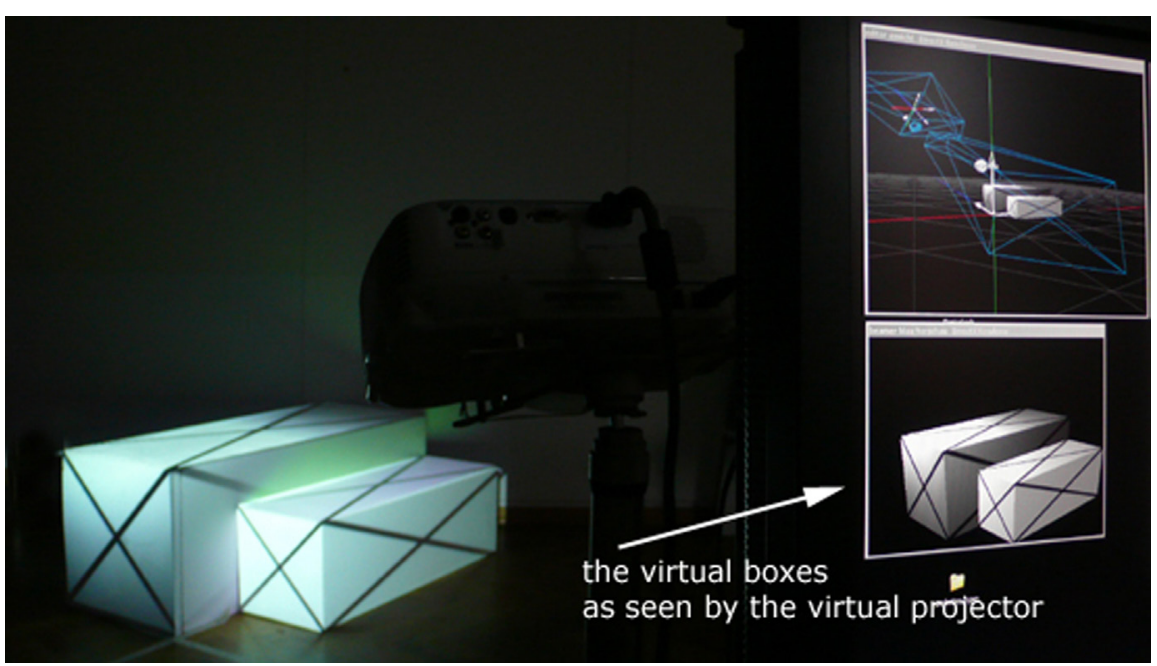

physical geometry becomes a screen in its own right. This provides the artist with the possibility to project images onto varying surfaces from only one projection source. As the projection boundaries are dictated by the actual physical geometry of the scene, the artist is able to create an optical illusion by altering the geometry of the virtual scene, in effect, illusively altering the physical scene as well. 
Figure 28

VVVV

'How to Project on 3D Geometry' Illustration of Perspective correction
In reality, as a person would move around a 3-Dimensional object, the perspective and visible surfaces would change. If the surfaces are purely mapped with graphical elements such as colours and borders, there is no issue in regards to perspective, however, once shadows and lighting angles are mapped, the viewer's point of view becomes very important. This is demonstrated through the example provided in Figure 28 and 29, where the viewer's angle is compensated for in the construction of the projected image.
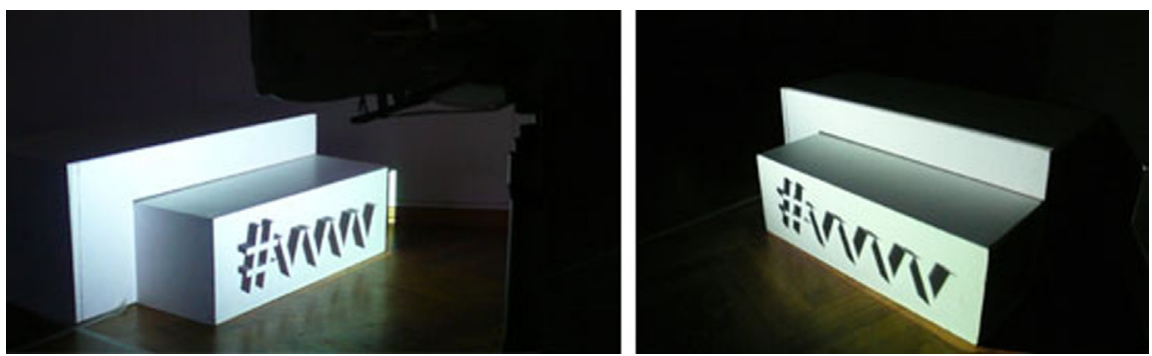

\section{Content}

This point of view correction is often seen as a useful tactic, as it provides the artist with the ability to create perceptive illusions. AntiVJ - A Visual Label (AntiVJ 2009), is one of the main protagonists in creating this spatial illusion. As described by themselves by introduction, their focus is on the use of projected light and its influences on the viewers' perception, creating illusions that challenge the audiences' senses (AntiVJ 2009). The perceptive illusion can now be a very useful technique in generating a visual performance. The actual content is very site specific, generated from the actual physical environment itself, providing a visual content beyond the purely graphical. The possibility also opens up for the artist to be quite involved in the performance much like that of an illusionist performer.

The content managed in projection mapping is not generally generated live, but consists of pre-recorded animations that can be triggered live by the artist. The content therefore tends towards a very narrative imagery. The most popular demand for projection mapping lies in building projections, where the actual geometry of the buildings are used to create narrative flow of graphical and filmic imagery. This field of visual performance is therefore still quite open in the areas of generating live abstract performance based content and interactive performability. 


\section{Summary}

This chapter analysed the background context within which this thesis is situated. It is evident from this analysis that visual-music should be evaluated on its own merits separate from such structures as put forth in music. The understanding is there that visual-music performance is a space-based composition performed through time. However by looking at contemporary visual-music systems it is evident that a reinvestment in the type of interactions used in pre-cinema instrumentation is needed. The possibility is open now to combine the language and effectiveness of visual-music compositions championed by media artists such as Golan Levin, and interaction techniques established by the pre-cinema instruments, with projection mapping installations as they provide a new paradigm for visual performances.

In the next chapter the discussion will contain experiments that I have carried out in relation to the contextual analysis, focusing on recreating and applying some of the principles mentioned in the research. The experiments will combine, and isolate certain features, to truly understand and discover the process of intuitive visual performance.
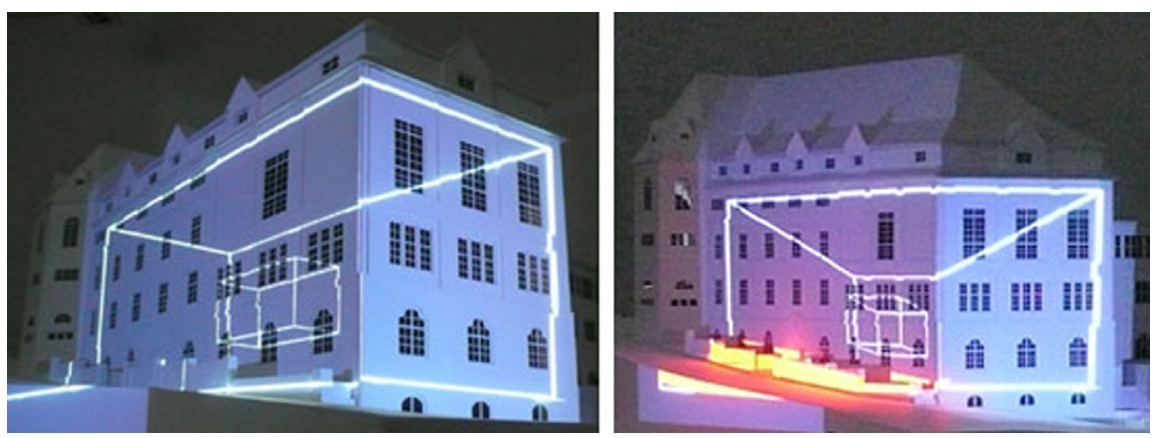

Figure 29

VVVV

'How to Project on 3D Geometry' Illustration of Perspective correction 


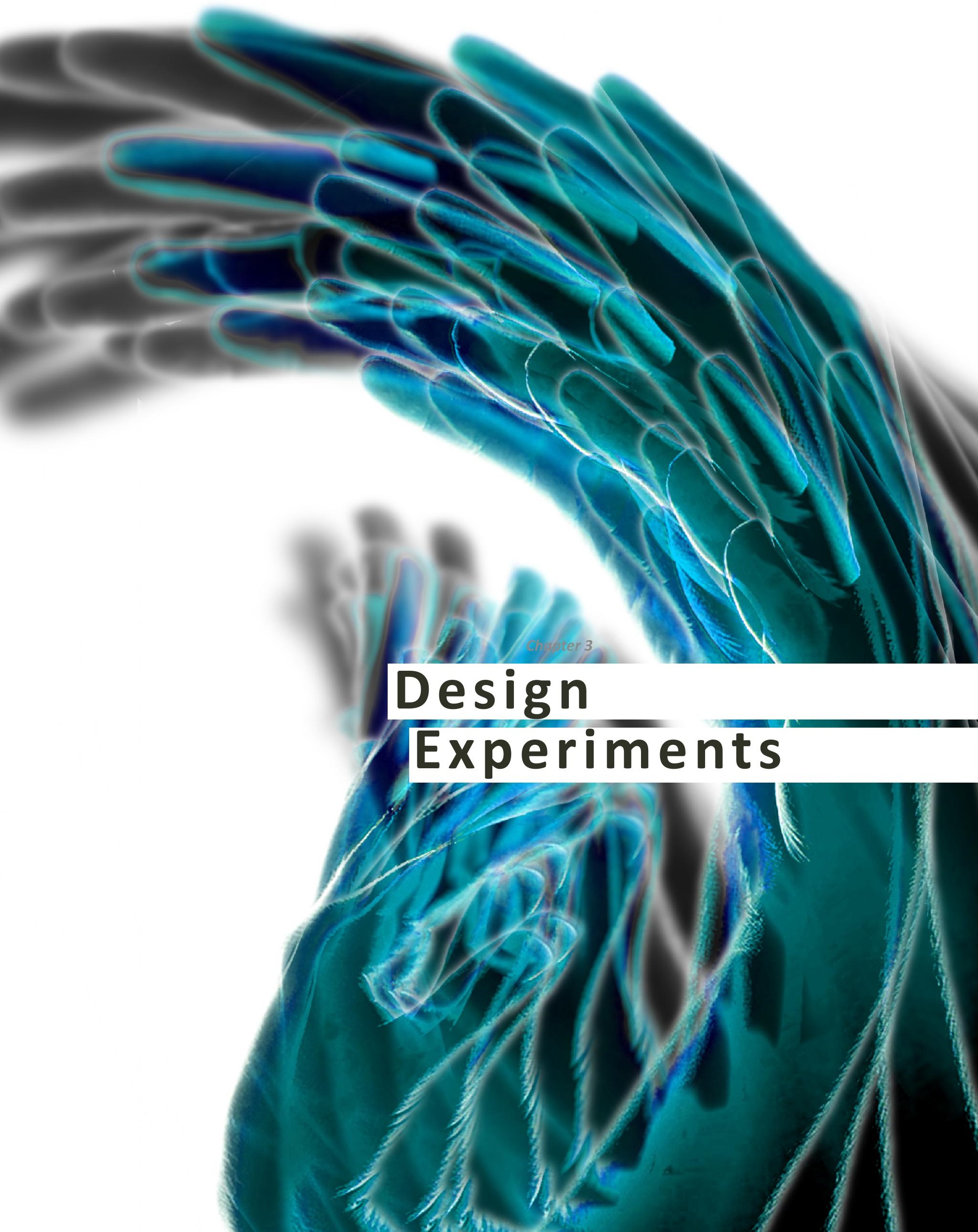




\section{Theremin}

The Theremin experiment was undertaken as a collaborative project with Tammy Thompson, a musician, to gain an understanding of controllable generated graphics through homage to pre-cinema visual music instruments. From the initial research into visual music, a few sketches were derived. These concepts were based on various layers of data and how they can possibly be interpreted as visual music. Looking at the specific interaction of the pre-cinema instrumentation such as the Clavilux and Lumigraph, it is evident that the performer mainly used two hands to generate the composition. As mentioned previously the Clavilux is controlled by values that are dialed in through a remote control, while the user placing their hands on the projection surface plays the Lumigraph. The Theremin instrument uses two antennae to detect the position of the performers hands generating varied electronic signals. In this respect the Theremin seemed the most appropriate as a starting interface. Using the principles established through pre-cinema visual music instruments, the methodology requires an interaction based on using both hands of the performer to create and manipulate the visual content.

The initial progression of the experiment was to generate colour architecture through the use of the two streams of data. Colour architecture in this case refers to the building, layering and composition of the fluid colour scapes. The information received from the pitch values of the Theremin, was mapped to a colour scale derived from the research on how colours are mapped to the musical scale (see Figure 5) and the volume data received was translated to the brightness of the composition. A very modest start, yet a good foundation and platform to build from. From here visual movement was introduced through the use of a prototype shape in the centre of the composition, which is controlled in shape and colour through the use of the pitch data. This initial composition can now be considered as the foundation and base expression of the instrument, or its "starting point."

These initial movements are just basic interpretations of two streams of data, where the most interesting work can now be introduced through the combination of these streams. Firstly, more elements are introduced that compliment the tonal range, providing a means to compose; secondly a set of filters can be applied to manipulate the composition. If the combination of the performers hands are thought of as an $x / y$ graph, the combinations of data
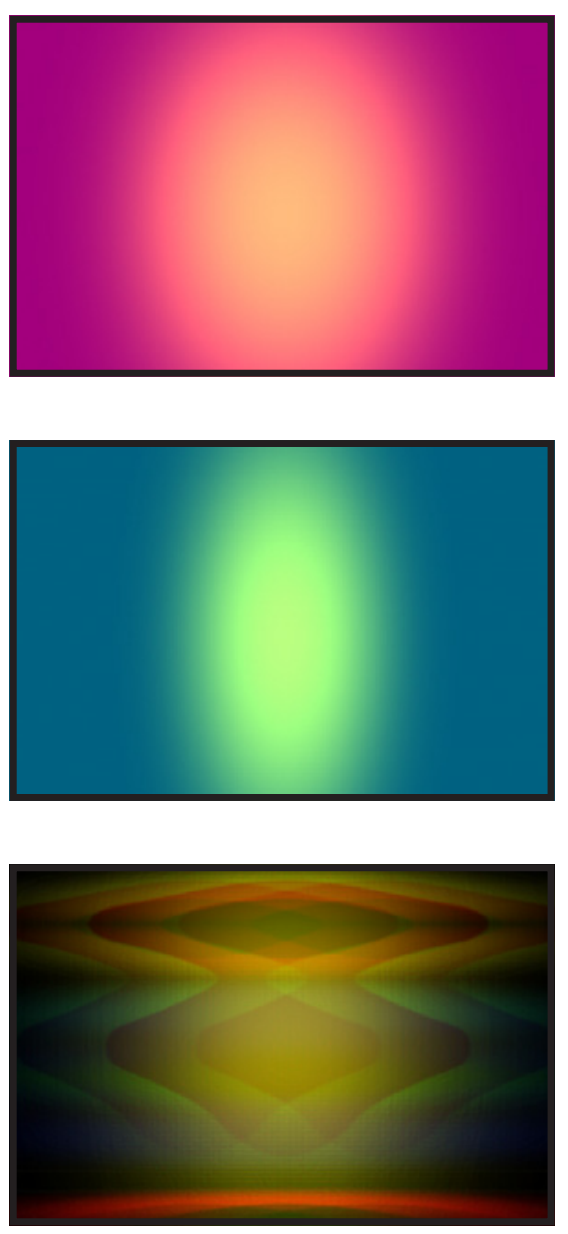

Figure 30

Starting point of the Theremin project display

Figure 31

Starting point of the Theremin project display with background colour changed

Figure 32

Theremin project display with two filters applied 
Figure 33

Graph indicating the various trigger pionts within the data that is provided by the theremin points can be set as certain "trigger points" that can activate situations within the composition. These trigger points are set up to be quite regular, so that an experienced performer can navigate to them quite easily, yet they are still quite discoverable to the novice user. This layering shows the build up of the composition, the intensity of the composer, and provides a fluid visual music instrument.

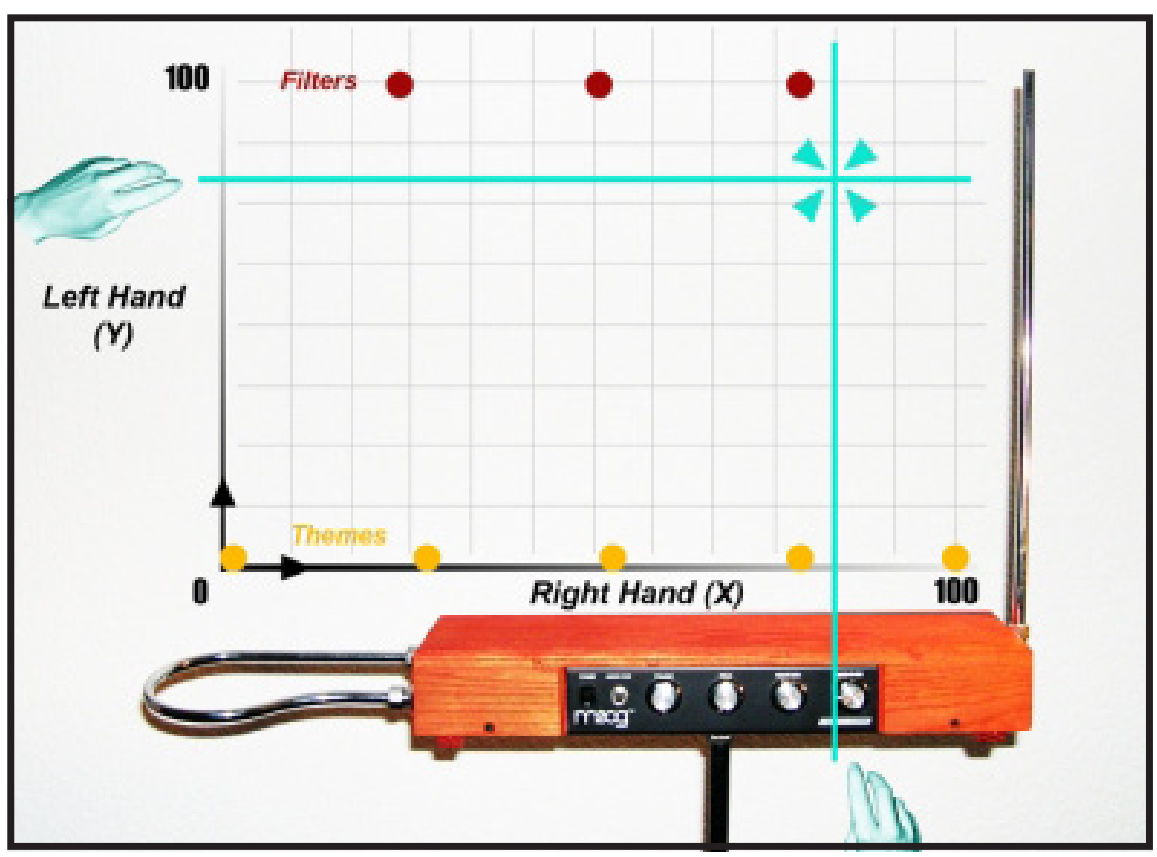

Each hand can play individually providing basic instantaneous results, however as the user becomes more experienced, they will learn the combinations of the two hands to produce much more fluid, unique and interesting results. From here there is a platform from which to build a visual music instrument to navigate through the colour-scapes. This template for gesture-orientated sense of immediate control provides a great basis for a sophisticated means of controlling generative visual content.

Figure 34 - 39 Various initial sketches that were tested during the build phase of the Theremin project. Note the introduction of various components to introduce movement and layerring into the composition 

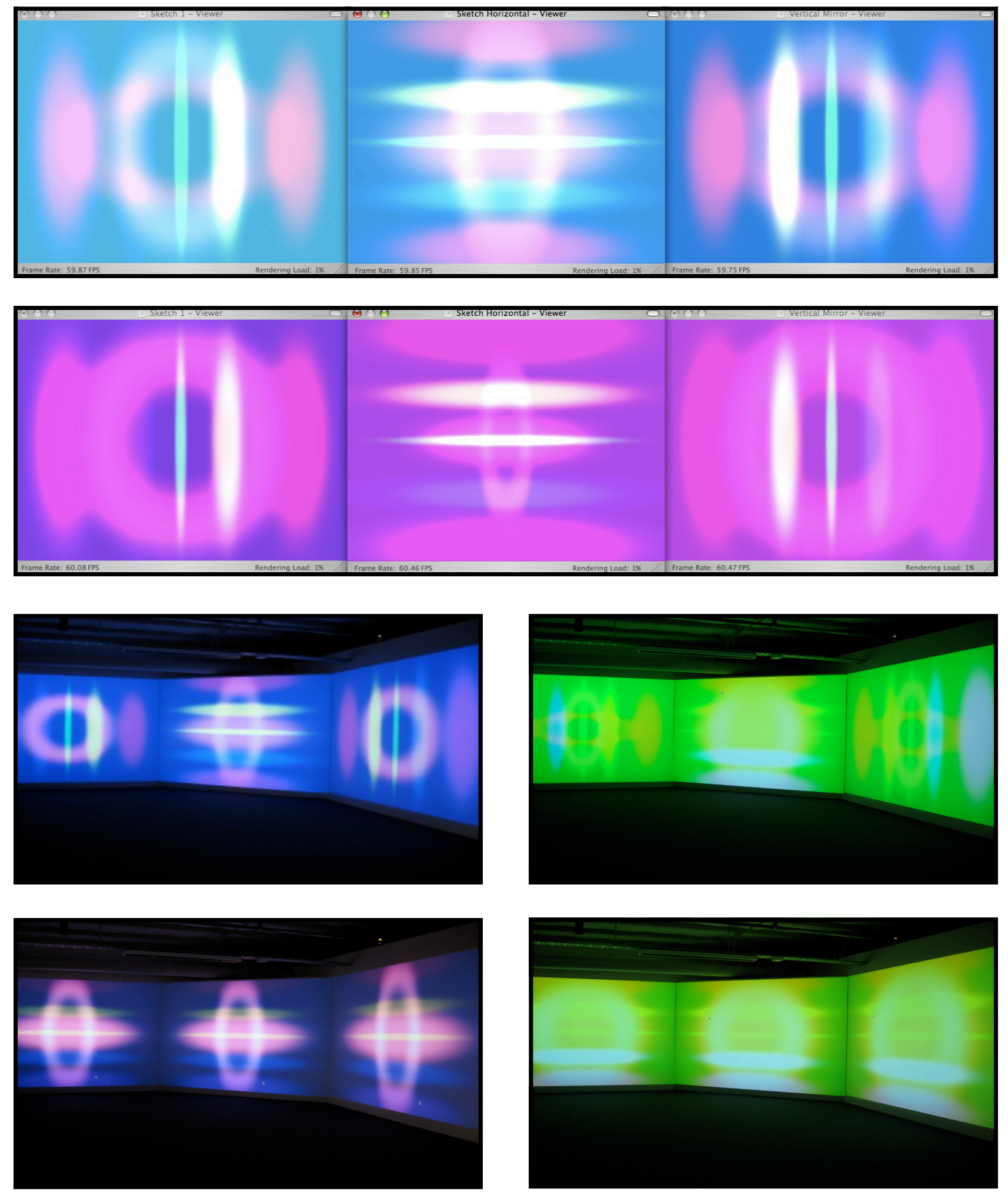
Figure $\mathbf{4 0}$

The MaxMSP/Jitter patch that interprets the data from the Theremin into the visualisations

Figure 41

The internal patch within the main MaxMSP/Jitter patch that actuates the individual layers of the themes.
The Theremin controls the visual imagery through the created MaxMSP/ Jitter patch as shown. The received data is sent to three areas of control, the 'background,' the 'focal point' and the 'theme' overlays. The background is purely a hue variation, where the focal point is the basic background shape that can be manipulated in size, shape and colour through the Theremin. From here the theme of individual strands are laid over the top, can be turned on and off, and can be varied in shape and size. Finally three layers of filters can be applied to the composition. As shown in Figure 33, these filters are activated by trigger points, when certain $x$ and $y$ values are combined.
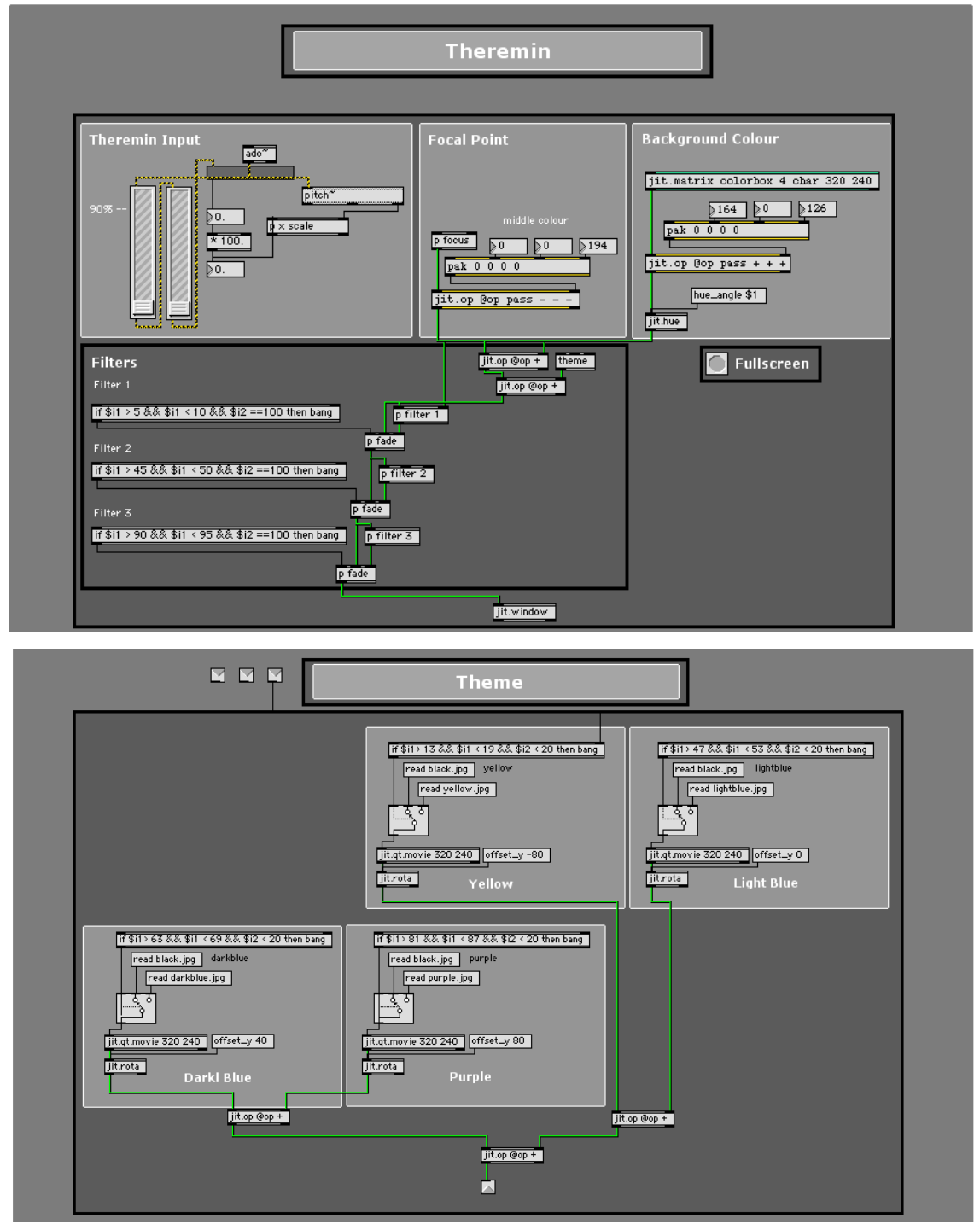


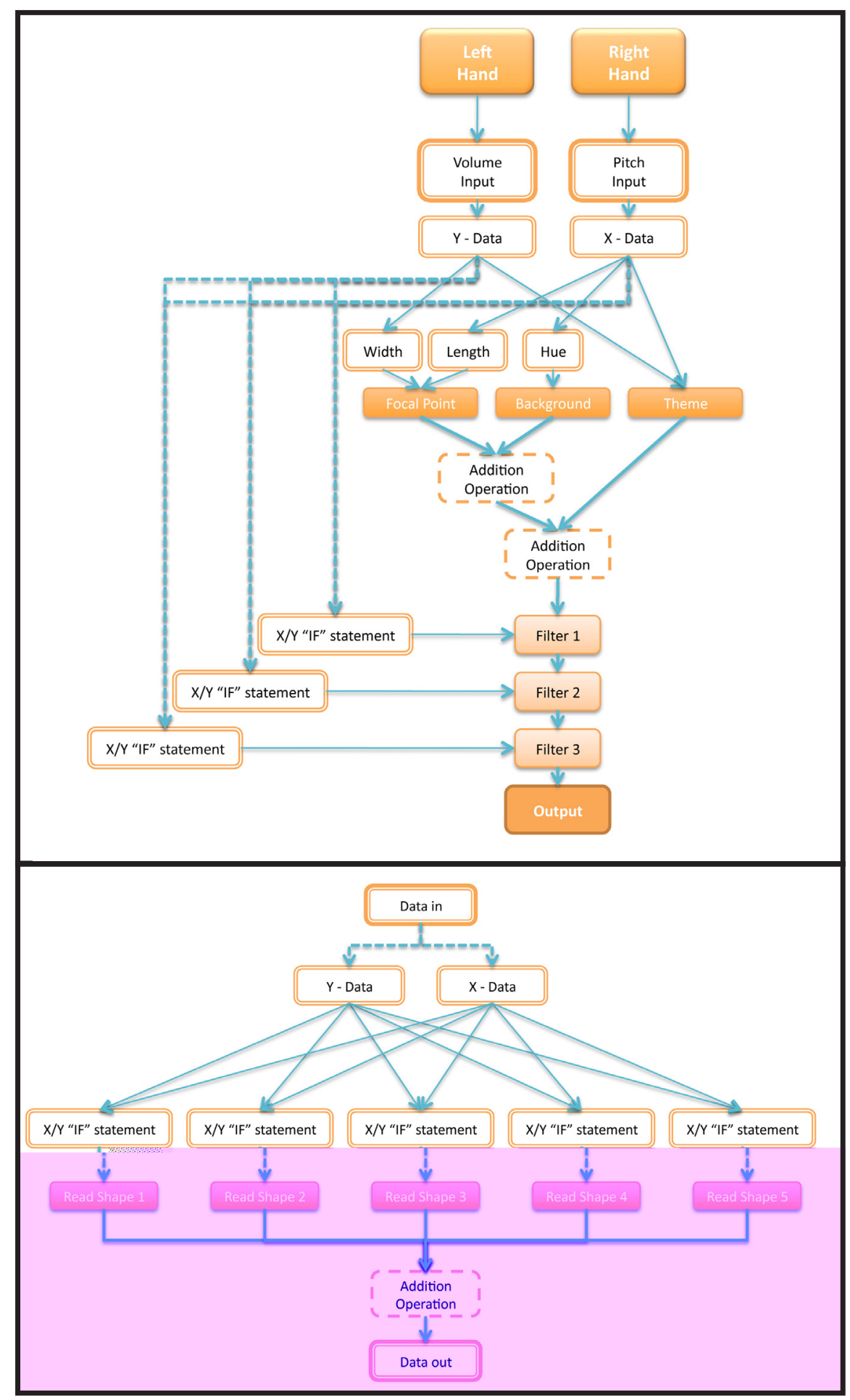

Figure 42 - 43

Diagrams outlining the construction of the MaxMSP/Jitter patches respectively
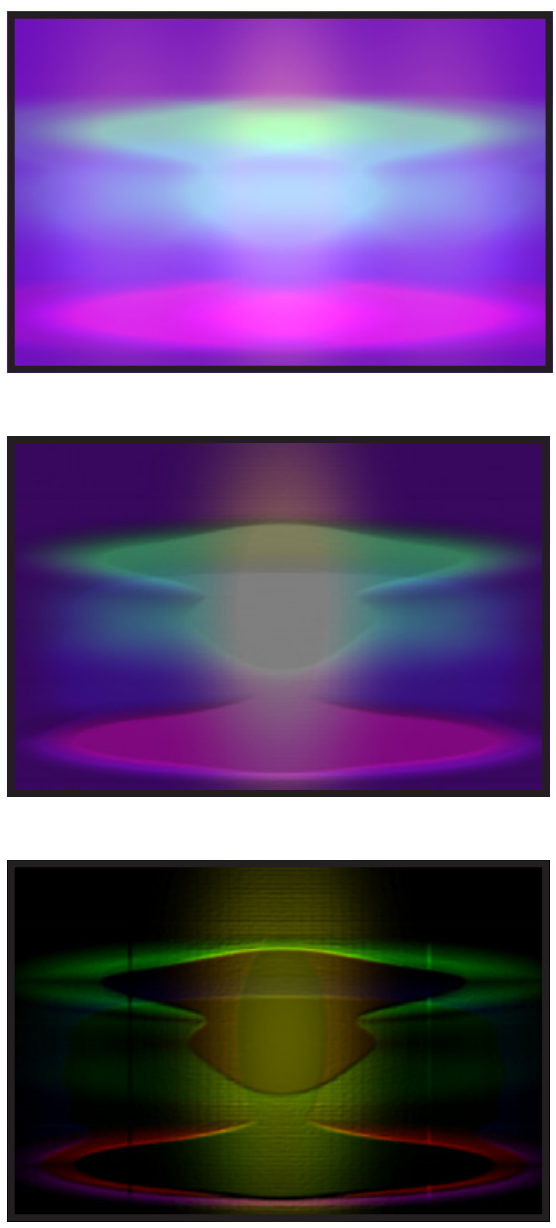

Figure 44, 45, 46

Still showing various filters being applied over the composition during the performance 
Figure 47 - 52

Stills from theremin performance
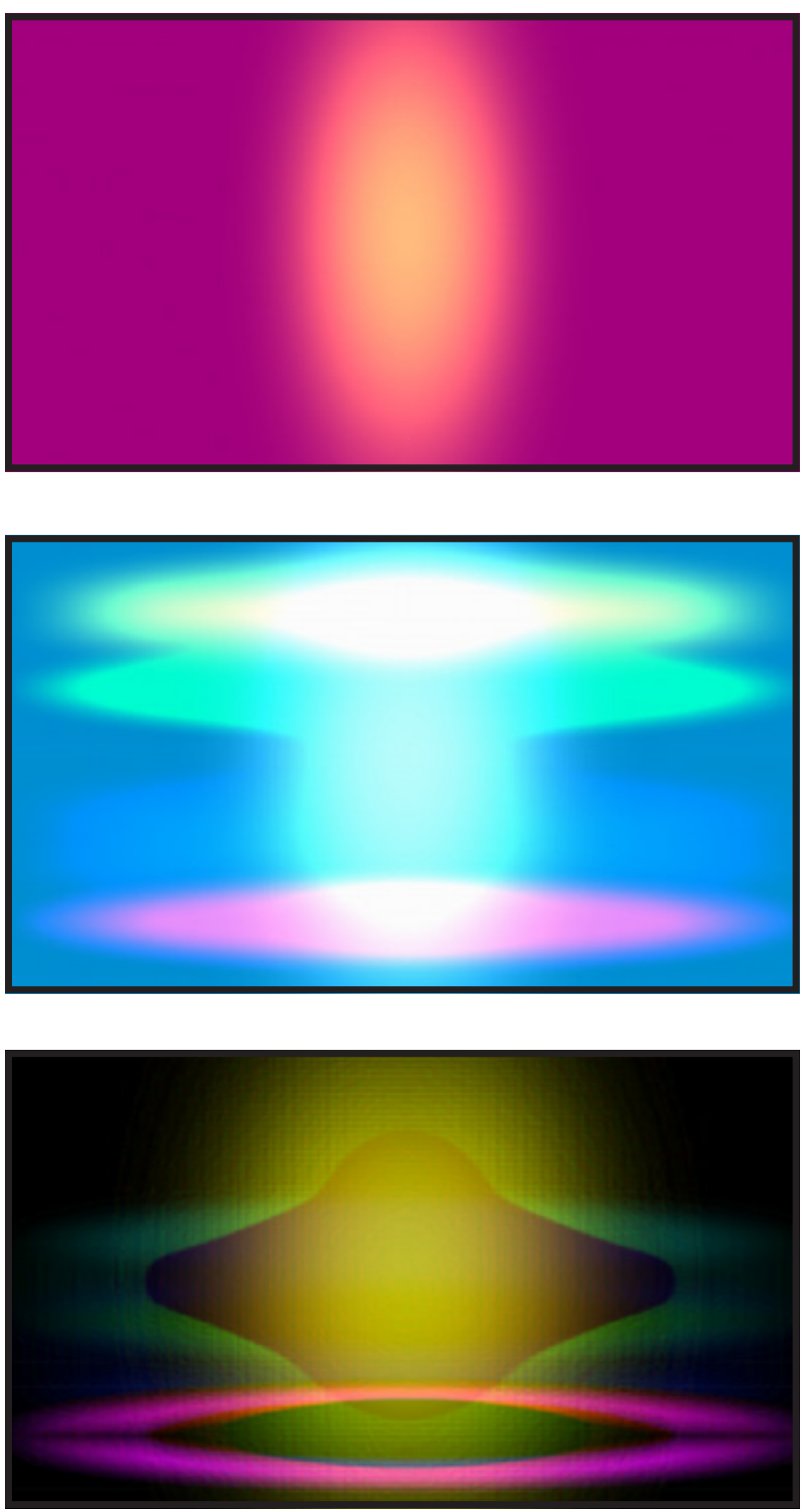

This project was completed with an experimental performance, conducted as a silent visual-music performance. The silent performance was a decision to retrace back to the original connection with synaesthesia, where the audience becomes the synaesthetic medium, translating the imagery as music on their own. This synaesthetic connection however did not prove to be as successful and does move slightly away from the intention to use this performance methodology as a response to music.
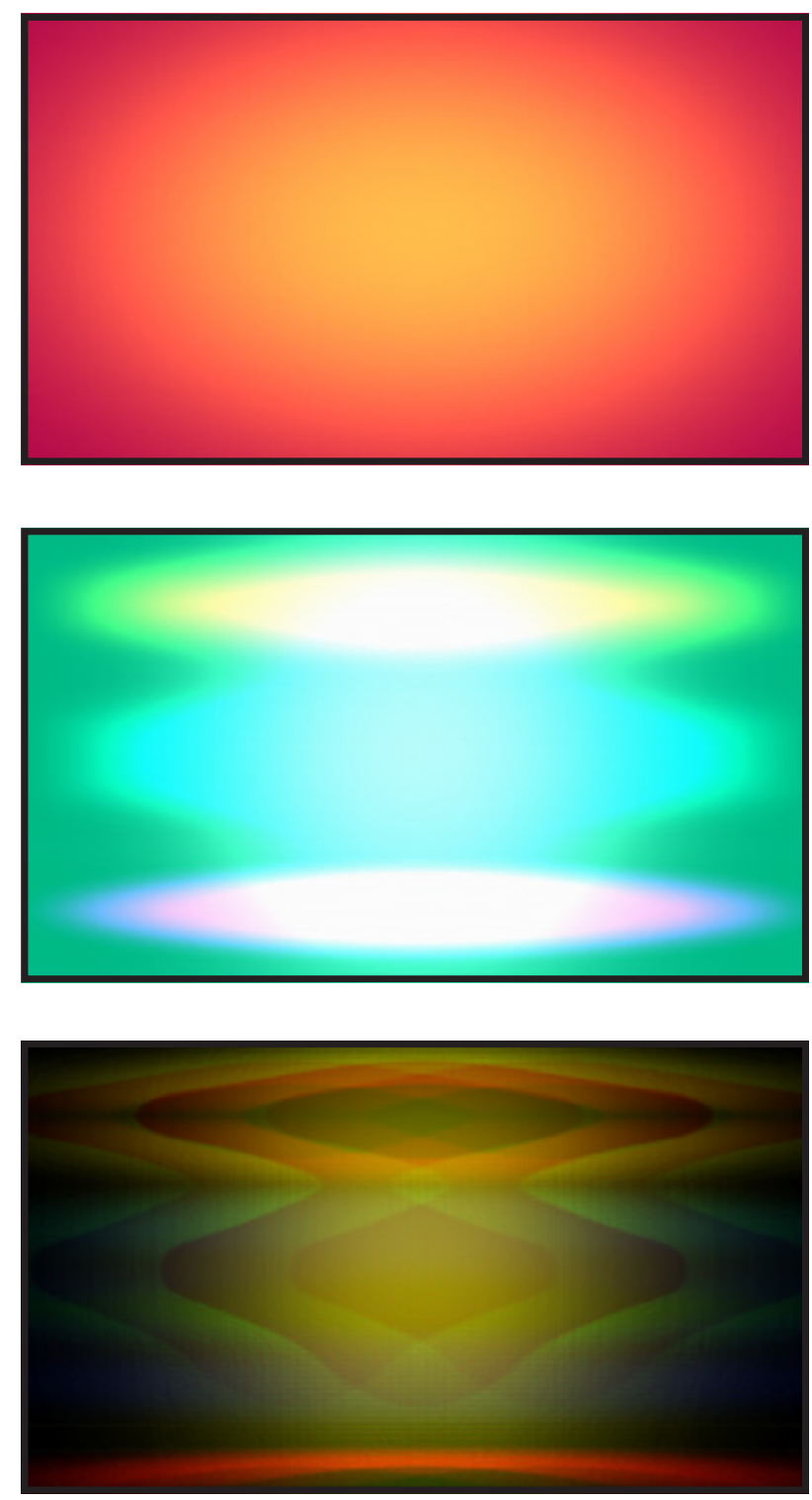

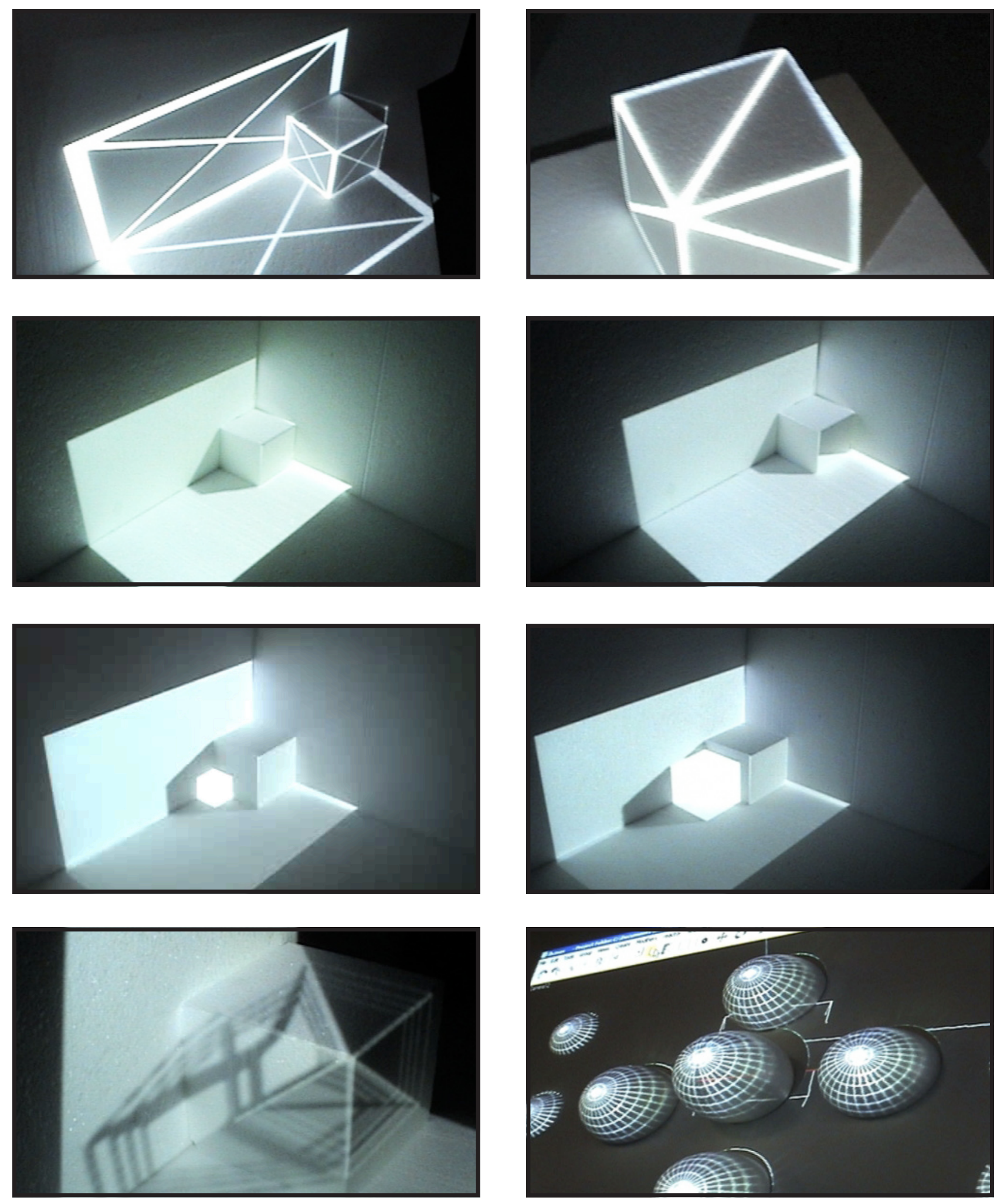


\section{Projection Mapping Experiment 1}

The intent of this experiment was to generate a Projection Mapping illusion that reveals and conceals physical space and geometry. The goal is to create a perceptive illusion purely through the use of projected light and shadow. The experiment is set out to be minimalised and geometrically uncomplicated to avoid too many external factors influencing the result.

\section{Projection Content}

The content in the first experiment is generated through the manipulation of physical space through the use of projected virtual spaces. A virtual match of the physical space is projected back onto the physical space, creating a spatial feedback loop. A virtual light source is then used to navigate the space, revealing content such as shadows and volumes that do not in fact exist in the physical space creating a perceptive illusion to the viewer. The main success in this illusion is the use of light and shadow, which tricks the viewer's eyes into believing and seeing what is not real, yet still existing in the realm of the physical space.

\section{Space}

The content created can now start to reveal and conceal space. In other words, the virtual space creates the illusion of physical space and surfaces. This is achieved through projecting fake shadows, fake cavities, solids that are purely light illusions and surfaces that seem translucent but are not. The physical space, alongside the projected content, becomes a system of illusion, where the virtual can change the perception of the physical, distorting the viewer's preconception of what is meant to occur. 

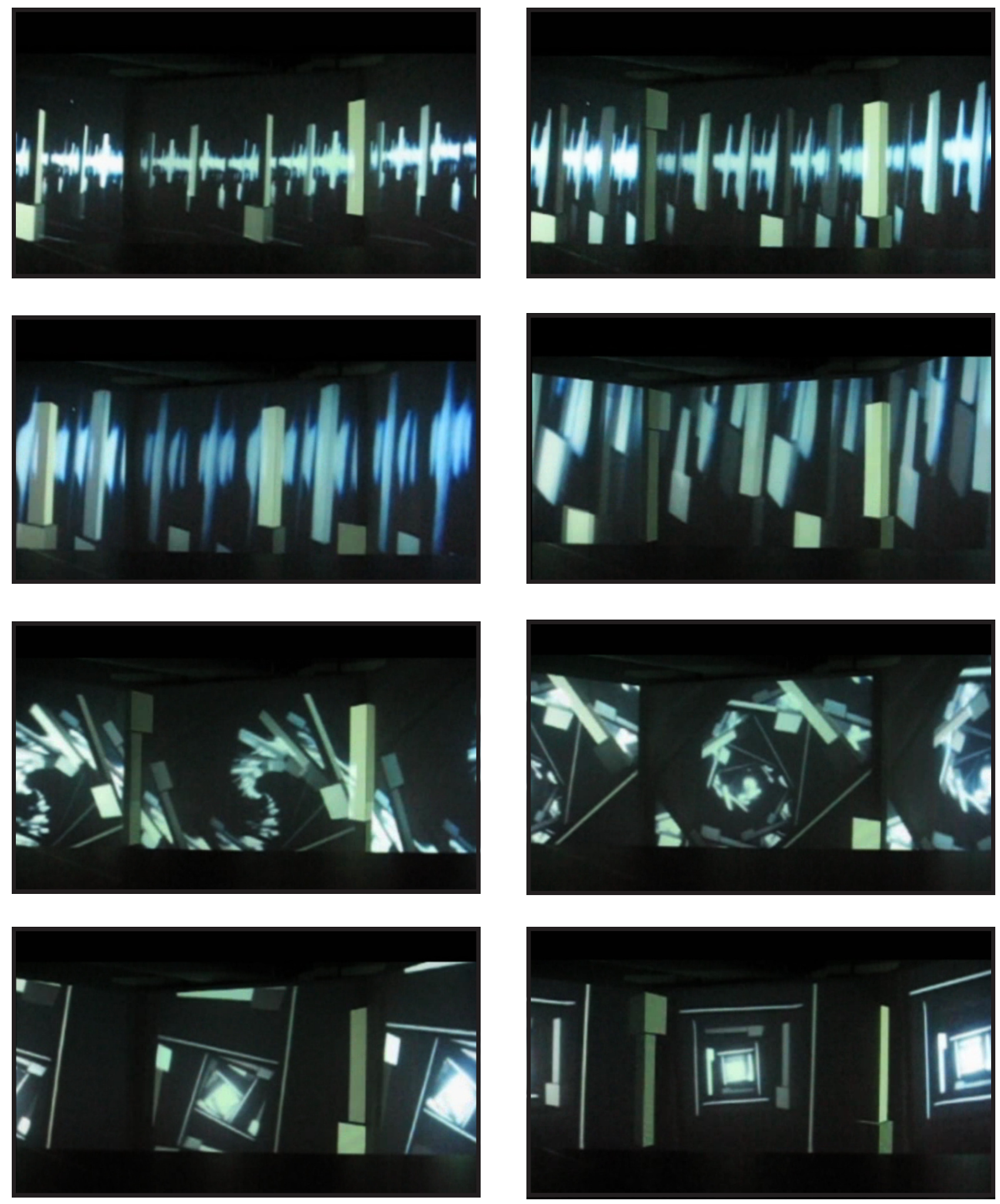


\section{Projection Mapping Experiment 2}

The intent of the second experiment takes further the idea of generating content through the use of spatial feedback. The illusion of space is created through the projection of virtual space, rather than the construction of physical geometry as in Projection Experiment 1. This is taken further to have the actual feedback loop sculpt the illusive space.

\section{Projection Content}

The scene is created from only two elements, two virtual pillars that separate the three screens. These are projected elements, created again as an illusion, but this time without the addition of any physical elements. The scene is then recorded through a camera and projected onto the actual physical planes. A camera records this projection and this recording in turn is projected again. This is very much a normal feedback loop, where content is generated from duplicating the scene over and over, rather than two similar scenes merely representing feedback as in the Experiment 1. The phenomenon differs in this case as the projections are mapped to the geometry of the space, which means the feedback loop not only incorporates the visual content but also the physical geometry. Now the space becomes part of the feedback loop, rather than purely being identified by the feedback, and therefore the spatial feedback, both virtual and physical, becomes the content generator.

\section{Interaction}

As the overall composition is created through visual feedback, the main interaction to generate movement is the camera held by the performer, which provides the means to create the feedback loop. The camera therefore offers the best tool for interaction with the loop and composition, as it can vary in pan, zoom and rotation. The camera is also a very intuitive tool that is easily understood as a process of navigation. Purely by manipulating the camera (in a sense performing with it) gives the user the ability to create a big variety of content and spatial results, through the process of 'spatial navigation.'

\section{Space}

The virtual space created from the feedback loop, moves the attention away from the physical space and creates the illusion of much larger and more complex spaces. In other words this virtual space deconstructs the physical space, generating a new sense of space, an illusionistic space. This illusionistic
Figure 61 - 68

Stills from various feedback experiments, with the focus on generating illusionistic spaces 
space, can also be elaborated as the projection mapping space or the augmented space, it is neither completely virtual nor completely physical, yet acts in unison to generate the spatial illusion.

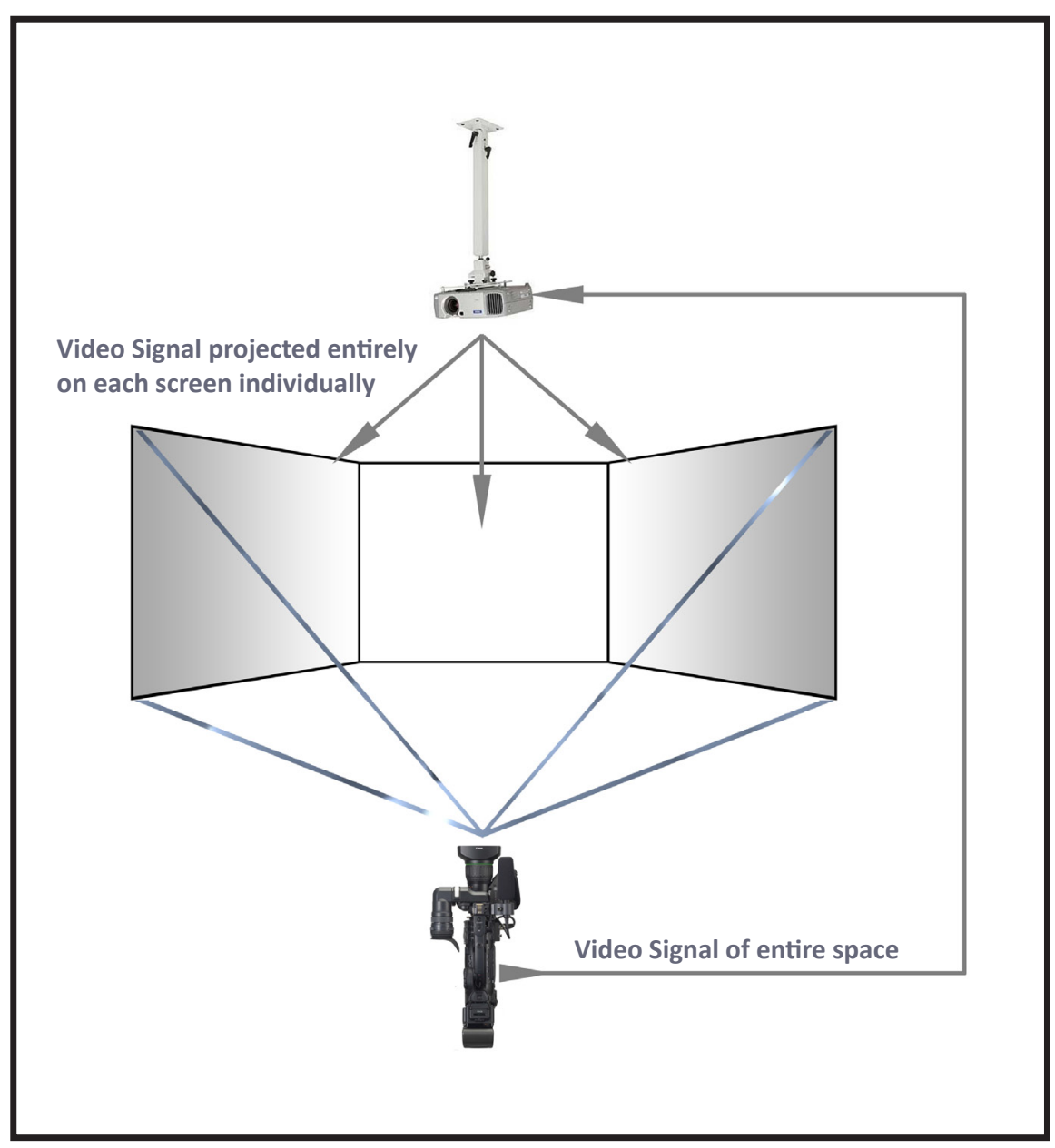

Figure 69

Diagram illustrating the use of feedback as the content creator 


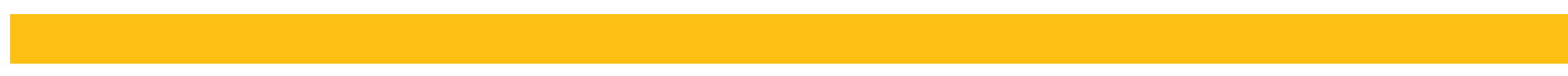



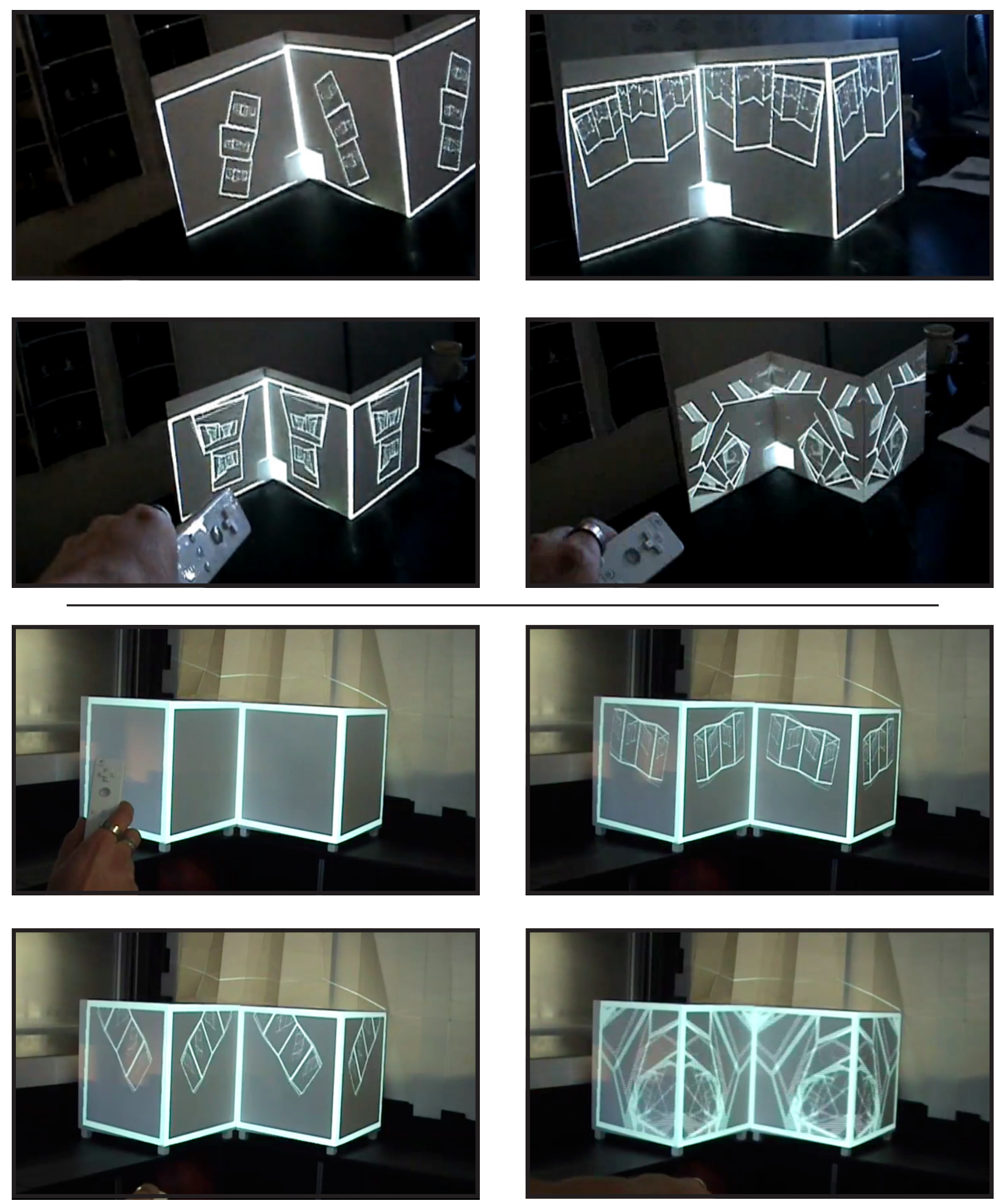


\section{Projection Mapping Experiment 3}

In this third experiment the aim was to use graphical animation to reveal the physical space rather than conceal it. A virtual scene is created identical to the physical space, and then the virtual space is projected back onto the physical space, once again creating a feedback loop of spaces. This experiment combines the use of the physical geometry of Projection Mapping Experiment 1 and the use of camera control in Projection Mapping Experiment 2 with a focus more on graphical content rather than light and shadows.

\section{Projection Content}

In this test the camera is virtual, existing in the virtual space, creating an internal loop of spaces. Geometrically identical physical and virtual scenes were created. This geometry was then revealed through a graphical component that highlights the edges of the geometry. In this experiment a virtual camera is used in the virtual 3-Dimensional environment instead of a real camera. This feed from the virtual camera is fed back into the projection onto each individual surface, creating an internal feedback loop. Fluidly generated content is created purely formed by the spaces and therefore mapped perfectly to the perspective of the physical projection space.

\section{Space}

Animations are created in these examples that simply reveal the geometry of the physical space, i.e. the edges or surfaces of the boxes. This, projected onto the physical space, creates this augmented space, which is set to be visually illusive, only revealing certain parts of the geometry. The augmented space, or projection mapping space which is the combination of virtual and physical space, and can now be considered an illusion. This opens up the opportunity to create content for a performance purely based on revealing the spaces. The animation created from the feedback, projection maps over the physical geometry, generating physical and virtual 3-dimensional content.

\section{Interaction}

By creating a virtual camera in the virtual 3-Dimensional environment, the system can offer the user a new form of interaction with the illusion. In the latter example of this experiment, two Nintendo Wii-remotes (http://www. nintendo.com/wii) are coupled to the system as an interface, one that controls the virtual camera, and one that controls the 'revealing' animation. The user
Figure 70 - 73

Stills from various interaction experiments at small scale with the use of the Nintendo Wii remote

Figure 74 - 77

Stills from various interaction experiments at large scale with the use of two Nintendo Wii remotes 
now has the ability to make the decision about what geometry is revealed with one hand, while interpreting that geometry with the controller in the other. This combination can be seen as an interaction that explores space to generate visual music content.

Figure 78 - 126

Stills from various interaction experiments
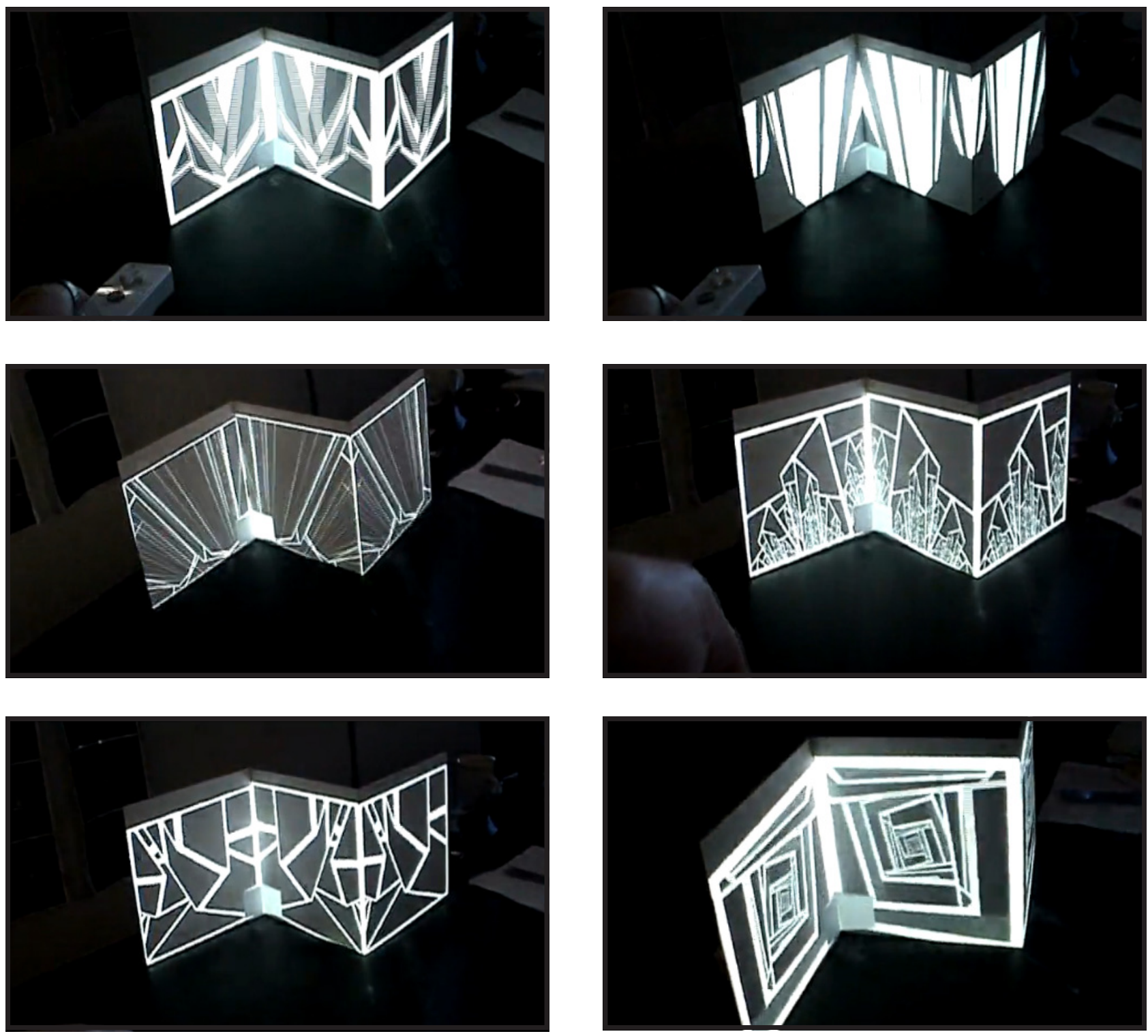

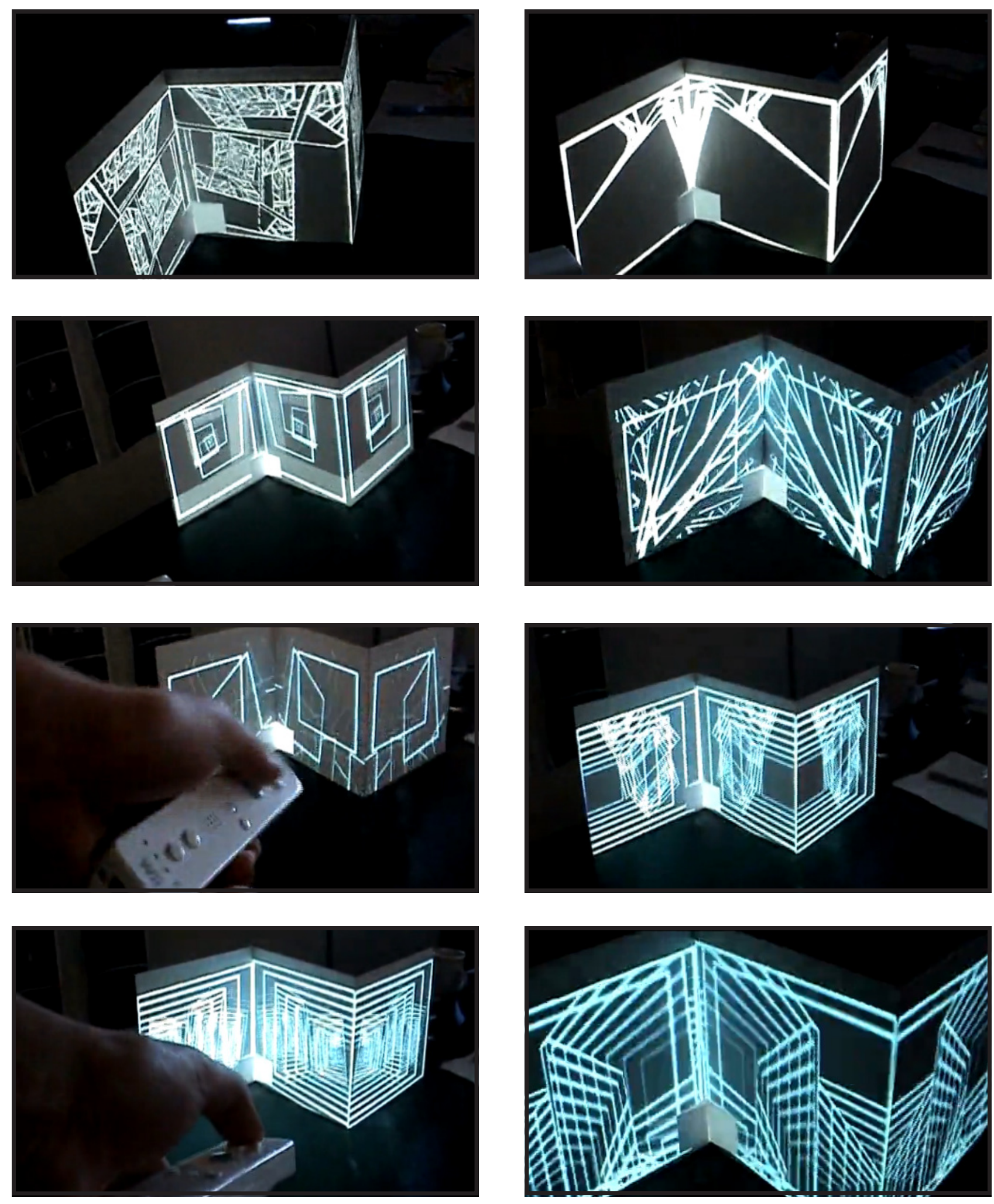

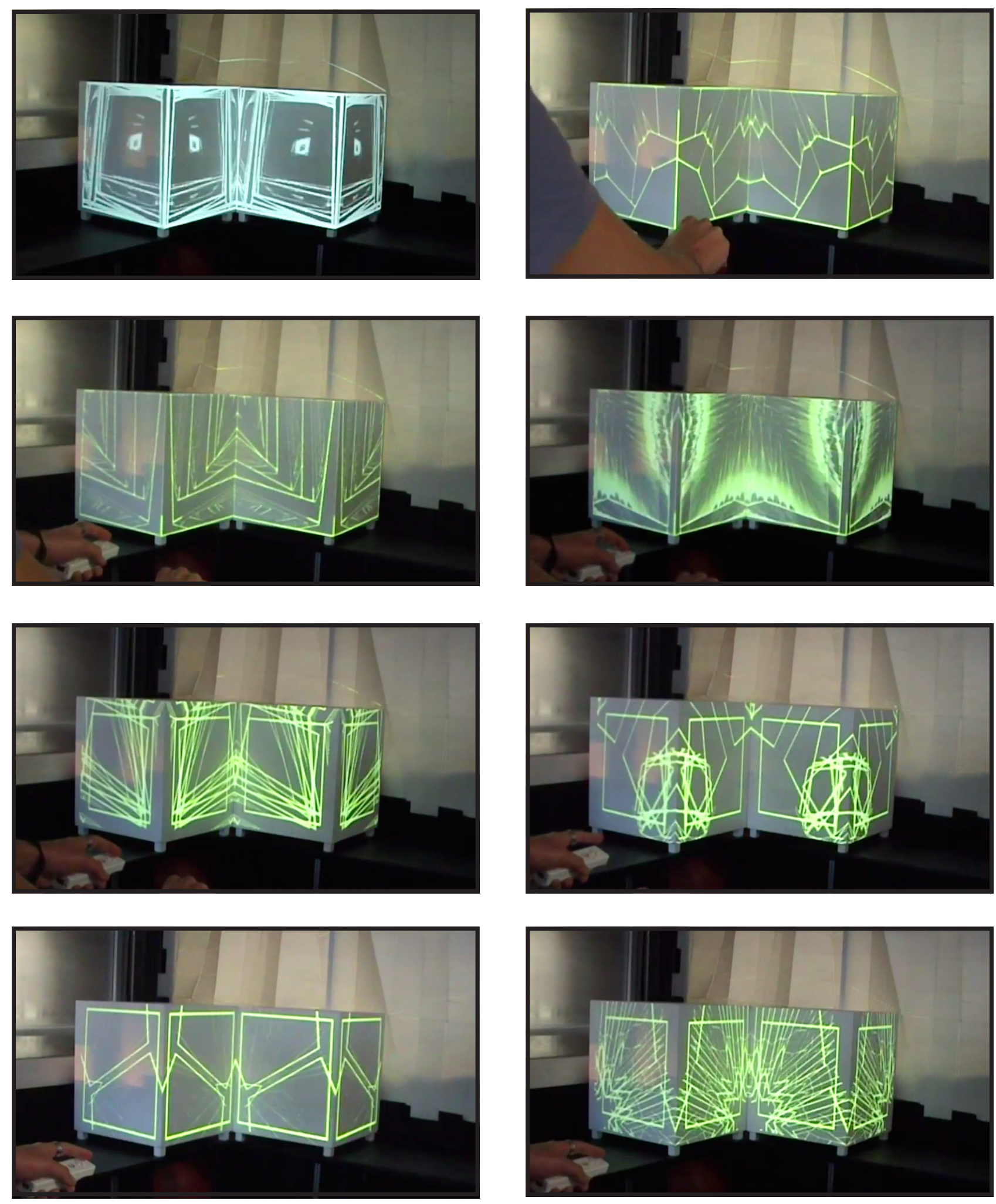

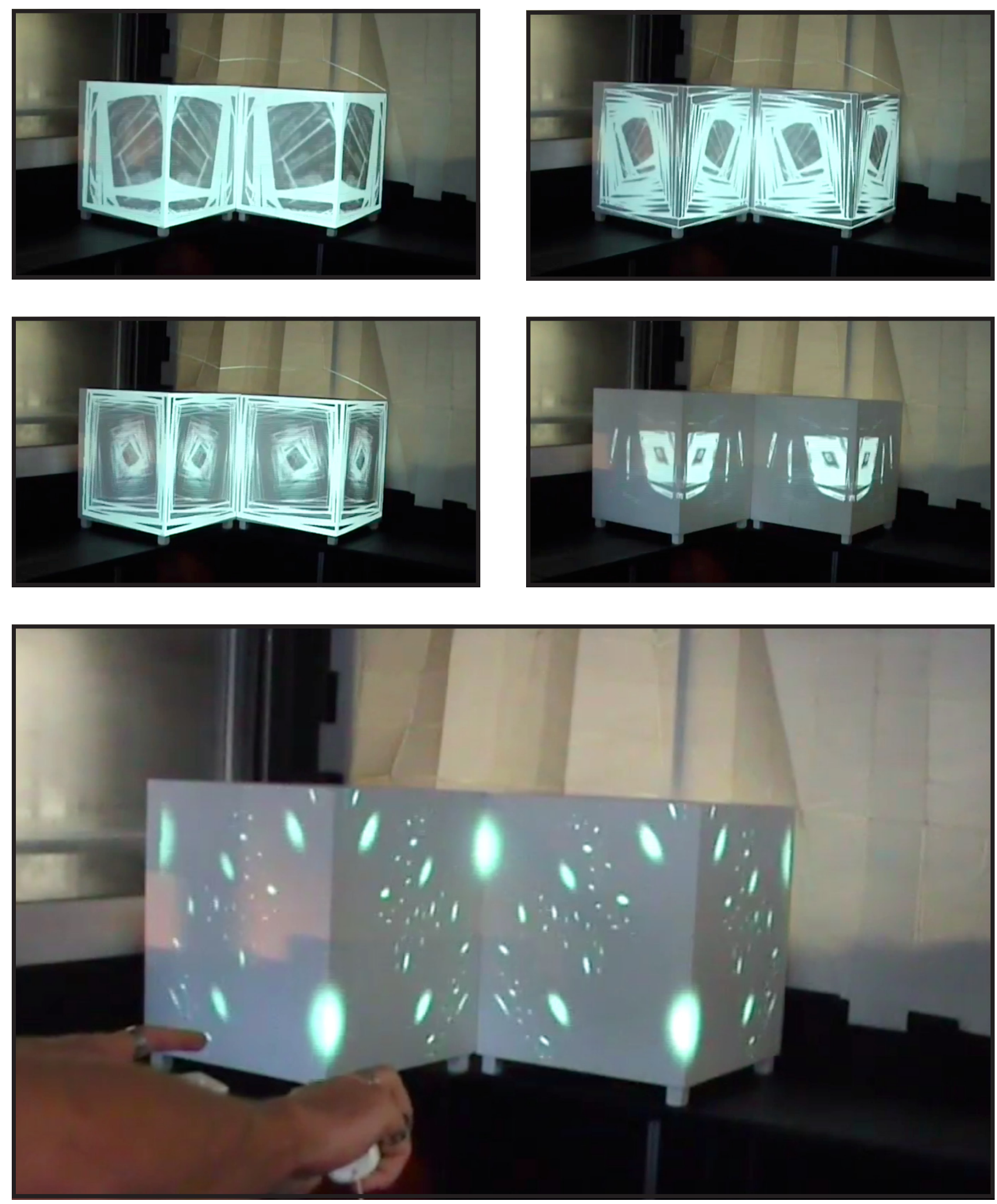

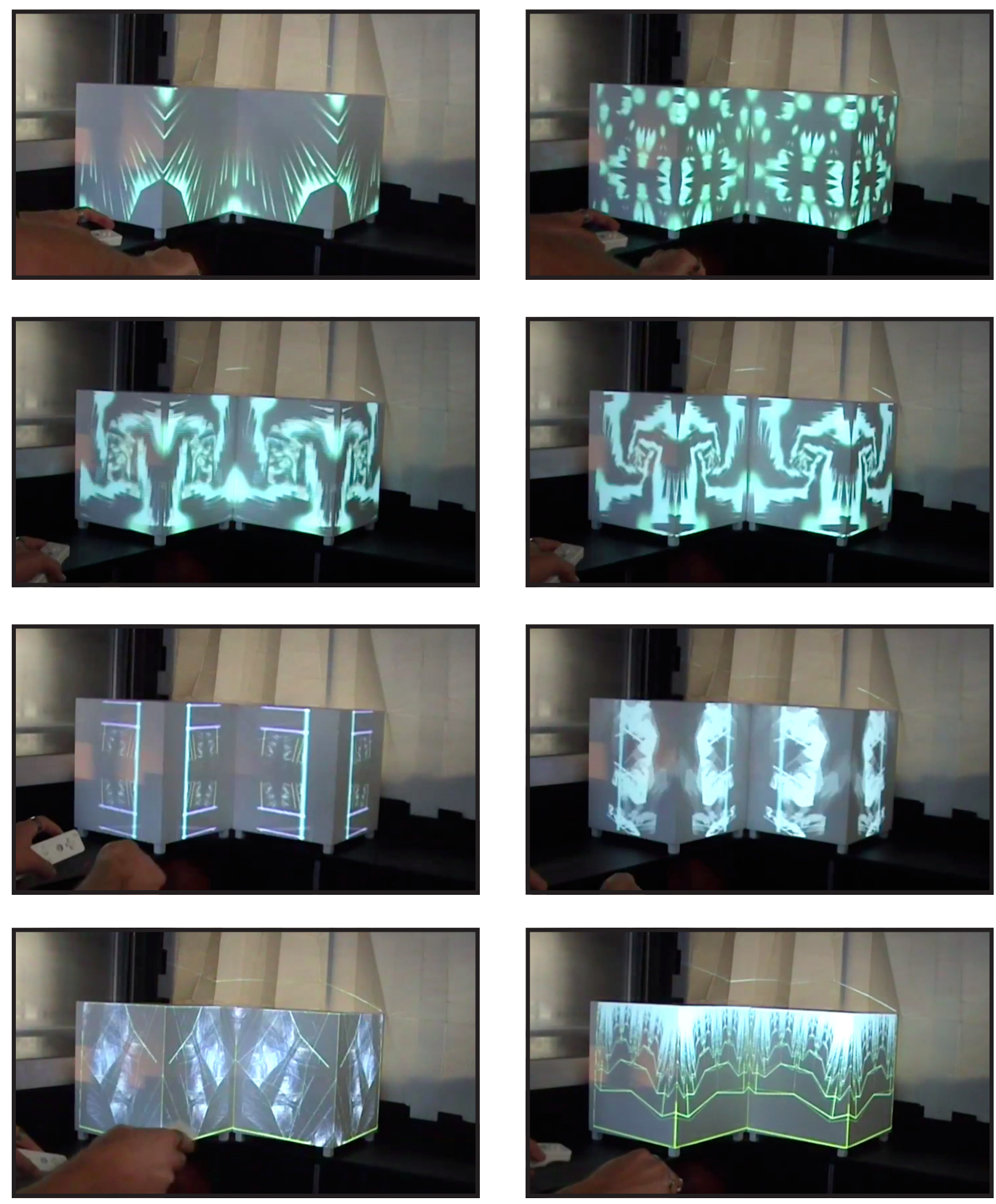

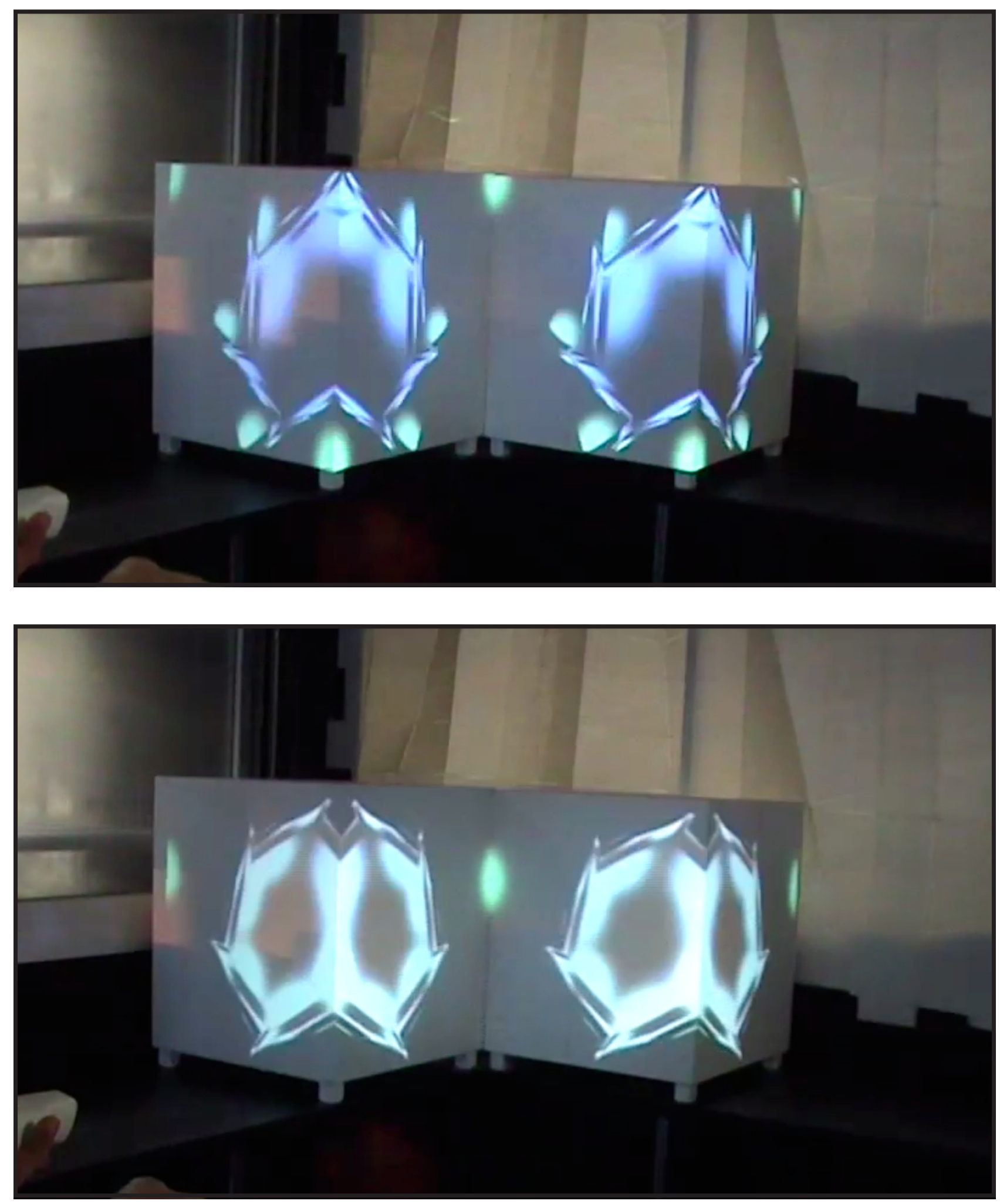

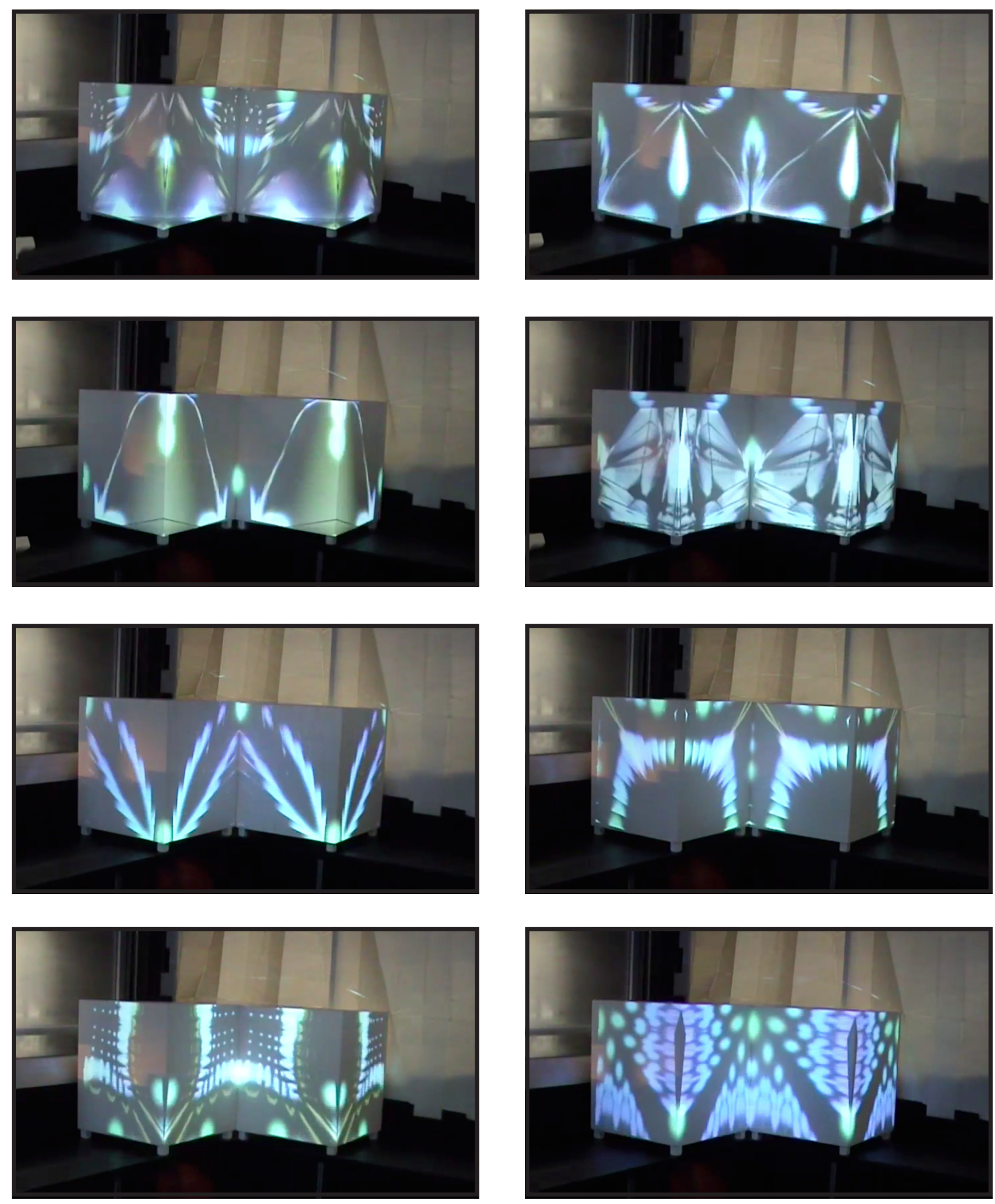


\section{Summary}

The success of the Theremin experiment lies in the understanding of gesture orientated control of visual imagery and the complex results of how the gestures work in combination. It provides the user with fluid movements and combinations, yet the user is always fully in control and aware of the movement throughout the composition. The projection mapping technique in the latter experiments has its success in the possibility of generating illusionistic situations, created by revealing and concealing certain physical geometries. Using the spatial feedback on top of this illusion not only enhances the illusion itself but also generated moving graphical content, and provided a strong secondary component that can be utilized in a performance situation. The interaction can now be seen as initiation and navigation, respectively addressing the content and the feedback illusion, where the combination of these elements create the performance.

In the next chapter this process of revealing and concealing space through feedback, and interfacing through initiation and navigation will be discussed from a theoretical point of view, to create a framework from which to design an interactive visual performance system.
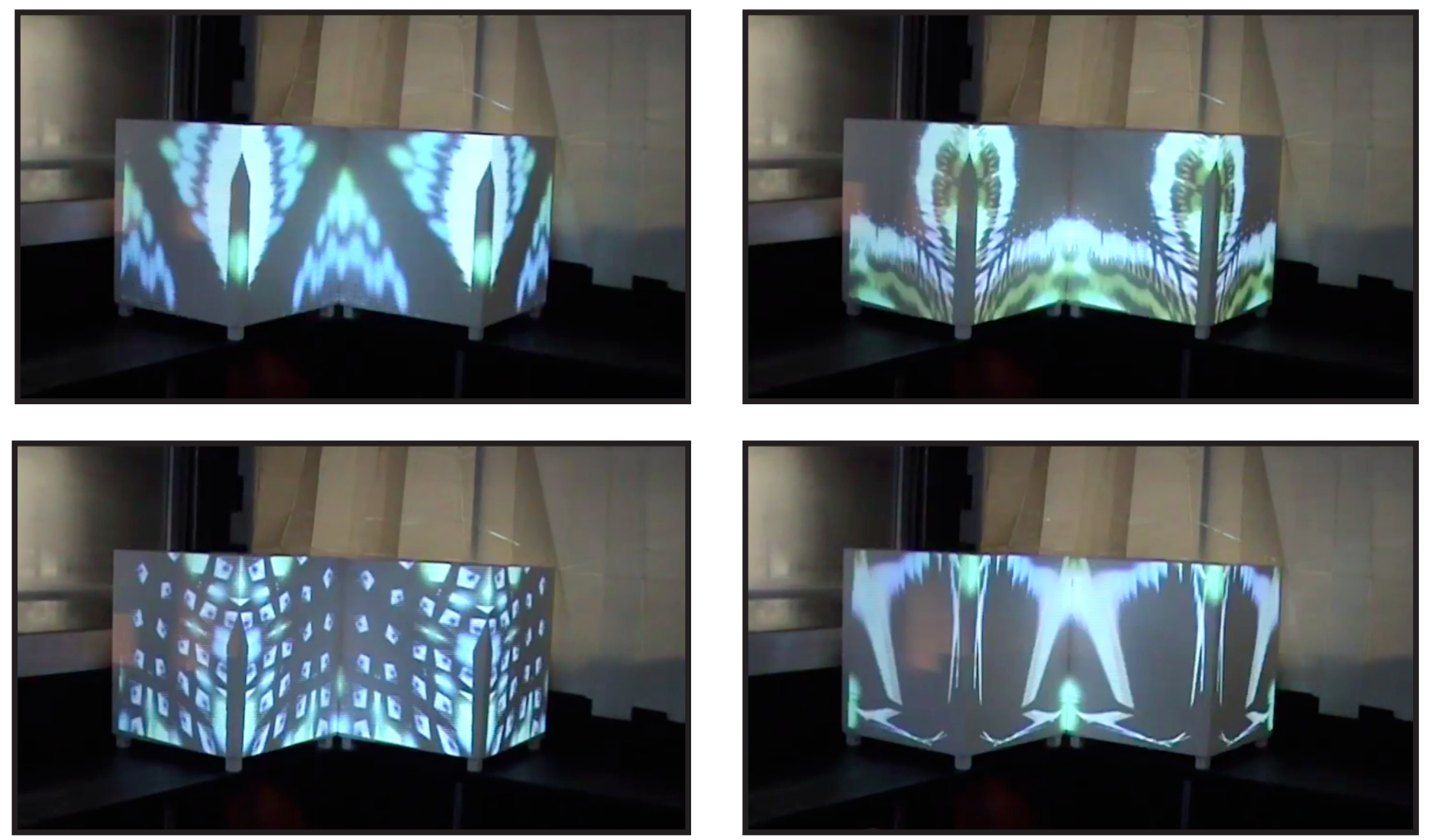
Chapter 4

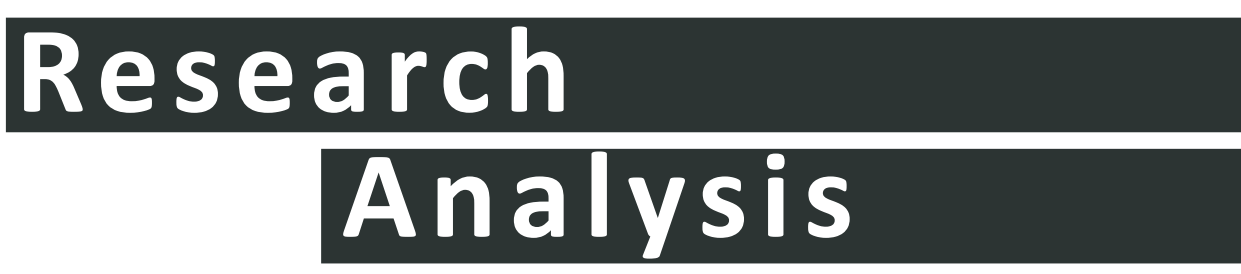




\section{Research Analysis}

The following chapter will discuss a theoretical investigation that pursues an intuitive approach to visual performances, in which the system allows the artist to respond intuitively, rather than rationally, to the performance environment. By outlining various aspects of the performance loop, a theoretical framework for evaluating visual performance systems is established, which in turn provides the grounds for creating a successfully intuitive performance instrument.

In a live set the interaction between the performer and the performance can be understood as a loop, an ongoing exchange that requires spontaneous action on behalf of the performer in response to the ever-changing environment. The investigation therefore looks at an ideal system allowing for a more intuitive approach to creating visual performances, utilizing the spatial actions and navigations of the body as the interface.

In this chapter, such a bodily response to a performance is addressed as a phenomenological interaction, as investigated by philosopher Edmund Husserl (Natanson 1973), where the spatial bodily navigation provides an intuitive interface between the performer and the performance. This spatial interaction initiates a loop: a system of continuous exchange between the actions of the performer and the visual content generated by the actions of the performer. 


\section{Towards a Synaesthetic Performance}

With technological advances in computer graphics, generative capabilities and instant access to vast media libraries, the contemporary performer has extensive possibilities of composing in real-time, making the computer an ideal tool for visual performances. Media artist Golan Levin evaluates a set of desired attributes that constitute a visual performance system; the main points being that the system as a performance instrument should have the possibility for content creation in real-time, the results should be inexhaustible and extremely variable, while the basic operations of the instrument are easy to understand, yet expressively sophisticated, and mastery seems illusive (Levin 2000). These points identify characteristics that can be applied to create a system, which can be used as an instrument for creating synaesthetic performances.

Considering that VJ performances, as part of synaesthetic performances, are defined by real-time content creation, the importance of improvisation cannot be understated. Through improvisation, much like the composition of a Jazz performance, the artist is free to create on the spot, rather than adhering to a pre-determined script. The artist is able to respond intuitively to the immediate experience, navigating through a compositional piece based on the relationship to the performance environment and feelings of the artist. The visual performance that allows for improvisation is therefore an ephemeral performance that can only be experienced at the time and place of the showing.

Site specific performances are also ephemeral performances as the performance relies on the specific characteristics of the location. Site specific performances '...aim explicitly to alter the conventional spatial practices of performance to enhance both the relationship between performers and audience and the performer's engagement with its space and site of production" ( Allain and Harvie 2006). The goal of a site specific performance is to generate an intensified relationship between the artist and the space, and the audience and the space. In order to consider, without having to prepare for, the specifics of a site, an ideal performance system would have to be flexible and adaptable. This creates a setting for an instrumental system where not only the content can be improvised but also the performance of the instrument itself. 


\section{Moving Beyond the Screen}

The computer screen can be seen as the latest development of the twodimensional canvas visual artists work on. According to new media researcher Lev Manovich, 'The visual culture of the modern period, from painting to cinema, is characterized by an intriguing phenomena - the existence of another space, another three-dimensional world enclosed by a frame and situated inside our normal space' (Manovich 2001). The computer screen is a portal to a virtual space, one that does not exist behind the screen, but rather inside the screen. The computer can render visualisations of virtual spaces that contradict the natural physical laws. A large portion of exploration in the virtual world however focuses on the imitation of the real world rather than seeking truly new dimensions (Manovich 2001). Again, parallel to the development of cinema, exploration of the abstract possibilities yields opportunity for performative intervention. The suggestion is to approach the visuals spatially, as we would interpret sound. Surely the performance of a musical piece exists spatially rather than being confined to the loudspeaker, the means through which the sound is produced. The visual image therefore, can not be confined purely to the screen or even projector, the means through which the image is delivered, but more the spatial experience and opportunity it offers for visual immersion.

A spatial visual experience of immersion can occur once the virtual dimension and physical human space reaches the same size. Up until recently this has been demonstrated in Virtual Reality (VR) by breaking away from the screenbased connection and immersing the viewer completely in the virtual through head-mounted displays. Manovich (2001) establishes this as a '...radically new relationship between the body of the viewer and the image,' where the viewer is now immersed in a virtual world at the same scale to the real world. The similar scale at which the two worlds are operating creates a potential to blend the two worlds. In a direct simulation of the physical world the user is able to react to the virtual environment, in a spatial fashion similar to that of the physical world. The user is free to move within physical space, which translates, into the virtual space. VR technology is still very restrictive as although it has matched the spatiality of the human world it outright disregards the physicality of the human world. VR restricts the physical exploration by completely absorbing the user in the virtual world through the visual but not through physical tangibility. A variant technology that addresses this virtual/physical relationship is Augmented Reality 


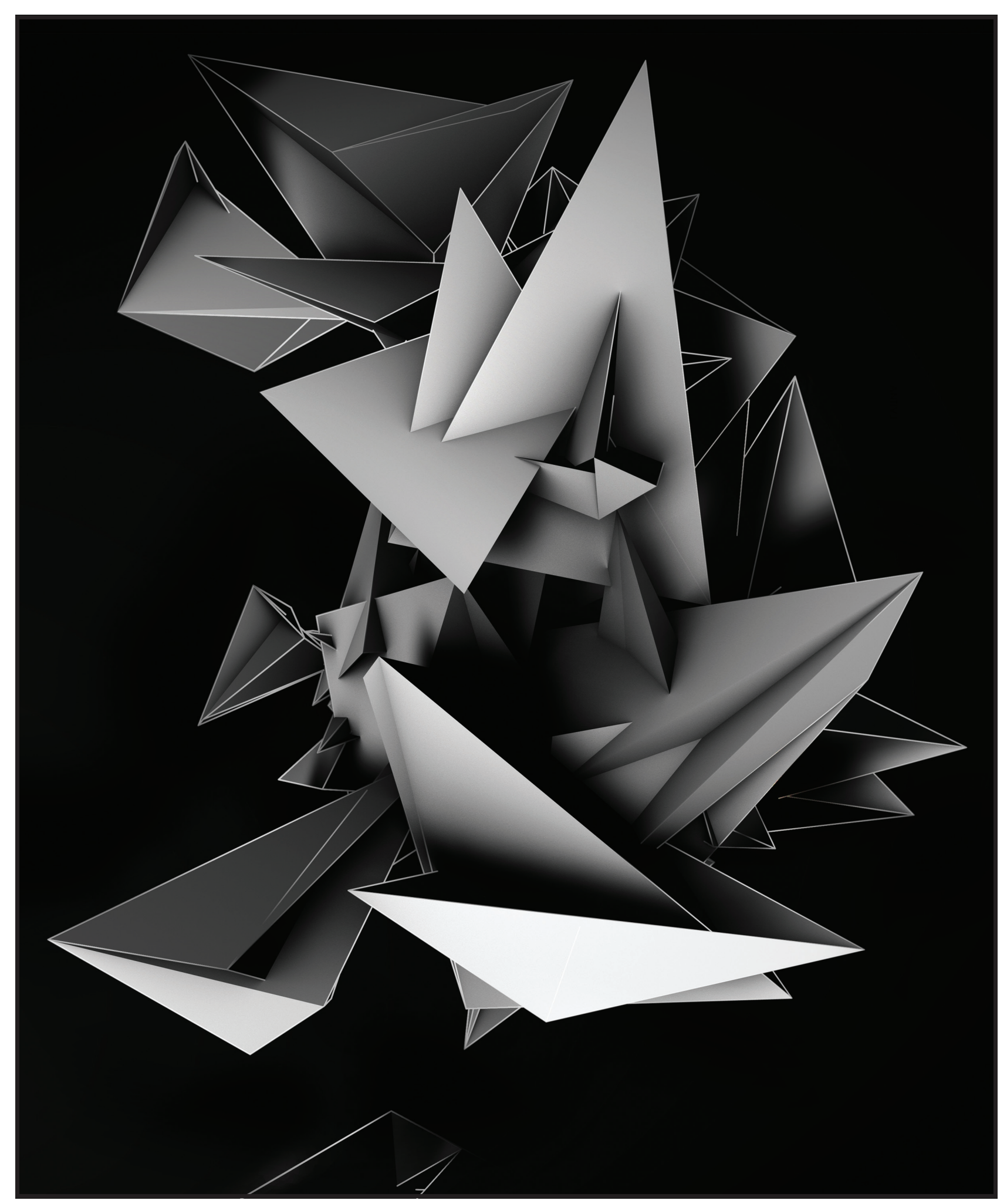

Figure 127

AntiVJ - http://blog.antivj.com/ 
$(A R)$, or what is often referred to as mixed reality, where the virtual is instead overlaid onto the physical at the same scale, an experience that can be shared amongst multiple viewers.

AR takes many formats, in technology as barcode detection and in artistic practice as projection mapping. Manovich (2001) describes Augmented Reality as the 'overlaying of dynamic context-specific information over the visual field of the user.' Now the simulation, as Manovich describes, merges the virtual and physical space, where the user can actively move around the physical space, while interacting with the virtual (Manovich 2001). Specifically in Projection Mapping, the unification offers the artist an opportunity to generate a spatial visual experience, operating both in the physical and virtual environments. This blending however does not just affect spaces but also the actions that take place in the spaces. Actions within the virtual space, that are not possible in the physical dimension, are now portrayed back on the physical medium. This opens the possibility to perform illusionistic tasks that are based on deconstructing the physical space through virtual actions. The frame analogy of the window into the virtual world is no longer sufficient, as both the virtual and physical can now exist in parallel perception. Through Projection Mapping, what was inside the screen replacing reality now exists in the physical as a supplement to reality.
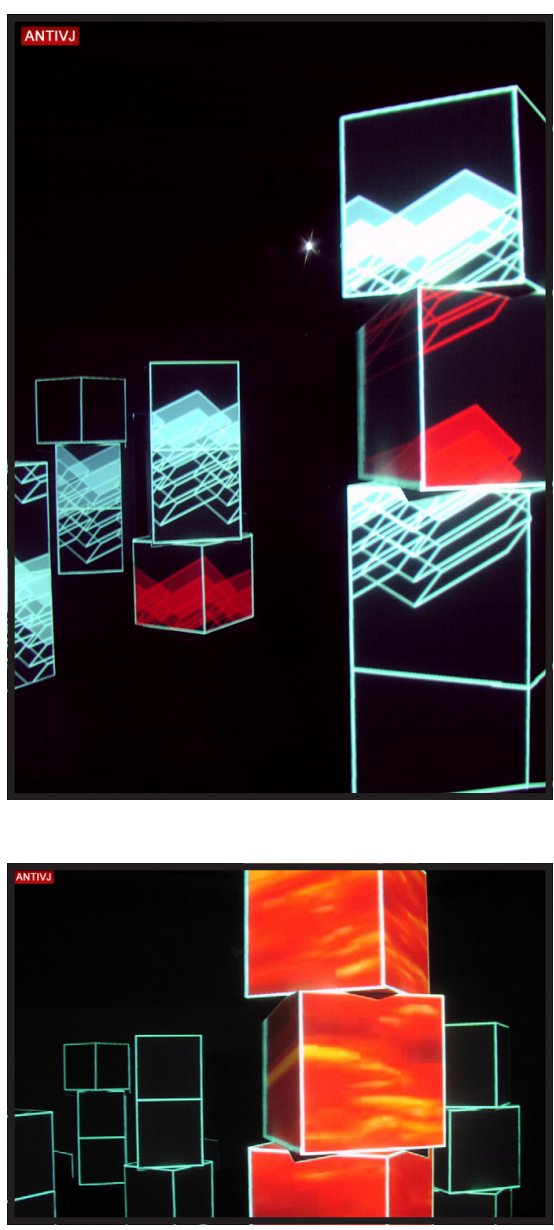

Figure 128, 129

AntiVJ 2007 Mapping Festival Cube Mapping Installation 


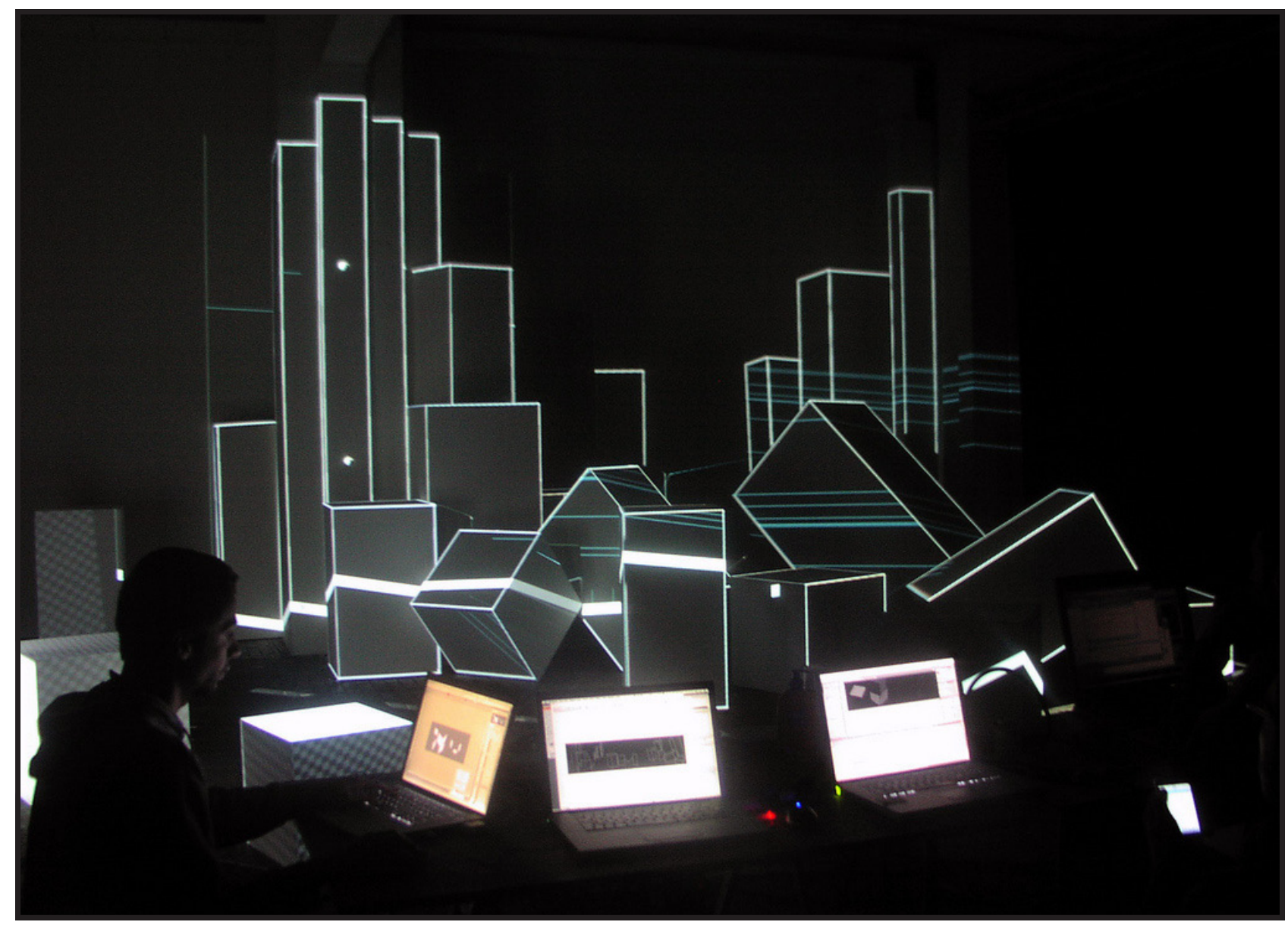

Figure 130

AntiVJ 2009 - Installation

Watershed Showcase, Bristol 


\section{Space Medium}

Augmented reality, in particular projection mapping, bridges the gap between the physical and virtual space, making the virtual physical and vice versa. The perception of this spatial continuum (Manovich 2001) lets the viewer experience two worlds occurring at once in the same place and time, but with varying physics. The image that is being displayed is still only one of illusion where, as Manovich (2001) describes 'The ideology does not demand that the subject blindly believe it, ... rather it puts the subject in the master position of someone who knows very well that she is being fooled, and generously lets herself be fooled.' The illusion cannot be interpreted as reality but rather a way to experience reality. An experience based around the compositional relationship between the spaces.

The relationship between matter (objects) and space is such that one cannot exist without the other. Pavel Florensky (quoted in Manovich 2001) explains that ' $(t)$ he space-medium is objects mapped onto space... We have seen the inseparability of things and space, and the impossibility of representing things and space by themselves.' Taking Florensky's analogy and comparing it with AR where the virtual space is mapped onto the physical space, it can be said that the virtual space becomes the 'matter,' and the composition of spaces becomes space-medium. The composition is then the same space existing in two formats overlaid on itself, hence the composition, as the medium is a self-informing, self-generating medium. The Projection Mapping composition now exists as a medium in itself, generating its own laws and rules of behaviour, which in turn only apply to itself. This form of spatial feedback creates an illusionistic mirror that is generated by reflecting a world back on itself.

A parallel to this concept of spatial feedback can be seen in the techniques of two-dimensional video feedback. Video feedback is a digital image displayed on a screen, filmed externally and fed back into the original image as a composition. As the process is ongoing, the composition becomes an organically multiplying image. Gene Youngblood describes the effect of the feedback techniques used by Robert Zagone in Videospace as follows;

The result is an almost visceral, physical quality to the image as endless waves of flaking matter peel away from the silhouette, 
slowly at first, then faster and more chaotically, with ever-increasing convolutions of geometrical patterns. A kind of serial nightmare, like a magnetic field suddenly rendered visible, the reverberations of chiaroscuro flip hectically in giant sweeping flak bursts of light until shimmering white glow is all that remains of the image of a man (Youngblood 1970).

Although this feedback discussed concerns two-dimensional video feedback the analogy remains the same, where the actual image is produced purely from the existence of the composition in the first place. The medium is the medium, a composition that informs and creates its own ephemeral existence through feedback. This feedback discussion can now be applied to the composition of the virtual and physical spaces, where the dialogue between the spaces becomes the content generator. This new dialogue between the virtual and physical exists because as Manovich paraphrases Jeffrey Shaw '... the virtual can at least preserve the memory of the real it replaces, encoding it's structure, if not it's aura, in a new form' (Manovich 2001). This new form of feedback between the spaces creates an apparent loop, which opens up an access point for the artist to manipulate the content. 


\section{The Loop}

Pre-cinema instrumentation in the nineteenth century, from the Thaumatrope spinning disk through to the Kinetoscope as the precursor to cinema, were all based on a loop. As the instruments were mostly cranked by hand, they had a very definite object/viewer relationship. Each looped scene depended on the actual physical interaction of the user that could vary speed, and fluctuations in speed, placing these instruments in the realm of performance. As the cinematic era started all these looped visual performances lost the physicality in interaction in order to avoid what was seen as limitations. The 'Live Cinema' movement has re-invented this role of interaction in contemporary cinematic practice, where digital video loops are controlled in speed and placement upon the screen. This looped concept, which has its roots in pre-cinema, establishes itself not only as a graphical content generator but also as an interaction between the user and the instrument.

Figure 131

Diagram outlining the various loops within the system and how they are intergrated

\section{VISUAL PERFORMANCE SYSTEM}

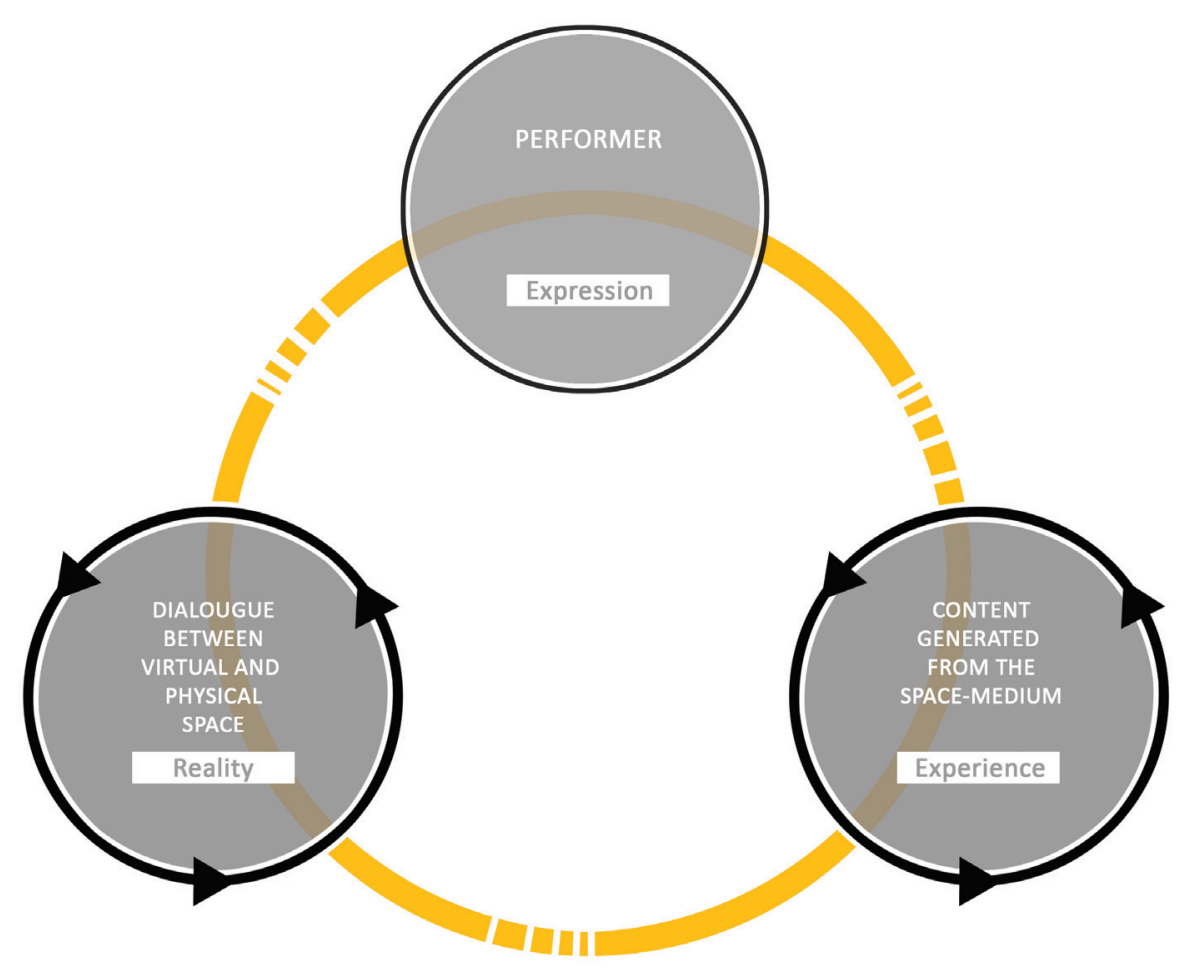


This loop can now be discussed as a concept for interactivity; interactivity between the performer and the content but also between the performer and the space of the performance. Media artist David Rokeby analyses interactivity as a loop in which the actions of the performer are not only reflected by the system but also refracted, providing a mirror that establishes a relationship between the self and the experienced world (Rokeby 1995). Creating this 'loop' between the performer and the space is the main elaboration of the visual performance system, where deconstructing or refracting this loop, varying the conditions under which the loop exists, then generates the performance.

The loop can be found in visual performances on three levels; the loop as the dialogue between the virtual and physical space, the loop as the 'spacemedium' or content generator and finally the loop as the interaction with the performance. As this interaction loop exists within a spatial context, the structure of navigation, as all encompassing rather than linear, has to be considered as the conceptual paradigm (Manovich 2001). Rokeby suggests that '...(t)he sequence in which the spectator (or performer) experiences these vistas forms a unique reading of that space' (Rokeby 1995). If the spaces are navigated in a linear fashion it will be experienced in a linear fashion. However, if the spaces are presented through an all encompassing navigation it will be experienced as such. To visualize this navigation, the virtual camera, as the means through which the digital image is displayed, becomes the intermediary between the virtual and physical space (Manovich 2001). This virtual camera offers a tool for the performer to receive visual feedback on the actions enacted in the performance. By controlling the virtual camera the performer's gestures are directly reflected back to him. Levin adds that these gesture capturing techniques in which the performer's actions and spatial properties are played back, open up '...tremendous capacity to produce lively, organically-animated results...' and places value on the '... exceptionally tight relationship it establishes between the user's input and the system's output' (Levin 2000). The part of the interface that controls this function therefore needs the ability to be used in the same manner as to which it would respond in the virtual (Krueger 1991). Levin goes on to suggest that coupling the user to the environment through such direct control, offers the ability to manipulate movements quickly in response to the physical signals, '...forming a tightly closed control loop' (Levin 2000). 


\section{Instrument as Metaphor}

This system identified by 'the loop' needs to be discussed on its own merits as a visual performance instrument. The interface to the system should respond intuitively to the actions of the performer, which will in turn allow the performer to respond to the system intuitively with his body rather than through an analytical process. Edmund Husserl investigated phenomenology as a perception on the world that responds to the body rather than the rational mind (Natanson 1973). The identified visual performance methodology so far generates visual content in a spatial immersion, where the artist responds to the entire environment through spatial bodily navigation. In such a phenomenological situation, the artist is able to make an intuitive response to his environment and his own performance, creating a fluidly organic loop where the output is discovered rather than predicted. In Artificial Reality II Myron Krueger lays out a prime motivation for creating such generative interactive systems: 'Indeed, one of the strong motivations guiding this work is the desire to compose works that surprise (even) their creator' (Krueger 1991).

In Levin's words, the feature of a successful intuitive instrument is '... that its results are inexhaustible and extremely variable, insofar as it can afford many different kind of compositions, and is sensitive to subtle features of a user's performance' (Levin 2000). The term instrument can be used very generally here, applying for example to a simple interface such as an ordinary pencil, as well as to a musical instrument such as a classical violin. An effective instrument explores simplicity in use and complexity in possibility. There is not a direct need for the artist to understand the specific mechanics of the instrument but more the performance possibilities the instrument provides. The instrument, as a symbol for interactive work, therefore has to include some form of learning and experience mechanism (Rokeby 1995). In order to perform any instrument with skill, practice is required where the user is able to explore the dynamic opportunities as well as the limitations. Levin suggests that in an effective system '... the experience of accustomizing oneself to these rules, if these rules are regular enough, can be quite enjoyable (Levin 2000). 


\section{Summary}

\section{Conclusion}

A well designed visual performance system needs to be one that addresses simplicity in use yet is complex in output, providing the artist with an opportunity to use the system as an intuitive instrument. In contrast to the approach provided by navigating standard software interfaces that are not always easy to use, the intuitive nature of this proposed interface allows the artist to respond fluidly to the environment, to his own actions and to the experience itself. This proposed environmental response negates the need to navigate software interfaces, rather allowing the artist the ability to explore the creative possibility of the instrument.

The concept of the 'loop' as the principle interactive structure for the instrument forms the framework for this visual performances system. It is analysed on three levels, as a dialogue between virtual and physical spaces breaking away from a screen based analogy, where the integration of the spaces offer opportunity as the source of the content, and as an intuitive interaction through visual feedback between the performer and the intended performance. The instrument uses a phenomenological approach to interfacing where a bodily responsive interaction exists between the system and the performer. This approach therefore focuses very much on the spatial notion of the visual performance allowing the artist not only to respond to the environment itself, both physical and virtual, but in turn to his own actions within the environment. Focussing the design of a visual performance system on the principle of interaction, rather than the manipulation of complex software will provide the artist with a much more intuitive organic visual instrument.

In the following chapter, I will discuss the design criteria that will define the framework for the design of an intuitive visual performance instrument, generated through the use of spatial illusion. Each of the principles identified within the research to this point is evaluated in a set of criteria, vital to the successful design of such an instrument. 
Chapter 5

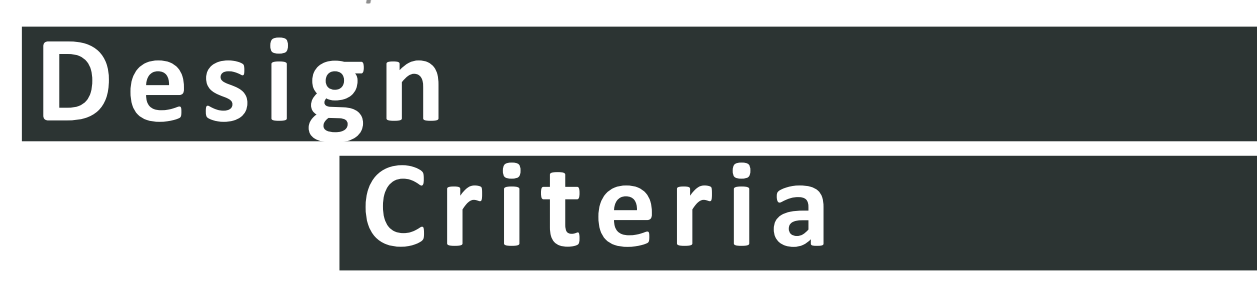




\section{Design Criteria}

From the framework established through the research, experiments and theoretical analysis, I have derived a set of design criteria that, if followed, will provide the basis for an expressively unique spatial visual performance instrument. The following five points are the main structural points for the overall instrument, each then broken down in successive sections, further elaborating on the specific needs of each part.

\section{Design Criteria for Spatial Visual Music Instrument}

The system should be considered as a loop; a loop between the performer (expression), the space (reality), and the content (experience). How the system functions provides the guidelines for interaction, where the system is set up for simplicity in use, yet remaining complex in possibility.

- Phenomenological interaction should engage the user movement in the entire zone of experience with the system, to maintain a tight relationship that is flexible and intuitive yet in control at all times

- The interface should be quickly apprehensible and provide immediately satisfying results by responding directly to real time performer input and actions

- The performance should be an immersive spatial experience, both virtually and physically, creating perceptive vulnerability through the augmented reality technique of projection mapping

- The exploration and navigation of the space should generate the visual content through illusively revealing and concealing the actual geometry of the space 


\section{System}

The system is the overall backbone that will hold all the individual components together.

- How the system works should provide the guidelines for the interaction

- The system should be set up for simplicity in use but remain complex in possibility

- The interaction with the system should be considered as a loop; a loop between the user, space and content. (Respectively; expression, reality and experience)

- The interaction between the user and the system should be intuitive in both ways

- The interaction should be approached phenomenological, where the interface is considered as immersive

- The system should be easy to understand yet mastery should remain illusive. The system should incorporate a learning and experience mechanism, where the final output should be a process of discovery

- The final output should have infinite variety, and have the ability for the performer to generate his/her own visual language

\section{Performance/Interaction}

The interaction side focuses on the connection between the performer and the system within the space

- A tight relationship between the performer and the system should be maintained for an intuitive performance. This performance should be based on improvisation, one that is flexible and adaptable, yet the user must remain in control at all times

- The phenomenological interaction should engage with human movement in the entire zone of experience

- The interaction should be based on gesture capturing techniques that provide the performer with visual cues and temporal marks of interaction that are recorded and played back

- The feedback loop of user interaction should not only be reflected but also refracted to provide the basis for content creation

- The interaction should be an all encompassing non-linear navigation of the space 


\section{Interface}

The interface deals with the more intimate connection between the user and system, and the details that the user is able to control

- The interface should respond directly to real time human movement, input and actions

- The performer should interpret the rules of the interface perceptually through experience, learning through discovery

- The interface should be quickly apprehensible and provide immediately evident results

- Within the interface there should be a balance between direct and indirect controls, which can be seen as individual and combination controls. The language of these controls should remain as an intuitive understanding to the performer

- The interface should address control of content creation and manipulation. These controls should be designed based on what is created and what is seen.

\section{Space}

The spatial points discuss the interaction of the physical and virtual spaces, in order to generate the content in the co-existence of these mentioned spaces

- The performance should be an immersive spatial visual experience, both virtually and physically, creating perceptive vulnerability through the augmented reality technique of projection mapping

- The merging of the physical and virtual space is an apparent loop of spaces where the virtual stands to supplement, mimic and deconstruct the physical

- The composition of spaces should be considered as a medium in itself, where the spatial feedback can then be identified as 'space medium'

- Interacting with the 'space medium' is based on the deconstruction of reality, revealing and concealing the actual physical geometry of the space. The actual geometry should be navigated as an exploration of space, where the manipulation of the 'space medium' sculpts the illusion. 


\section{Visual Music}

The visual music section discusses the actual content creation, how it is generated, and how it is performed to the audience

- The exploration and navigation of the space should generate the visual content through the spatial illusion of revealing and concealing the actual geometry

- The 'space medium' generated from the spatial feedback loop, should be considered as the visual medium. The space medium will generate it's own principles and behaviours that should provide an unlimited possibility for compositions through ever changing user inputs. Visual inputs will vary in two formats, from pure light and shadow to a more graphical approach to the geometry

- The compositions should be evident in real time, with the possibility to combine temporal and looped compositions. It is the deconstruction of the compositional loops that becomes the performance. 


\section{Summary}

\section{Summary}

These criteria discussed form the detailed framework for building and designing an intuitive visual performance instrument through the use of spatial illusion. They are merely guidelines, yet derived from research that puts a theoretical outline towards the actual physical outcome, and therefore provide enough substance to create such an instrument.

In the following chapter, the designed system is discussed through a diagrammatic essay, and finally the performance of the instrument is elaborated on to demonstrate the effective nature of the system in creating a visual performance through spatial illusion. 
Chapter 6

\section{Final \\ System \\ Design}




\section{Final System Design}

The following Chapter will present a break down of the final system design in individual sections, covering the overall system setup, the interaction setup and the interface layout. For this design several decisions have been made to facilitate the design in its purest form. Firstly the space has been minimalised to demonstrate better its layout and performability, although it is possible for it to take on almost any format. The space has been reduced down to one cube in the middle of two planes. This provides the user with enough physical 3-Dimensionality to demonstrate the principles established within the research. Secondly the actual interface has been achieved through deconstructing two Nintendo-Wii remotes controllers to achieve the spatial navigation as desired. Each of these decisions will be discussed in detail in the following sections. 

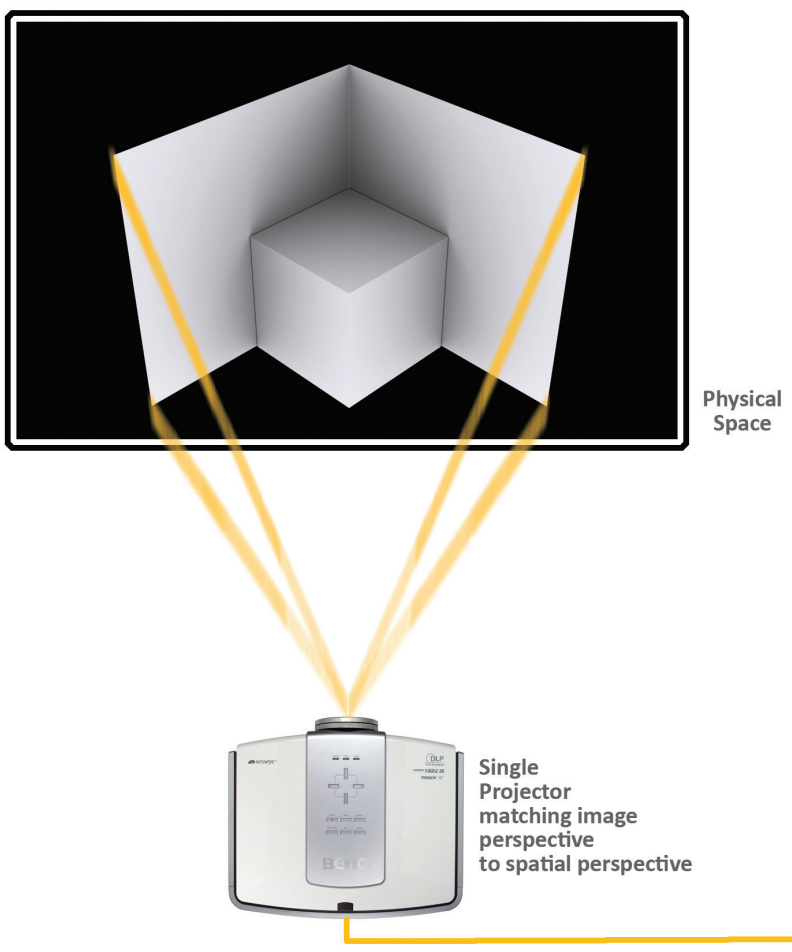

mage is used as texture on each plane

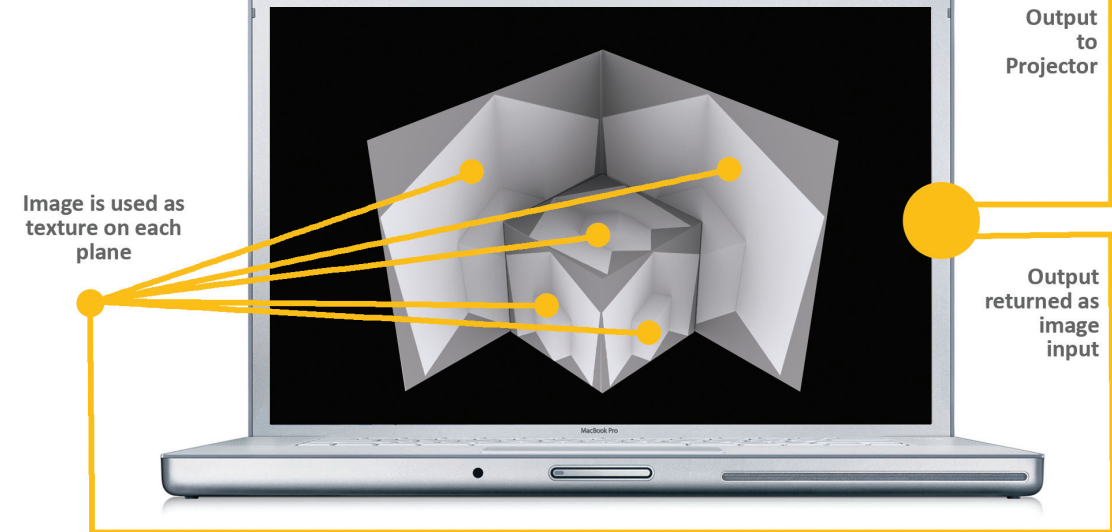




\section{Performance System}

This covers the overall performative Projection Mapping layout of the design system. The system is broken down into two sections, the physical space and the virtual space. The physical space is set up as desired, and then replicated within the virtual space. All the virtual planes are matched in perspective and projected back onto the physical space, creating this Projection Mapping surface.

Secondly that same digital image is returned to the virtual space as a texture, mapped onto each surface within the design, which in turn is projected back out onto the physical surfaces. This immediately creates a digital feedback loop, where the process is now repeated onto every surface, physical and virtual, through this internal repetition. This process has now created the space-medium, as mentioned in the research. This space medium can now become the main medium to be performed with in the system. 
This set up determines how the user interacts with the system. Bodily navigation is the main concept for interaction, as established in the research, therefore the system is dedicated to making this possible. This project interprets bodily navigation as physical pointing to geometry in the performance space, guiding the work through the space. This is achieved through using the motion of the hands of the performer, as similarly discussed in the Theremin experiment. Hand-eye co-ordination gives the system a strong basis for the design, which avoids arbitrary gestures from the performer. It is separated into two parts of interaction, one focussed on the physical through initiation and the second on the virtual through navigation.

The initiation process is the first step evident in the performance. In this step the user controls a dot that can be used to reveal and conceal the physical space.

Figure 133

Diagram outlining the elements within the interaction system

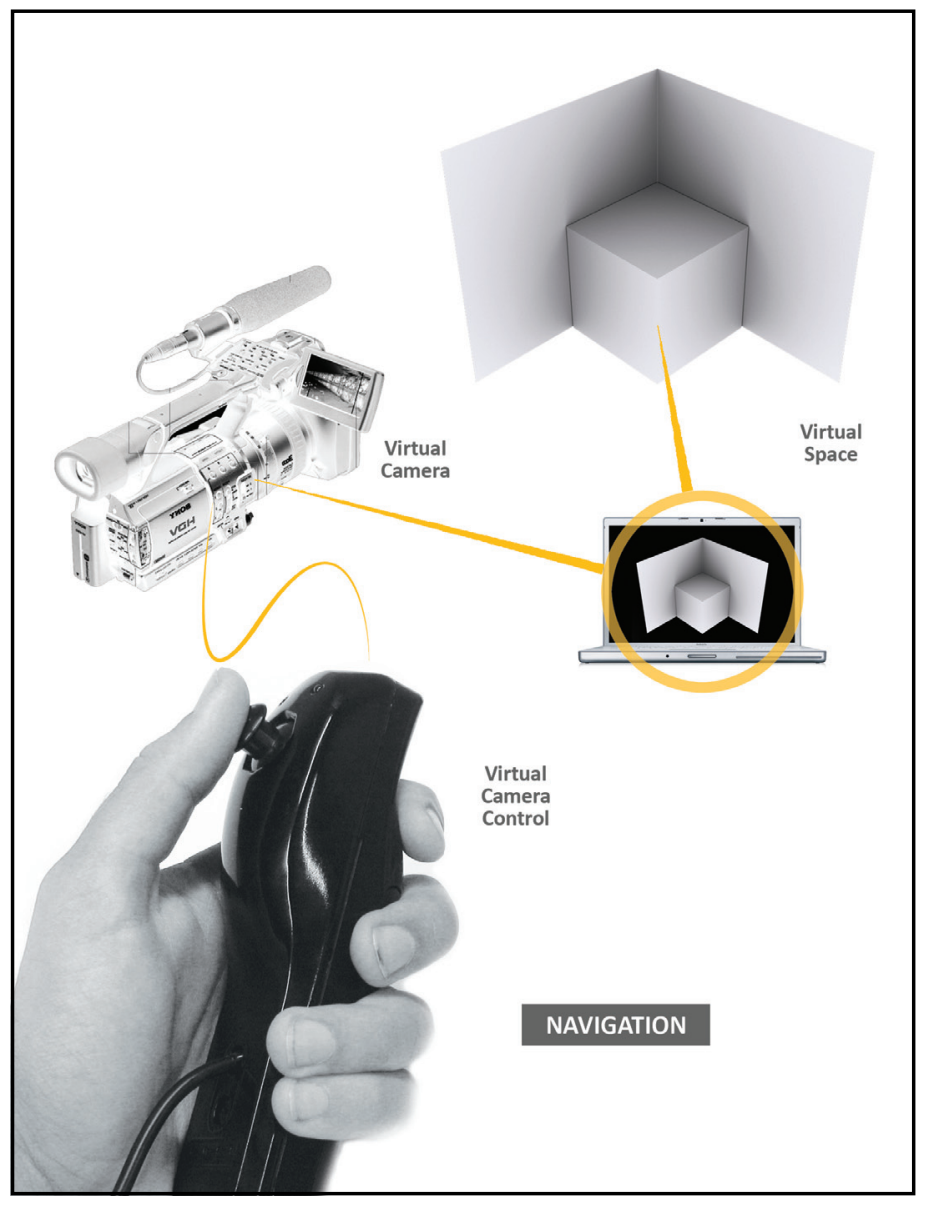


This process of revealing and concealing can be likened to the use of a torch in the dark to highlight certain geometries. The user also has the ability to change the actual shape of the dot through scaling in the $x$ and $y$ directions. This function can adjust the dot in scale or change it into a line in any direction. The user now has a completely malleable initiation platform from which to create animation through the process of revealing and concealing.

The navigation function is the second step in the process of interaction. The feedback signal, as mentioned in the previous section, that returns the virtual back as a feedback loop onto the surfaces as a mapped texture is provided by a "virtual camera." This "camera" can now become the second controller by being completely free to manipulate. The feedback is now the graphical performance element, in the process of revealing and concealing space.

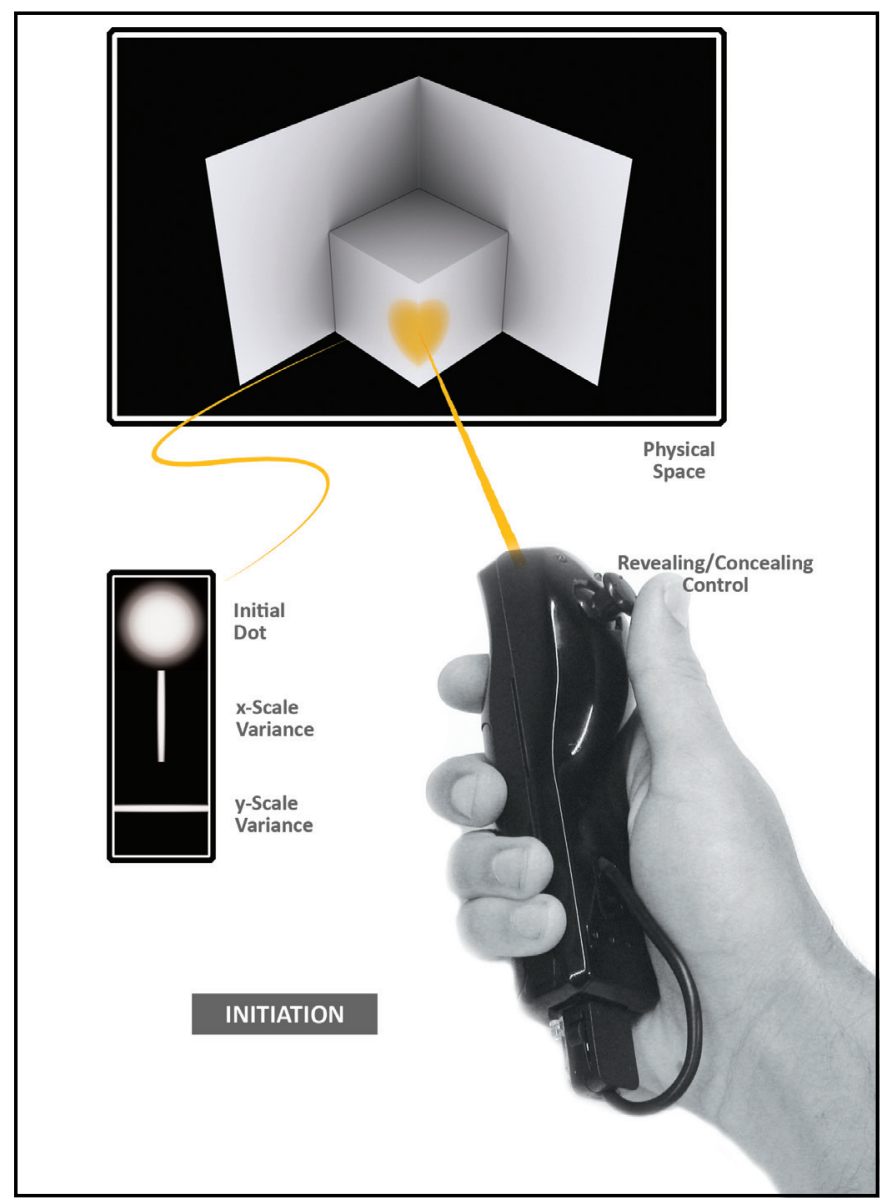

Figure 134

Diagram outlining the elements within the interaction system 


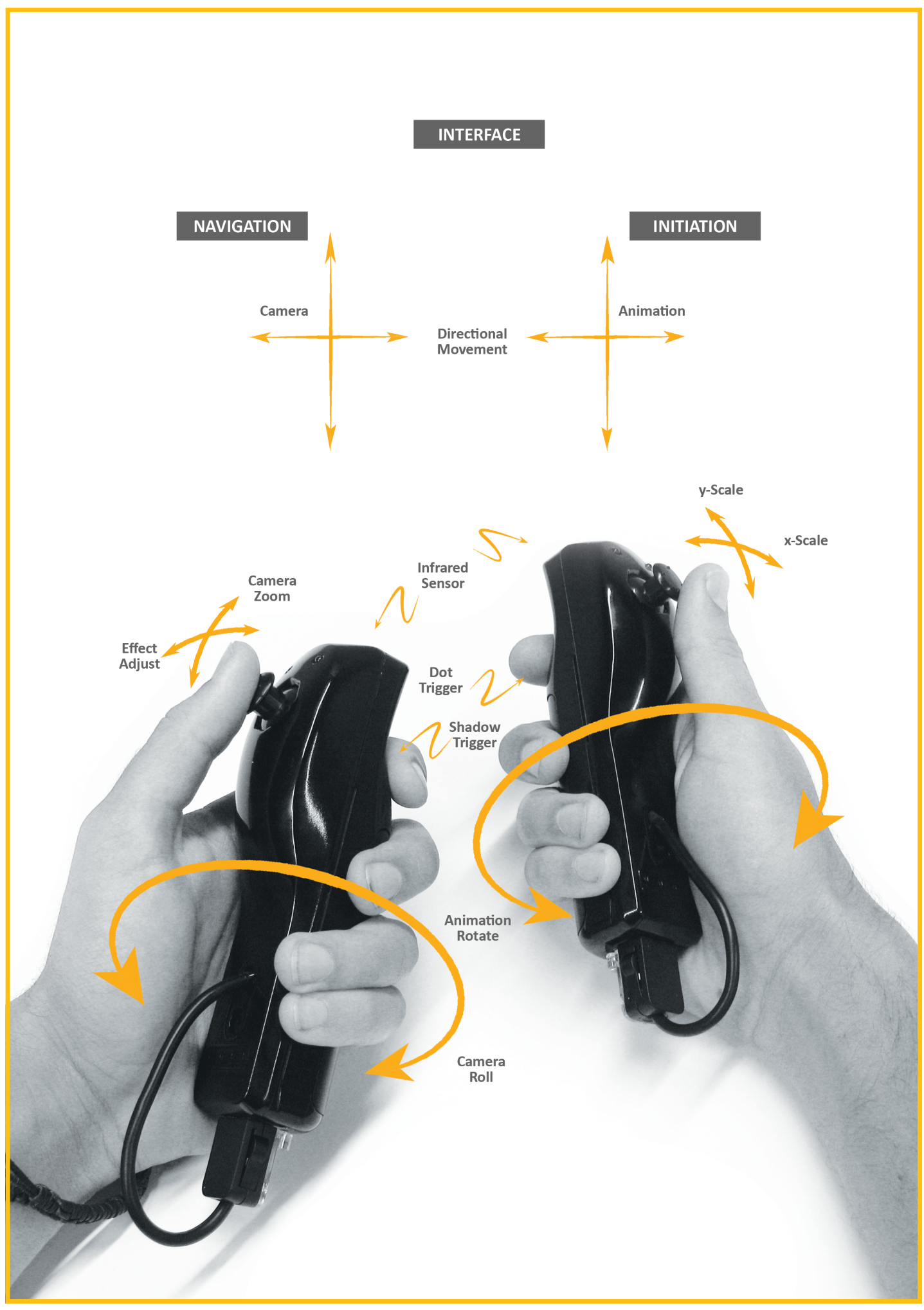




\section{Interface Layout}

Each section of the interaction relates to a specific component on the controller. In this case I have used, broken down and modified two sets of Nintendo Wii Remote Controller replicas, as they provide the right spatial navigation functionalities needed for the interaction. Firstly the accelerometer data is used as spatial positioning, movement and roll, and secondly the infrared triangulation determines the directional movement of the controller. Extra animation functionalities that do not relate to movement are controlled through an externally attached joystick. The combination of spatial navigation techniques provides the perfect resolve in line with the intuitive bodily navigation established in the research analysis.

Within the initiation interface, the main component is the directional movement of the graphical spot on the physical surface, where the directional movement is controlled through the infrared triangulation. From there the animation picks up and responds to the roll of the controller in turn providing the roll of the animation component. The shape and scale of the animation is lastly controlled through the externally attached joystick.

The navigation, controlling the "virtual camera" operates in a very similar way. Again, the actual directional movement of the camera is controlled through the infrared triangulation from the point of view of the controller. Secondly, the roll of the camera responds to the roll data received from the accelerometer, and lastly the camera zoom is controlled through the joystick. I have included another function in joystick to aid the performability of the feedback, although it is not vital to the project. This can be an effect control, which can be manipulated in the same way, the simplest of which can merely be a colour scale adjust. 

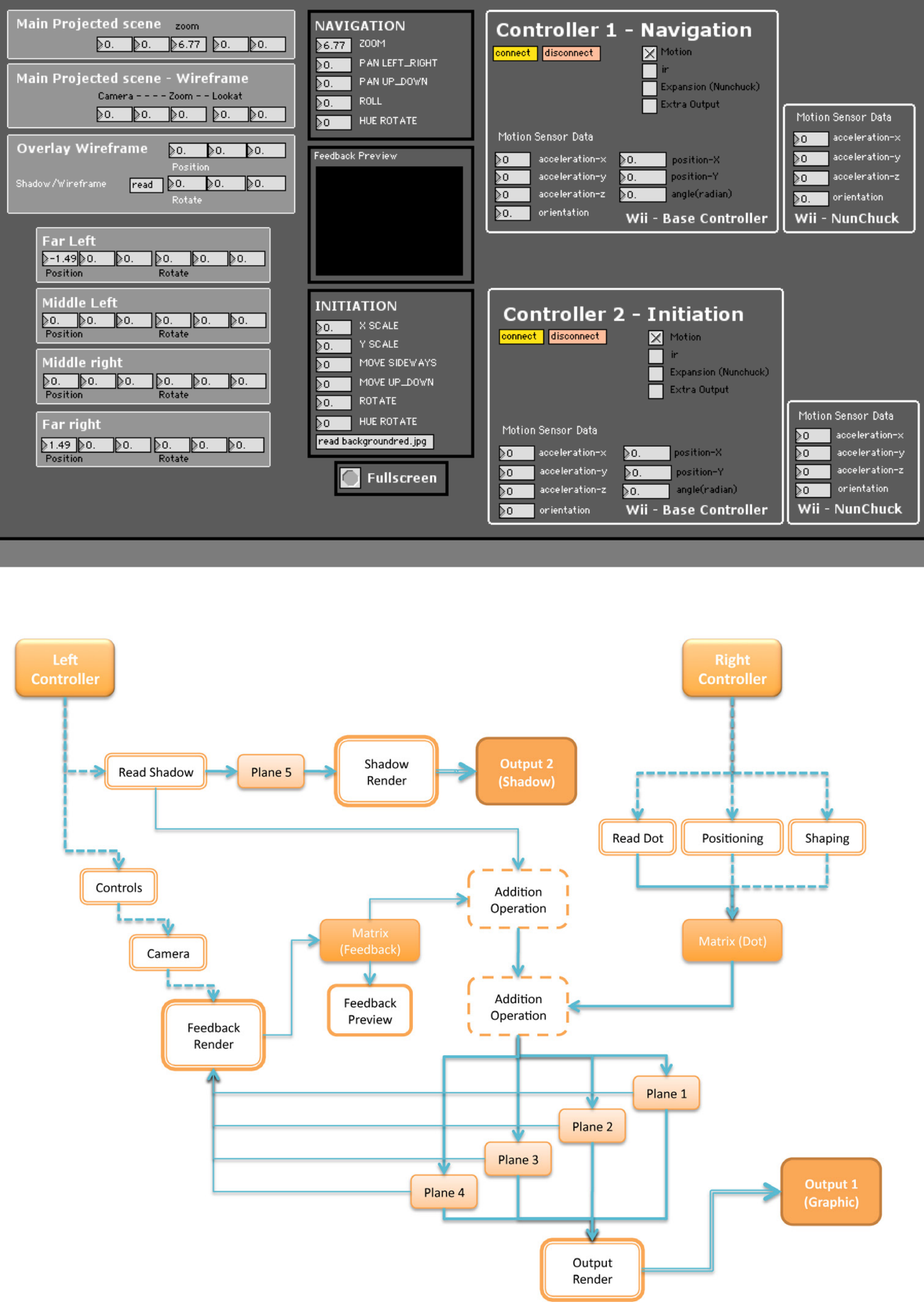


\section{Software Setup}

MaxMSP/Jitter, the visual programming software provided through Cycling '74 (http://cycling74.com/), has been used to create the digital environment for the performance. The patch written can be described in four parts:

- 3-Dimensional Scene Setup

- Animation Control Display

- Controller Activation

- Dual Projector Outputs

Apart from the initial setup, the performer does not need to deal with a computer screen, only with the modified remote controls. It exists purely to interpret the information received from the controller system and to generate the visual content. The system can be performed in two modes; as a graphical visual music and as a realistic output dubbed 'wireframe.' The 'wireframe' mode is so named as the renderings are based on the actual wireframe geometry of the given scene. These two modes of output can also be played in unison for an even more complex performance.

\section{Scene setup}

The main scene (graphical output) is constructed through individual planes within the OpenGL environment. These planes are arranged into a format that mimics the physical scene that is to be projected on. Each plane can be added, subtracted, positioned and rotated. The virtual scene can also be adjusted through the main virtual 'camera' as to match the projection angle and therefore the physical perspective.

The wireframe scene is less complex in terms of setting up but requires more animation work prior to using the system. Here only one plane exists that runs an animation that is to be projected out over the entire physical scene. This virtual wireframe scene can also be adjusted in terms of projection angles and camera perspective.

\section{Animation Control Display}

This section acts merely as a visual display representing the activities during the performance. The navigation section relays all the information received from the Navigation controller. This controls the virtual camera that displays the feedback
Figure 136

Image showing the MaxMSP/Jitter patch that was built for the system. Alongside is a diagram outlining how the patch works. 
signal. This feedback signal can be viewed in the 'Feedback Preview' window merely as an indicator of orientation. The initiation section again only relays information received from the Initiation controller, providing numerical feedback for the initial orientation of the animation.

\section{Controller Activation}

The controller activation is divided into two identical parts, one for each controller. The only interaction here is connecting the controllers to the software. All paths are then connected automatically through the controller display and on to the animations and projections.

\section{Dual Projector Outputs}

The output has been split over two projectors for two reasons. Firstly due to the processing limitations of MaxMSP/Jitter, which runs a lot smoother and efficient without having to compile two outputs into one. Secondly, the system is set up to be performed on two modes, therefore each mode has it's own output. These projector outputs are from the same projection angle and thereby work through the same projection mapping technique.

Figure 136 provides a summary of the whole MaxMSP/Jitter patch. This is purely to demonstrate a simplified version of the complexity within the patch to provide a clear understanding of how the software system functions. The right controller, or animation controller, provides three points of data; activating the dot, positioning the dot and shaping the dot. This information is passed onto each plane and rendered out. To create the feedback loop these planes are also rendered back as a whole into a matrix video stream which is then added back into the original animation. The left controller, Virtual Camera, controls the feedback video stream, which produces the spatial feedback animation on each plane. The left controller also activates the 'wireframe' mode, which can be passed back onto the planes individually to recreate the feedback technique for the wireframe mode. 


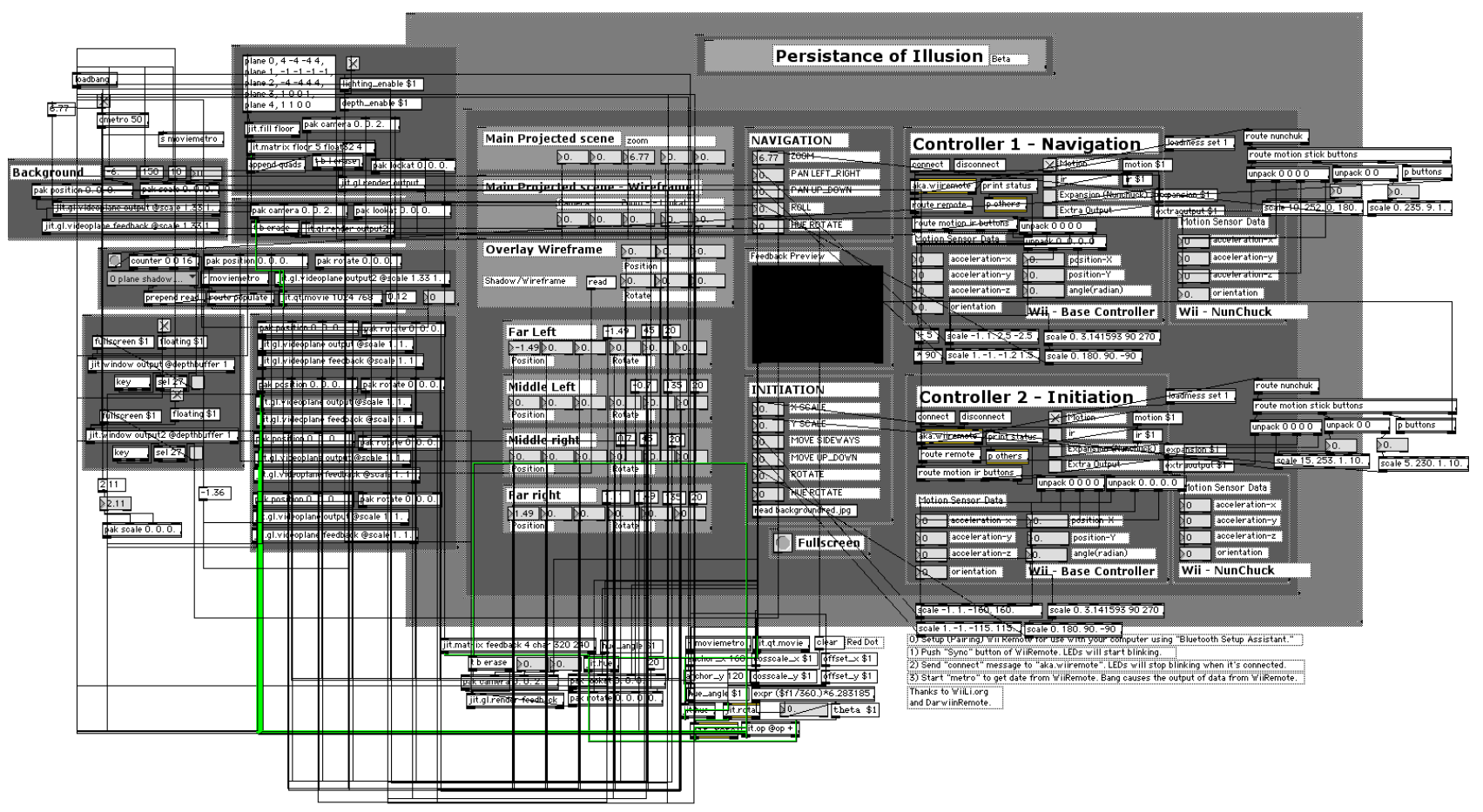

Figure 137

Image showing the internal mess of theMaxMSP/Jitter patch that was built for the system. 

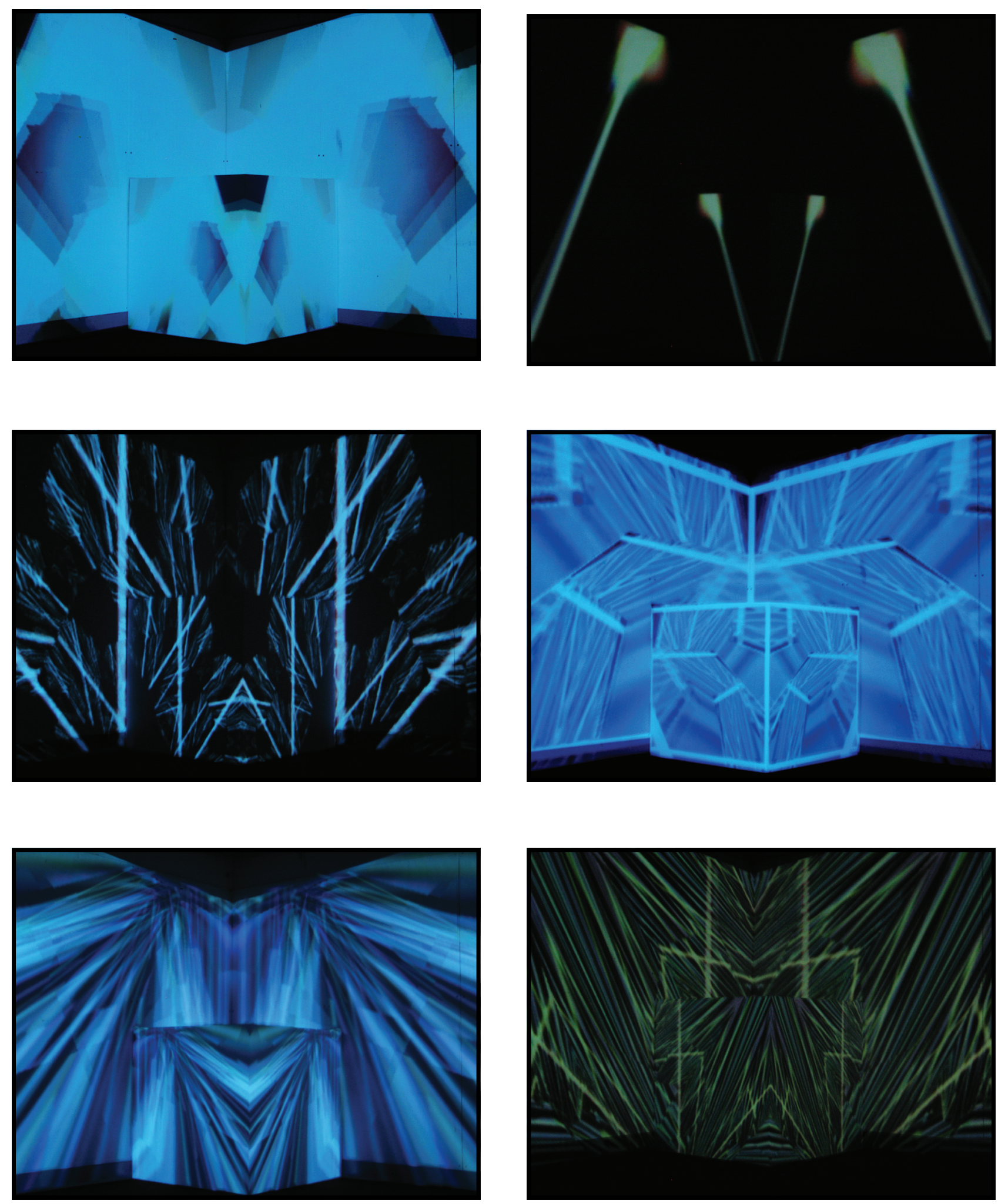


\section{Performance Documentation}

The following section is an image documentation of the visual imagery created by the system. As it is difficult to describe the success of a moving medium through still image or text, a DVD is provided with a recording, illustrating how the system interaction works, and what affect the controllers and the performer has on the visual imagery.

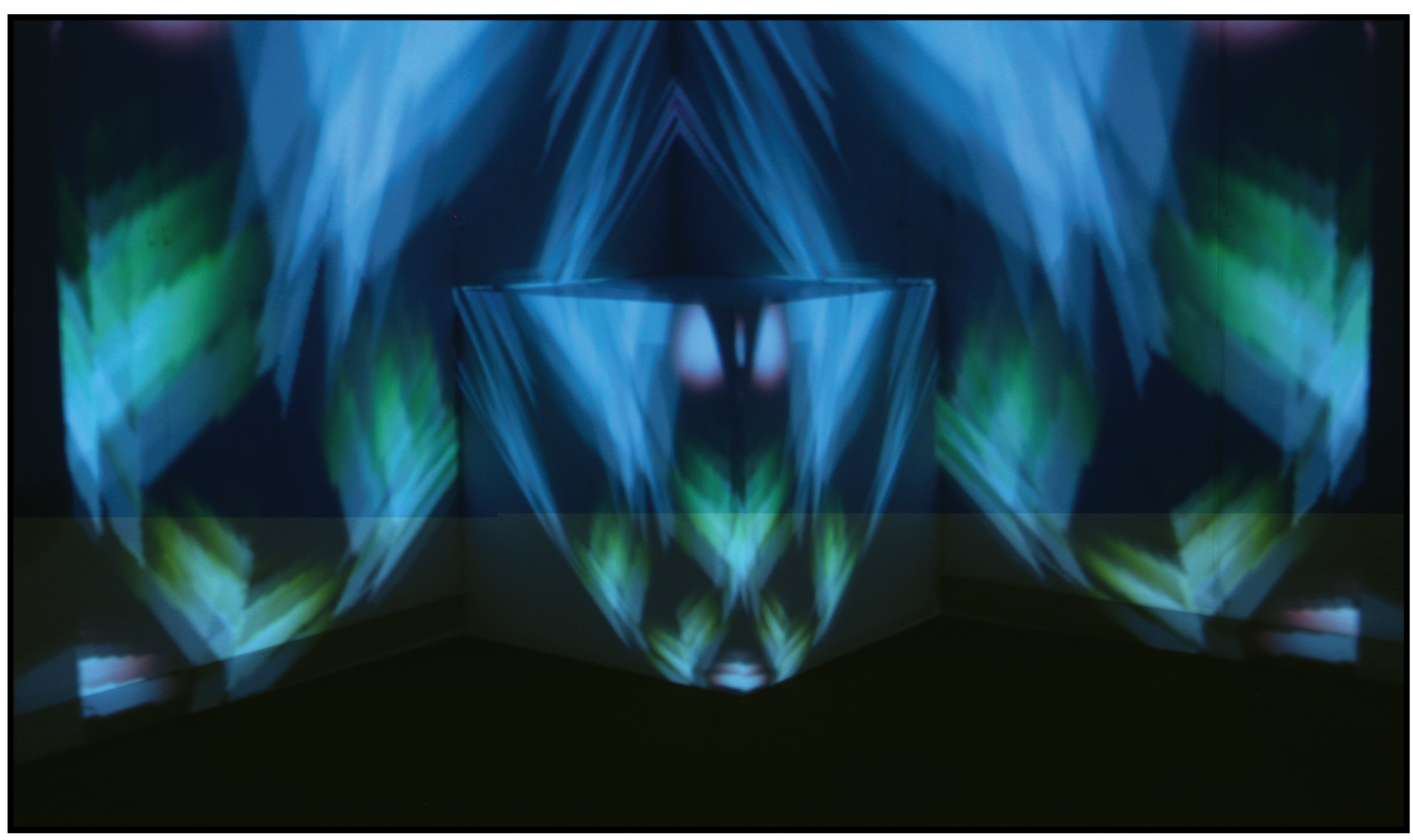



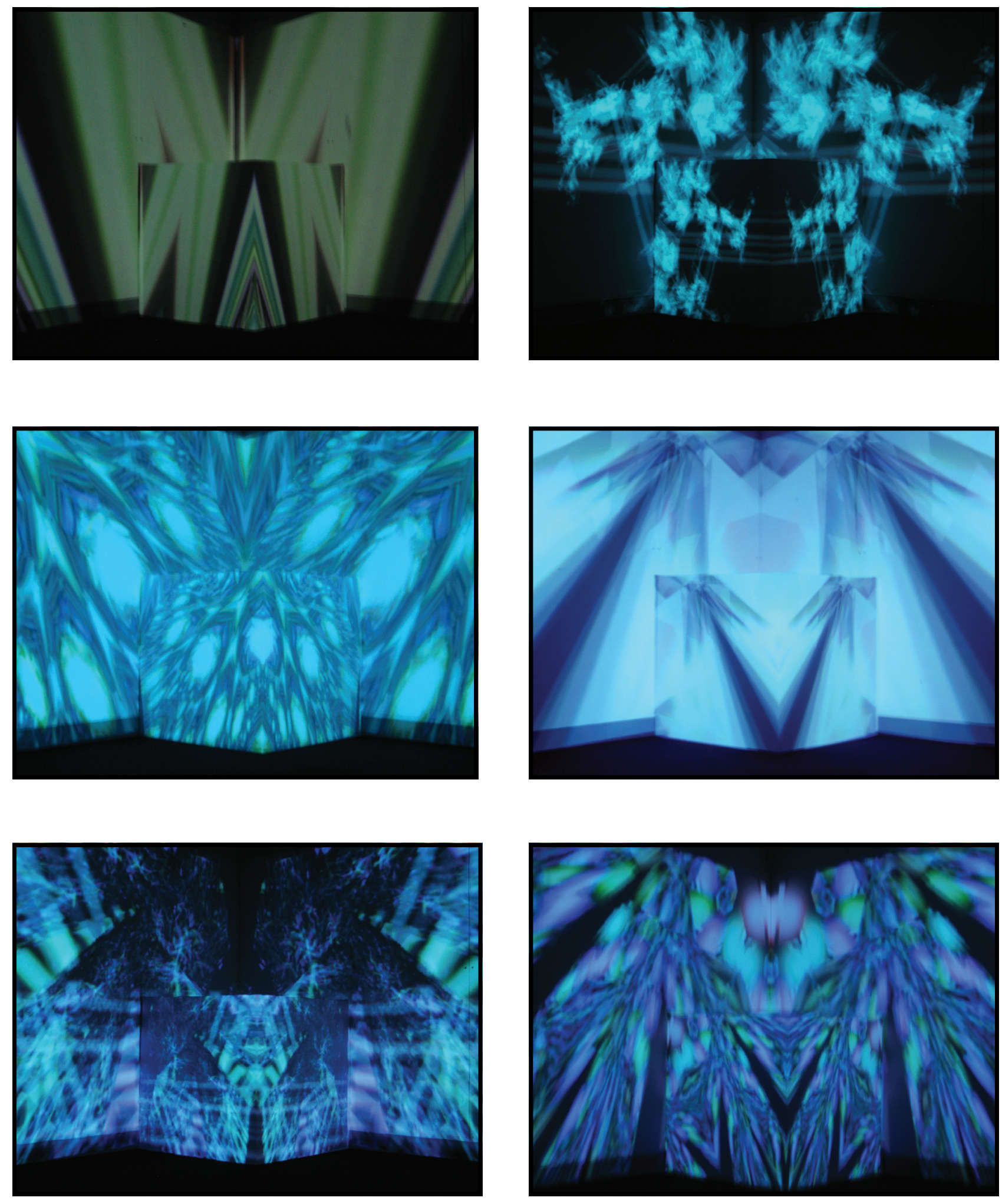

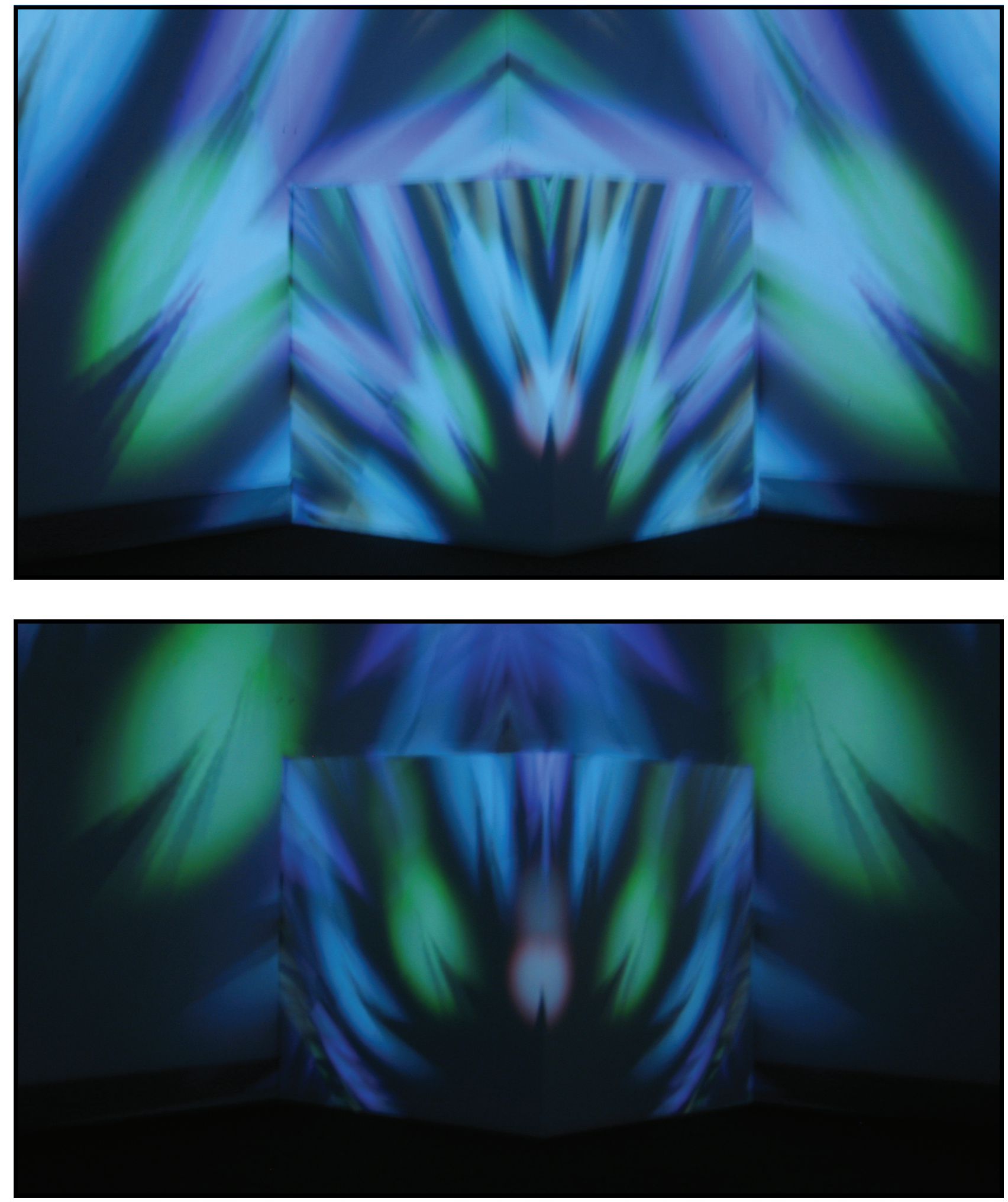

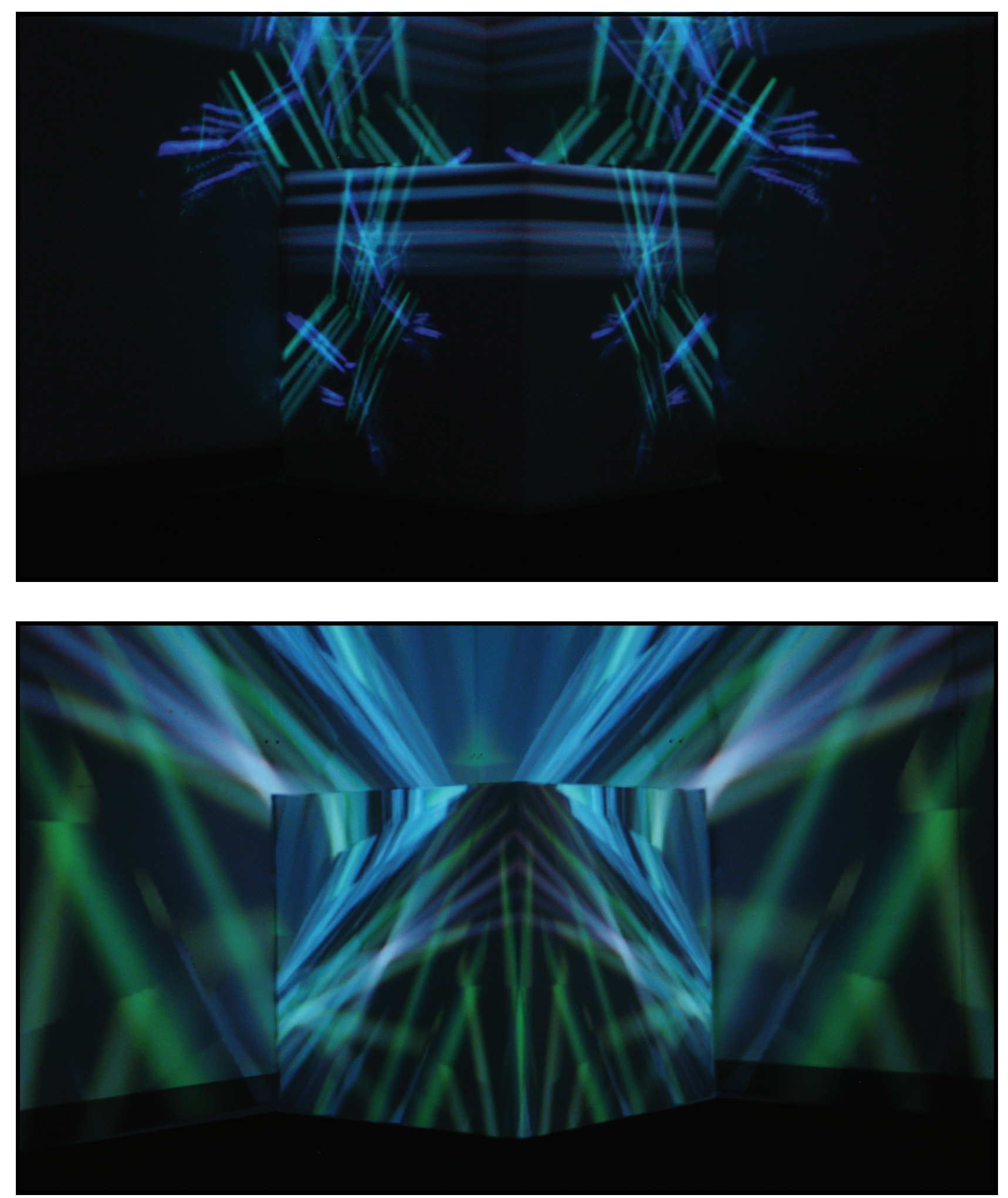

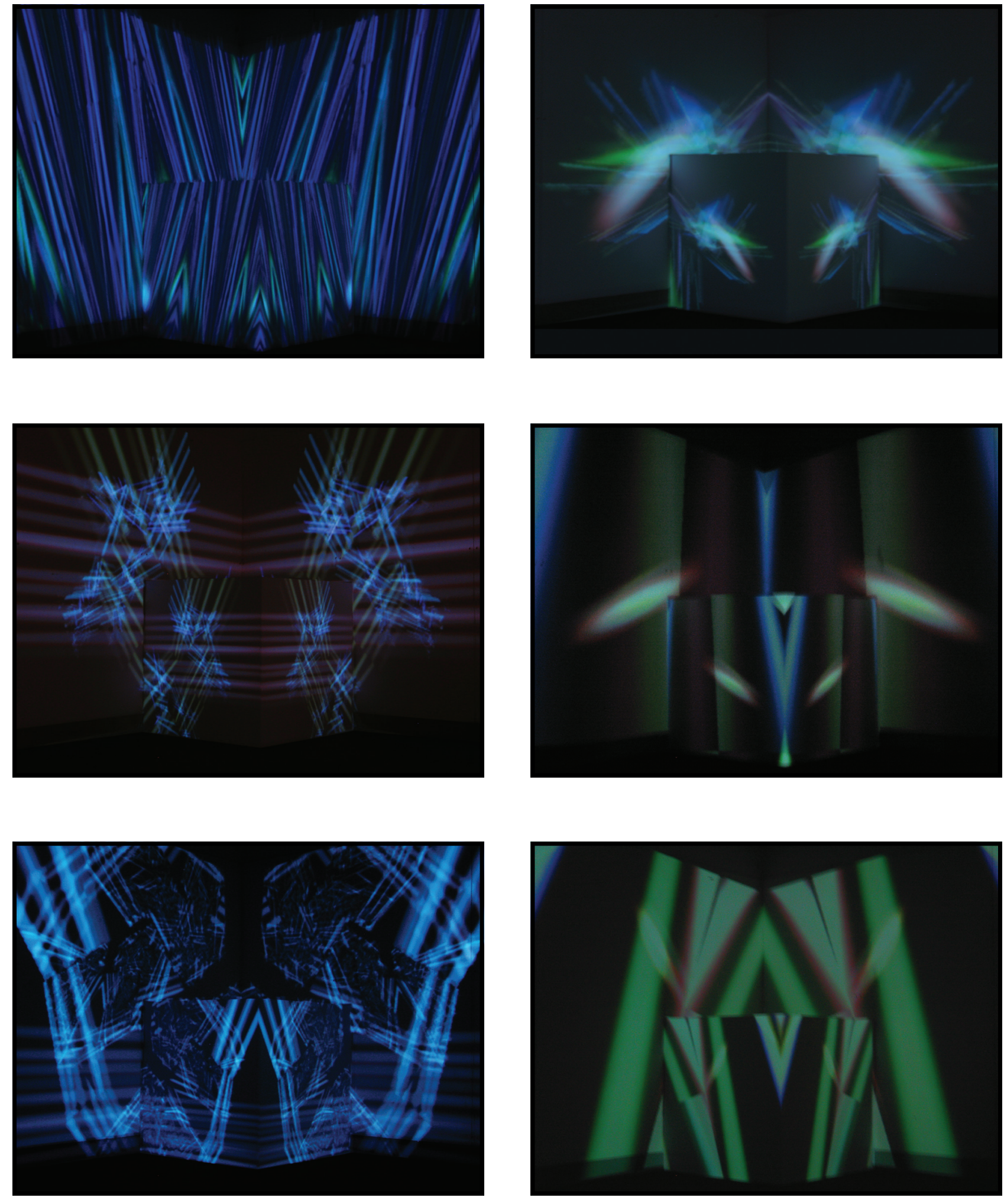

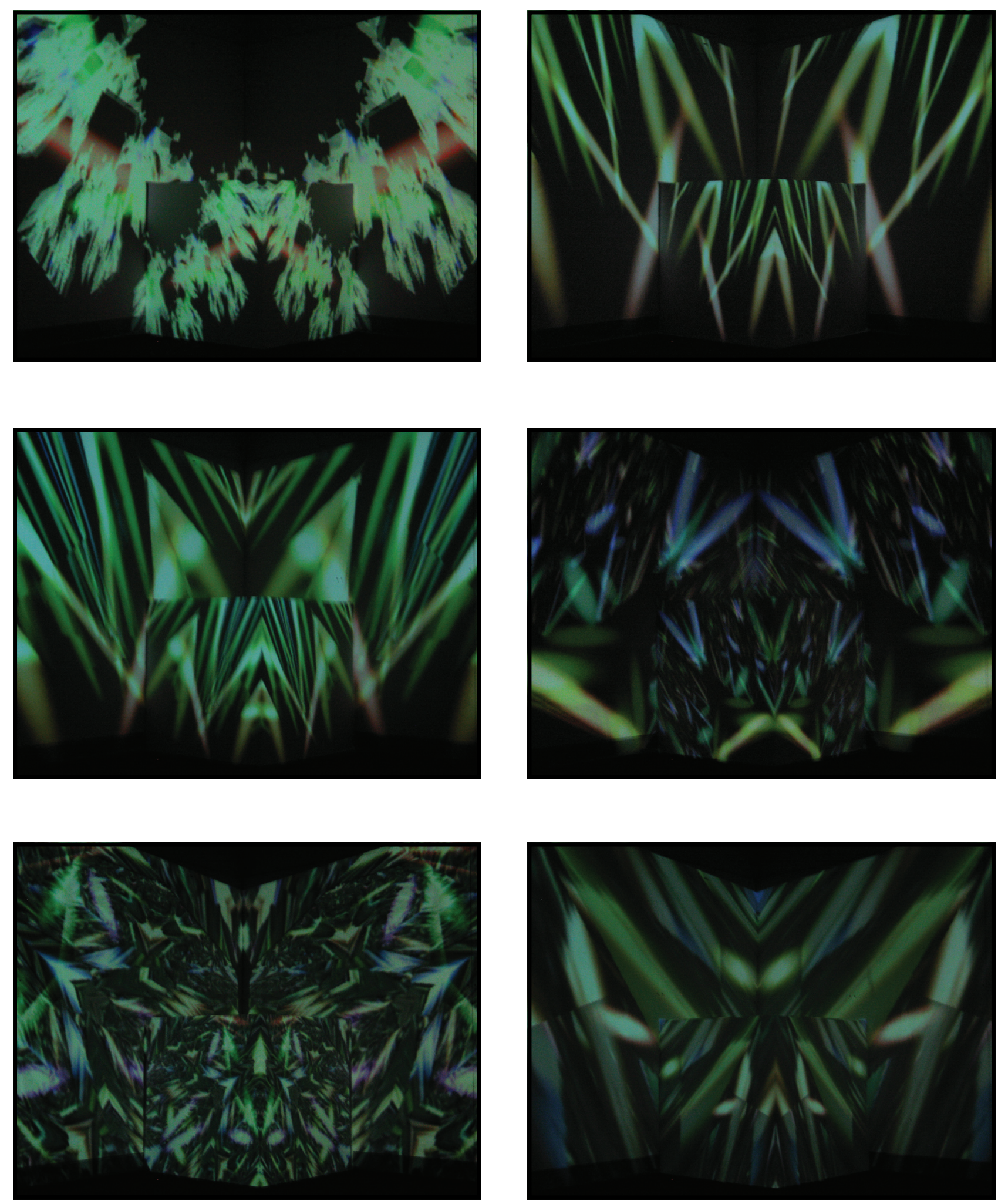

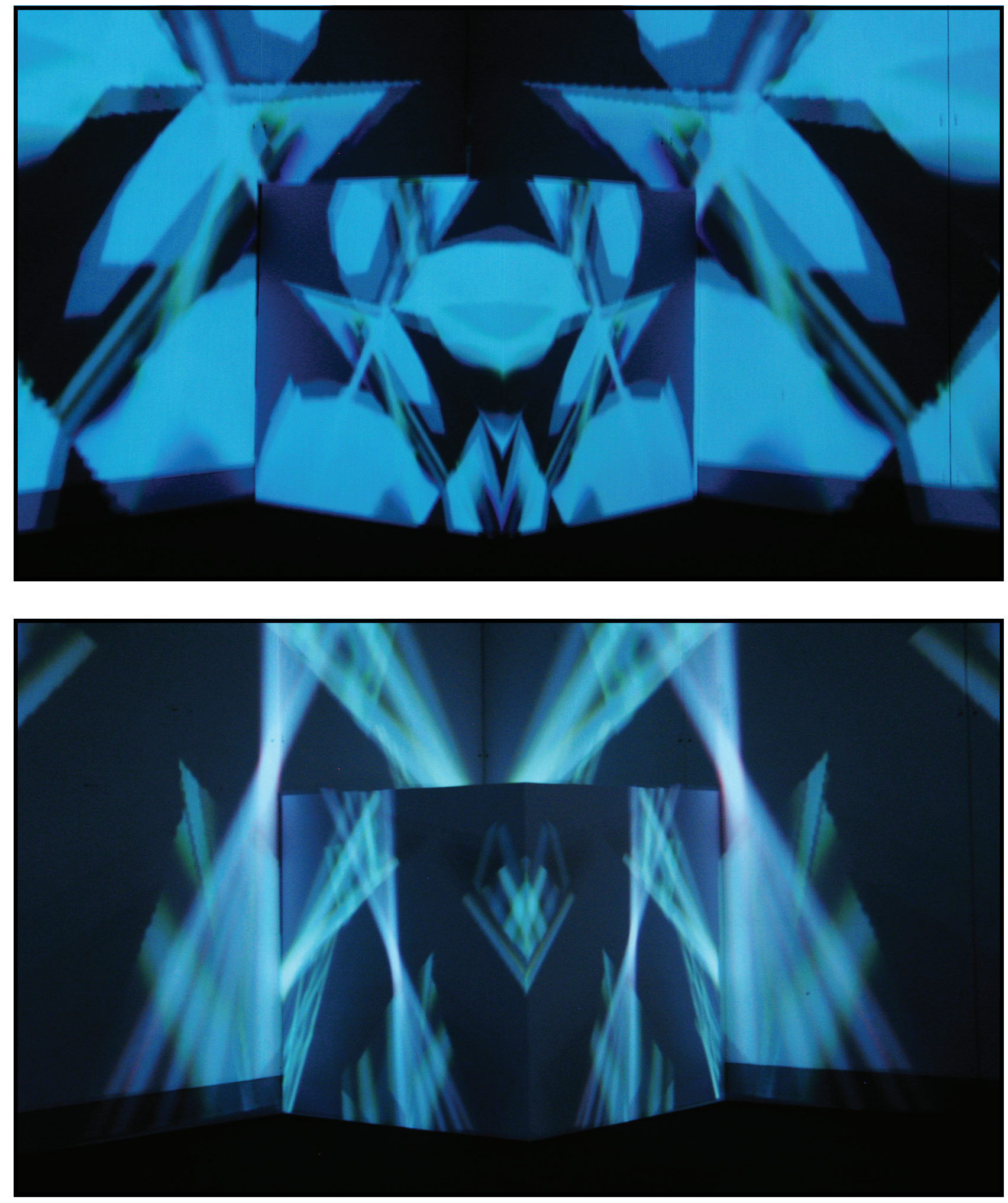
Chapter 7

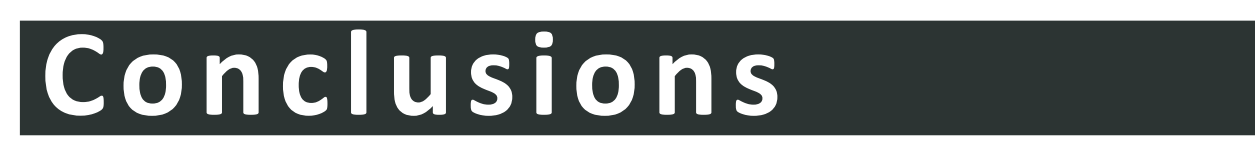




\section{Conclusions}

\section{Summary}

The contextual analysis acts as an analytical background study, but rather than providing a timeline, it lines up the thesis with a contextual relevance. Historical and contemporary investigations both provide the research methodology with valuable ideologies in the search for the ideal visual performance situation. Many contemporary visual music systems have done much to advance the knowledge gained from the pre-cinema era, yet lack the same innovation in interaction within the systems. Contemporary systems, although quite sophisticated in visual output, are still very much attached to the generic mouse and keyboard combination, or stylus and sketchpad inputs, which seem to have taken a step backward from the more intuitive approaches set out by the likes of Oscar Fischinger's Lumigraph. This thesis therefore takes this computer sophistication in output and matches it more to the sort of interaction evident in the early precinema instrumentation.

Throughout the experiments the goal was to demonstrate some of the principles evident throughout the initial research, with a special focus on projection onto irregular surfaces. Possibly the most interesting way of addressing the notions of visual imagery on a 3-Dimensional canvas rather than a flat sheet, is the use of illusion, which has provided the research with the key concepts of revealing and concealing space. Through experimenting with these ideas the research presented a resolution not only for the content creation but also provided a basis for creating the interaction. This combination of navigable interaction and content creation become the main underlying concept throughout the research. This finding opened up the investigation to include theory on how this combination of interaction and content creation actually function and co-exist.

The screen, as we currently understand it, is identified as a limitation to a visual performance, especially as at this point a much larger portion of the space is included in the projection. The importance and relevance of Projection Mapping is discussed as a much more contemporary and relevant means of addressing a visual performance. Projection Mapping can cover any space the artist chooses, and therefore provides the artist with a much richer canvas from which to operate. Projection Mapping itself is a combination of virtual and physical space, identical, complimentary and deceptive. This combination of spaces, which the thesis addresses as the space-medium, really becomes a medium in itself. This 
space-medium is evaluated as a standalone medium that can be manipulated as a whole, and not one of individual parts that are individually manipulated. This medium manipulation stands to become a new form of visualization and not just representation. An extension from performative visual art yet with much more depth as it extends of the screen and into the physical world.

Identifying the loop on three levels; the dialogue between the physical and virtual space, the space medium as content generator and the interaction between the user and the system, provides the research with a strong basis from which to develop the performance system. Although extremely critical, the loop concept has endured very superficial evaluations within the VJ and media art community. Extending the theory of the loop, pushing its analysis into all areas of the live visual artists' performance, proved that the loop in fact constitutes an entire visual performance and visual performance instruments in all their detail.

The success of the investigations allowed for a set of design criteria to be created for a visual performance system. The criteria are an extremely crucial part of the analysis as it provides the very detailed framework for the final design. In summary, the criteria identifies that the interaction should engage gestural user movement in the entire zone of experience to maintain a tight relationship with the system, that the interface should be quickly apprehensible, that the performance should be an immersive experience utilizing the projection mapping technique, and finally that exploration and navigation of the space should provide the basis for content creation and performance interaction. The criteria are set out in such a way that the final designed output can vary exceptionally in results, yet they are strong enough to retain the core idea of a visual performance system, so that any designed output will remain successful in its purpose. The criteria identified cover every aspect of the visual performance system and is therefore followed closely to be able to judge the project as a success. The designed system, which covers the overall system functionality, interactivity, interface and content creation, proves most successful when linked closely to the design criteria established. The intuitive means of controlling the visuals brings on the image or metaphor of the Illusionist, a visual artist that becomes part of the performance composition with a real "performer presence." Finally the validity of the of the system concept is demonstrated through a performance, revealing itself not merely as an investigation, but proof that such a system is a successful move towards a visual performance instrument. 


\section{Discussion}

The intention of this thesis is to provide a framework for visual performance systems, based on intuitive bodily interaction within the performance environment. This interaction introduces the idea of using the actual spatial identities of the performance environment as the visual content creator. This system as a whole I have termed an instrument, as the term instrument clearly identifies the principles needed for a successful performance system. As identified the term instrument encompasses the ideals of simplicity in use yet remaining complex and illusive in possibility. The research therefore through this process identified the specific criteria needed for a successful visual performance instrument.

There are a few concepts addressed in this thesis that stand out significantly in relation to other research that has been carried out in this field of study. These include the unique use of space rather than software environments as the main driving component, the system of intuitive interaction, the ability to generate new original content at every performance, and the idea of valid "performer presence."

The use of spatial notions in this thesis as the main driving force has evolved from the use of Projection Mapping as a practical methodology. Currently media art is not pushing Projection Mapping as an interactive means of performance, but merely a 3-dimensional projection canvas. This thesis has taken a fresh look at Projection Mapping and evolved it to become the main guidance throughout the design of the visual performance instrument. It has moved the starting point of this system design away from software, thereby forming the basis for content creation and user interaction.

The research also significantly breaks away from a traditional or standard look at computer interfacing. The interface encompasses a spatial bodily response that has no functions or menus to select from, but purely reacts fluidly to the movements and guidance of the performer. The most interesting part of the visual content creation during the performance is that it will never be the same at separate performances. This is a concept quite familiar to visual artists, as the aim is provide a new experience at each opportunity. As this performance involves the actual physical space that is being projected on, each context and 
physical layout will provide varying visual results. An interesting point here, as it means that although the basics of the instrument can be mastered, each performance will provide new challenges, in effect creating an instrument that is infinitely variable in its outcomes. This type of infinitely variable performance will require an artist to have extensive experience and skill in creating and performing these visual compositions. The visual performer's skill basis now moves from 'software operator' to 'real-time visual compositionist,' a very independent form of visual art. The artist is now able to step into a role that has real performer presence, and is no longer bound to reside behind a laptop computer.

\section{Future Directions}

Presented is a very analytical point of view in regards to the overall research. This will provide a very strong basis for future development in regards to projection mapping instruments. This research has taken the attention away from using the computer as the starting point in the development, providing a methodology in which the computer only exists as a tool within the system rather than being the instrument itself. I can see this as a step towards a new type of visual media performer; a projection mapping performer. More intensive investigation into this field can truly push the performance of visual music to a more pronounced level, if not a commercial level, which would be an ultimate goal.

This concept then of creating a live visual performance whilst separate from the physical computer does deserve a lot more attention in media design to a point where, again, these concepts can be pushed towards a commercial level. I personally would have liked to investigate the actual design of the physical components within this thesis, however I have found that a really strong theoretical understanding of the system is in fact much more vital. As with the component design, and the aforementioned ideas of a new type of performer and interaction, I think these topics could encompass an entire thesis individually in their own right.

It is my hope that this research will provide a fresh look at projection mapping interaction, especially on a performance level, where bodily navigation is engaged in interaction through multiple points, movements and emotions. 


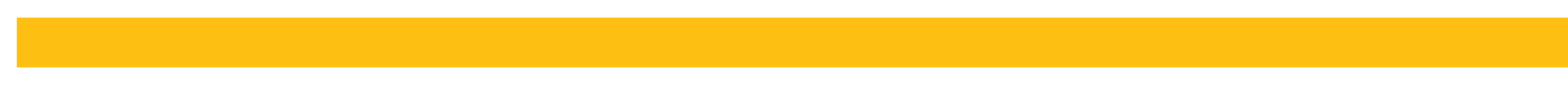




\section{Bibliography}

\section{Books}

Allain, Paul, \& Harvie, Jen,. (2006). Routledge Companion to Theatre and Performance, The. New York, NY: Routledge.

Baecker, Ronald M., \& Buxton, William, \& Grudin, Jonathan (Eds.). (1995). Readings in Human-Computer Interaction: Toward the Year 2000. San Francisco, CA: Morgan Kaufmann.

Barfield, Lon. (2004). Design for New Media: Interaction design for multimedia and the web. Harlow, England: Pearson Education Limited.

Csikszentmihalyi, Mihaly. (1991). Flow: The Psycology of Optimal Experience. Harper Collins.

Dabrowski, Magdalena. (1997). Kandinsky: Compositions. New York: Harry N. Adams.

Dawes, Brendan. (2007). Analog In, Digital Out: Brendan Dawes on Interaction Design. Berkeley, CA: New Riders.

Gerstner, Karl. (1986). The Forms of Color: The Interaction of Visual Elements. Cambridge, MA: MIT Press.

Greenewalt, Mary Hallock . (1946). Nourathar: The Fine Art of Light Color Playing. Philadelphia: Westbrook.

Jaeger, Timothy. (2005). Live Cinema Unraveled. Published by writer.

Kandinsky, Wassily. (1947). Concerning the Spirituality in Art. New York: George Wittenborn, Inc.

Kandinsky, Wassily. (1994). Point and Line to Plane. In Kenneth C. Lindsay \& Peter Vergo (Eds.). Kandinsky: Complete Writings on Art. New York, NY: Da Capo Press.

Klee, Paul. (1961). The Thinking Eye. New York, NY: George Wittenborn.

Klein, Adrien Bernard. (1927). Colour-Music: The Art of Light. London: Crosby, Lockwood \& Son.

Krueger, Myron W. (1991). Artificial Reality II. Reading, Massachusetts: Addison-Wesley

Lister, Martin, \& Dovey, Jon, \& Giddings, Seth, \& Grant, lain, \& Kelly, Kieran. (2003). New Media: A Critical Introduction. New York, NY: Routledge.

Manovich, Lev. (2001). The Language of New Media. Cambridge, Massachusetts: The MIT Press.

Marks, Lawrence E. (1978). The Unity of Senses / Interrelationships Among the Modalities. New York: New York Academic Press

Mattis, Olivia, \& Brougher, Kerry, \& Strick, Jeremy, \& Wiseman, Ari, \& Zilczer, Judith. (2005). In Hirshhorn Museum and Sculpture Garden, \& Smithsonian Institution, \& The Museum of Contemporary Art. Visual Music: Synaesthesia in Art and Music Since 1900. New York, NY: Thames \& Hudson Inc. 
Morgenstern, Sam. (1956). Composers on Music: An Anthology of Composer's Writings from Palestrina to Copland translated from Sounds and Colors in Essai sur l'origine des langues (1753). New York, NY: Pantheon Books 54-55

Moritz, William. (1993). "The Dream of Color Music," in The Spiritual in Art: Abstract Painting 1890-1985 Maurice Tuchman, (Ed.). New York, NY: Abbeville.

Natanson, Maurice. (1973). Edmund Husserl: Philosopher of Infinite Tasks. United States: Northwestern University Press.

O’Sullivan, Dan, \& Igoe, Tom. (2004). Physical Computing. United States of America: Stacey L. Hiquet

Rimington, Alexander Wallace. (1911). Colour-Music: The Art of Mobile Colour. New York, NY: Frederick A. Stokes Company.

Rokeby, David. (1995). Transforming Mirrors: Subjectivity and Control in Interactive Media. In Simon Penny (Ed.). Critical Issues in Electronic Media. New York: State University of New York Press.

Rokeby, David. (1996). Transforming Mirrors: Subjectivity and Control in Interactive Media. Albany, NY: Suny Press.

Turner, Victor W., \& Bruner, Edward M. (Eds.). (1986). The Anthropology of Experience. United States of America: University of Illinois Press.

Youngblood, Gene. (1970). Expanded Cinema. New York: E.P. Dutton.

Zajonc, Arthur. (1995). Catching the Light - The Entwined History of Light and Mind. Oxford University Press

\section{Journals and Websites}

AntiVJ, (2009). Personal Website. Retrieved from http://www.antivj.com/

Baranoff-Rossiné, Dmitri. (1997). The Piano Optophonique of Wladimir Baranoff- Rossiné. Retrieved from http://perso. club-internet.fr/dbr/piano.htm

Barsamian, Gregory. (2009). Personal Website. Retrieved from http://www.gregorybarsamian.com/media/start.html Bishop, Bainbridge. (2007). The Harmony of Light. Retrieved from http://rhythmiclight.com/books/ HarmonyOfLight. pdf

Collopy, Fred. (1999). Imagers and Lumia. Retrieved from http://imagers.cwru.edu/index.html

Collopy, Fred. (2003). Designing an Instrument to Perform Abstract Animation in Real-Time. Retrieved from http:// www.kether.com/liveCinema/SFPCS/Collopy-designing.html 
Dekker, Annet. (2003). Synaesthetic performance in the club scene. Retrieved from http://www.cosignconference.org/ conference/2003/papers/dekker_cosign_2003.pdf

DeWitt, Tom. (1987). Visual music: Searching for an aesthetic. Leonardo, 20, 115-122

Dove, Arthur. (1929). Notes by Arthur G. Dove. In Dove Exhibition. New York, NY: The Intimate Gallery

Interval Research Corporation. (1997, October). Towards Dynamic Abstraction (Internal document).

Palo Alto, CA: Scott Snibbe and Golan Levin.

IRSA. (1987). Maud Miles 1914. Artibus et historiae, 16. University of Michigan

Levin, Golan. (2000). Painterly Interfaces for Audiovisual Performance. Massachusetts Institute of Technology.

Levin, Golan. (2006). The Table is the Score: An Augmented Reality Interface for Real-Time, Tangible, Spec-

trographic Performance. Retrieved from http://www.flong.com/texts/publications/pub_icmc06/levin_

scrapple_20060320_1200dpi.pdf

Levin, Golan, \& Lieberman, Zachary. (2005). Sounds from Shapes: Audiovisual Performance with Hand Silhouette Contours in The Manual Input Sessions. Retrieved from

http://www.flong.com/texts/publications/pub_nime05_mis/NIME_2005c_MIS.pdf

Lew, Michael. (2004). Live Cinema: Designing an Instrument for Cinema Editing as a live performance Retrieved from http://alumni.media.mit.edu/ /ew/research/livecinema/Live\%20Cinema\%20NIME\%202004.pdf

Maclean, Karon, \& Snibbe, Scott, \& Levin, Golan. (2000, April). Tagged Handles: Merging Discrete and Continuous Control. Proceedings from ACM SIGCHI 2000. Retrieved from http://www.media.mit.edu/ golan/handles/ index.html

Maeda, John. (1995). Digital Expression: A Framework for Artistic Creation of Forms On and Using the Computer. (Ph.D Thesis). University of Tsukuba, Japan

Makela, Mia. (2006). Live Cinema: Language and Elements. Helsinki University of Art and Design.

Peacock, Kenneth. (1988). Instruments to perform color-music: Two centuries of technological experimentation. Leonardo, 21, 397-406

Popper, Frank. (1968). Origins and development of kinetic art. New York Graphic Society, 24, 156-172.

Rokeby, David. (1986-1990). Very Nervous System. Retrieved from http://homepage.mac.com/davidrokeby/vns.html

Snibbe, Scott, (1998-2010). Personal Website. Retrieved from http://www.snibbe.com/

Snibbe, Scott, \& Levin, Golan. (2000, June). Interactive Dynamic Abstraction. Proceedings of the First Annual Conference on Non-Photorealistic Animation and Rendering. Annecy, France. Retrieved from http://www.flong.com/texts/ publications/pub_npar00/dynamicNPAR.pdf 
Snibbe, Scott. \& Schopf, Christine (Ed.). (1996). The Motion Phone. Proceedings of Ars Electronica '96. Retrieved from http://kultur.aec.at/lab/futureweb/english/prix/prix/1996E96azl-motion.html

VVVV. (2009). How to project on 3D Geometry. Retrieved from

http://vvvv.org/tiki-index.php?page=How+To+Project+On+3D+Geometry

Weiss, Frieder. (2006). I, Myself and Me Again. Retrieved from

http://www.frieder-weiss.de/works/all/Laborgras.php

Wilfred, Thomas (1948, December). Composing in the art of Lumia. The Journal of Aesthetics of Art Criticism 7, 2. 89

William Moritz. (1997, April). The dream of color music, and machines that made it possible. Animation World Magazine, Issue 2.1. Retrieved from http://www.awn.com/mag/issue2.1/articles/moritz2.1.html 


\section{Image source for Figures}

1. Melissa Cowan Photography. (2009). http://www.melissacowanphotos.co.nz

2. Melissa Cowan Photography. (2009). http://www.melissacowanphotos.co.nz

3. Melissa Cowan Photography. (2009). http://www.melissacowanphotos.co.nz

4. Melissa Cowan Photography. (2009). http://www.melissacowanphotos.co.nz

5. $\quad$ Fred Collopy. (2004). http://www.RhythmicLight.com

6. Retrieved from http://www.wassilykandinsky.net/

7. Retrieved from http://www.humanitiesweb.org/human.php?s=g\&p=c\&a=p\&ID=997

8. Mattis, Olivia, \& Brougher, Kerry, \& Strick, Jeremy, \& Wiseman, Ari, \& Zilczer, Judith. (2005). In Hirshhorn Museum and Sculpture Garden, \& Smithsonian Institution, \& The Museum of Contemporary Art. Visual Music: Synaesthesia in Art and Music Since 1900. New York, NY: Thames \& Hudson Inc.

9. Len Lye Foundation, New Zealand

10. Len Lye Foundation, New Zealand

11. Len Lye Foundation, New Zealand

12. Published in the Scientific American, June 1930

13. Mattis, Olivia, \& Brougher, Kerry, \& Strick, Jeremy, \& Wiseman, Ari, \& Zilczer, Judith. (2005). In Hirshhorn Museum and Sculpture Garden, \& Smithsonian Institution, \& The Museum of Contemporary Art. Visual Music: Synaesthesia in Art and Music Since 1900. New York, NY: Thames \& Hudson Inc.

14. Mattis, Olivia, \& Brougher, Kerry, \& Strick, Jeremy, \& Wiseman, Ari, \& Zilczer, Judith. (2005). In Hirshhorn Museum and Sculpture Garden, \& Smithsonian Institution, \& The Museum of Contemporary Art. Visual Music: Synaesthesia in Art and Music Since 1900. New York, NY: Thames \& Hudson Inc.

15. Fischinger, Oscar. Retrieved from http://www.snibbe.com/

16. Levin, Golan. (2000). Retrieved from http://www.flong.com

17. Snibbe, Scott. (1998-2010). Personal Website. Retrieved from http://www.snibbe.com/

18. Snibbe, Scott. (2003). Personal Website. Retrieved from http://www.snibbe.com/

19. Levin, Golan. (2007). Retrieved from http://www.flong.com

20. Rokeby, David. (1986-1990). Retrieved from http://homepage.mac.com/davidrokeby/vns.html

21. Weiss, Frieder. (2006). Retrieved from http://www.frieder-weiss.de/works/all/Laborgras.php

22. Weiss, Frieder. (2006). Retrieved from http://www.frieder-weiss.de/works/all/Laborgras.php

23. Weiss, Frieder. (2006). Retrieved from http://www.frieder-weiss.de/works/all/Laborgras.php

24. Weiss, Frieder. (2006). Retrieved from http://www.frieder-weiss.de/works/all/Laborgras.php

25. Weiss, Frieder. (2006). Retrieved from http://www.frieder-weiss.de/works/all/Laborgras.php

26. AntiVJ. (2009). Personal Website. Retrieved from http://www.antivj.com/

27. VVVV. (2009). How to project on 3D Geometry. Retrieved from http://vvvv.org/tiki-index.php?page=How+To+Project+On+3D+Geometry

28. VVVV. (2009). How to project on 3D Geometry. Retrieved from http://vvvv.org/tiki-index.php?page=How+To+Project+On+3D+Geometry

29. VVVV. (2009). How to project on 3D Geometry. Retrieved from http://vvvv.org/tiki-index.php?page=How+To+Project+On+3D+Geometry

30. $-126 . \quad$ Provided by the Author of this Thesis

127. - 130. AntiVJ, (2009). Personal Website. Retrieved from http://www.antivj.com/

131. - 137. Provided by the Author of this Thesis

138. $-168 . \quad$ Provided by the Author of this Thesis 


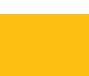


Appendix 1

\section{Visual Music}

All the images provided in Appendix 1 are sourced from

Mattis, Olivia, \& Brougher, Kerry, \& Strick, Jeremy, \& Wiseman, Ari, \& Zilczer, Judith. (2005). In Hirshhorn Museum and Sculpture Garden, \& Smithsonian Institution, \& The Museum of Contemporary Art. Visual Music: Synaesthesia in

Art and Music Since 1900. New York, NY: Thames \& Hudson Inc. and do not belong to the Author of this Thesis 


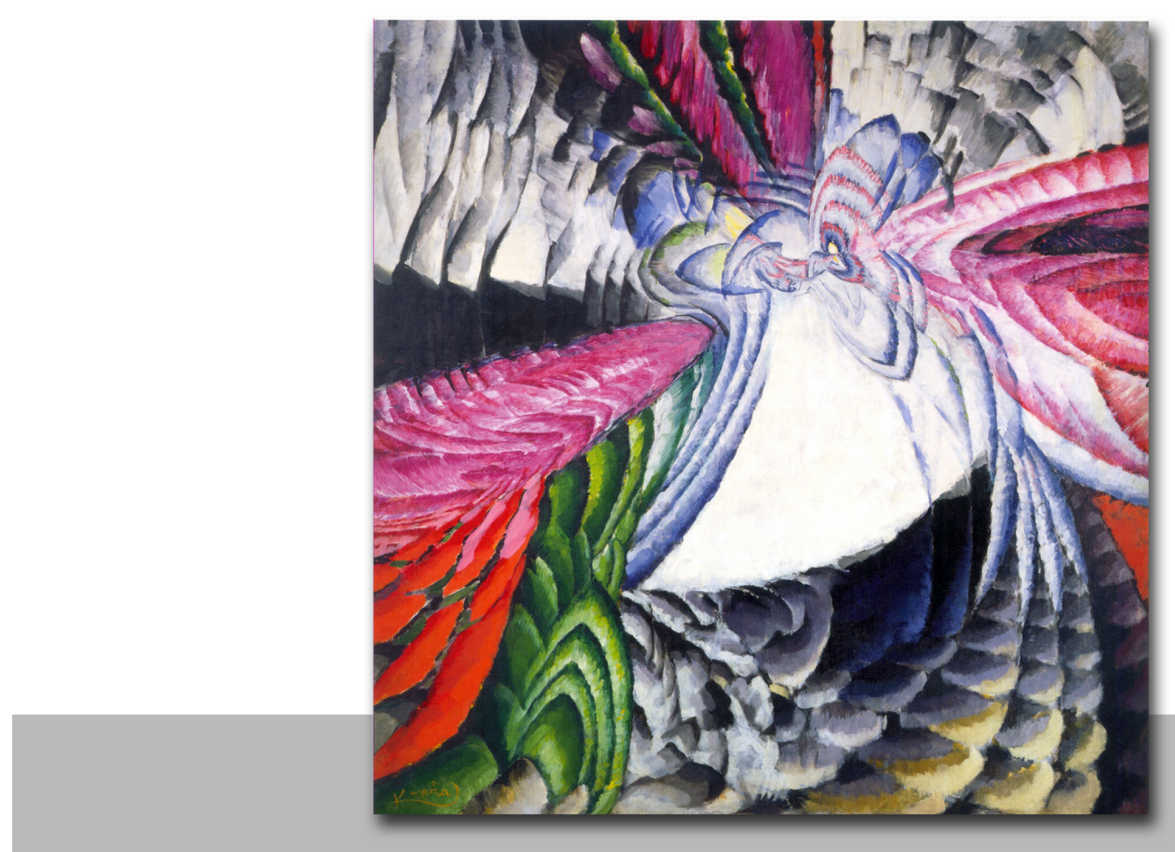

1912_13 Frantisek Kupka - Organization of Graphic Motifis II

1912 Frantisek Kupka - Disks of Newton

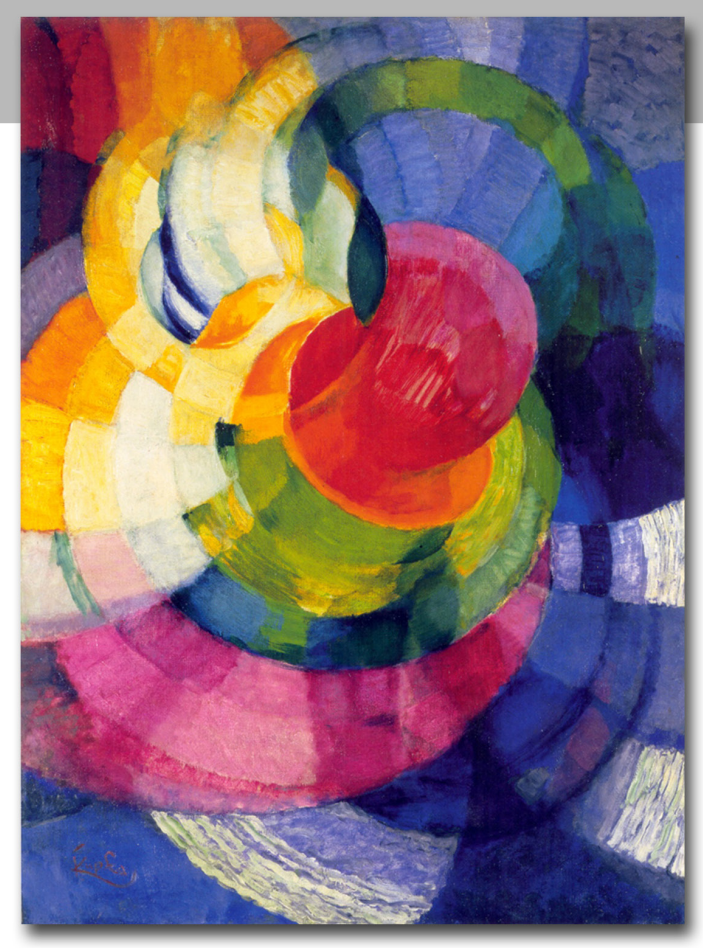

1913 Leopold Survage - Rythme Colore

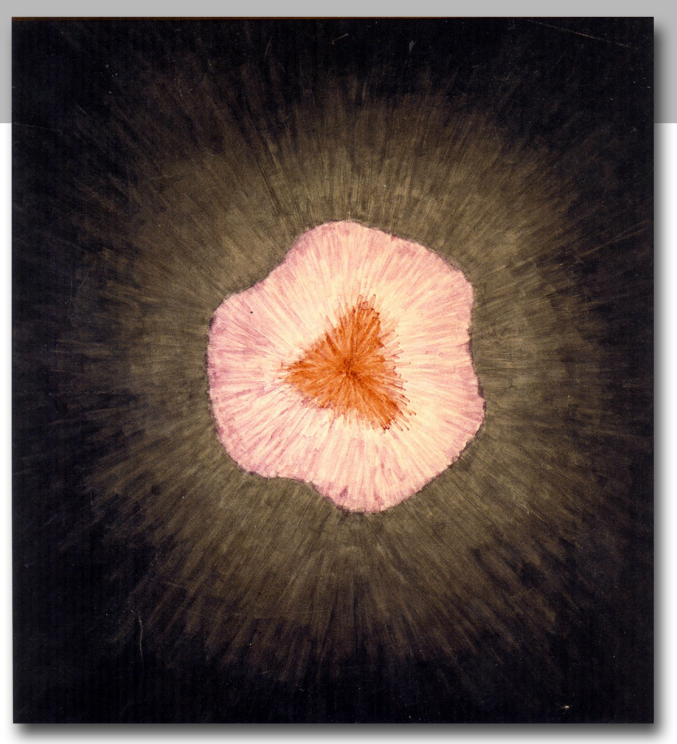




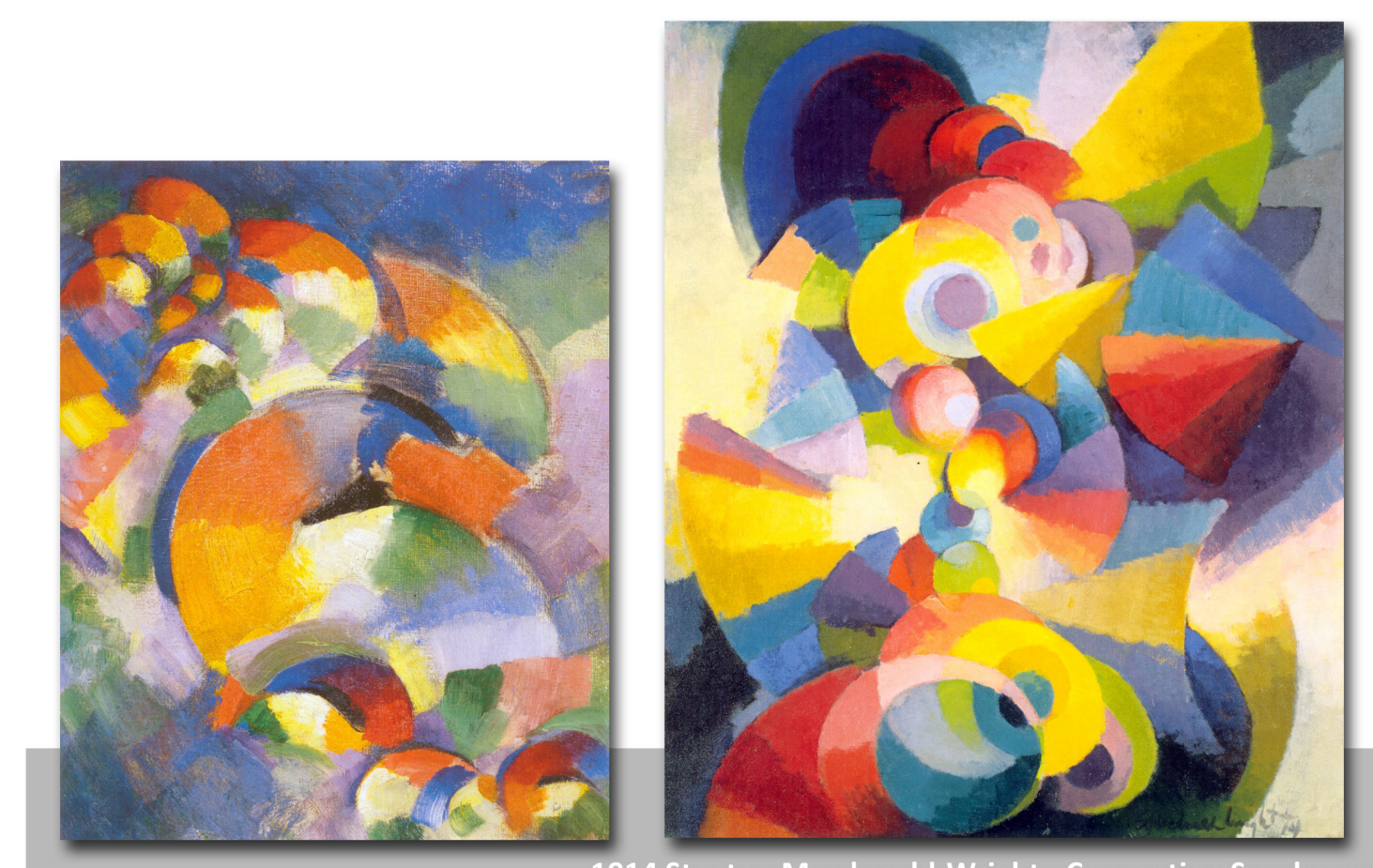

1914 Morgan Russell - Cosmic Synchromy

1914 Stanton Macdonald-Wright - Conception Synchromy

1914 Wassily Kadinsky - Fuga

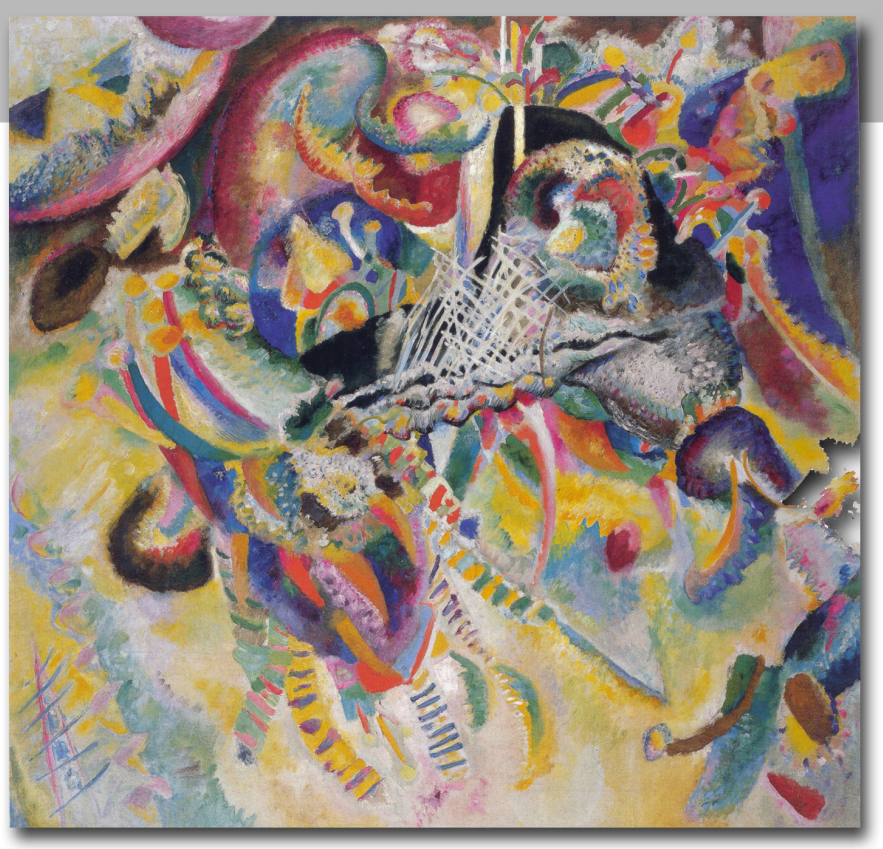




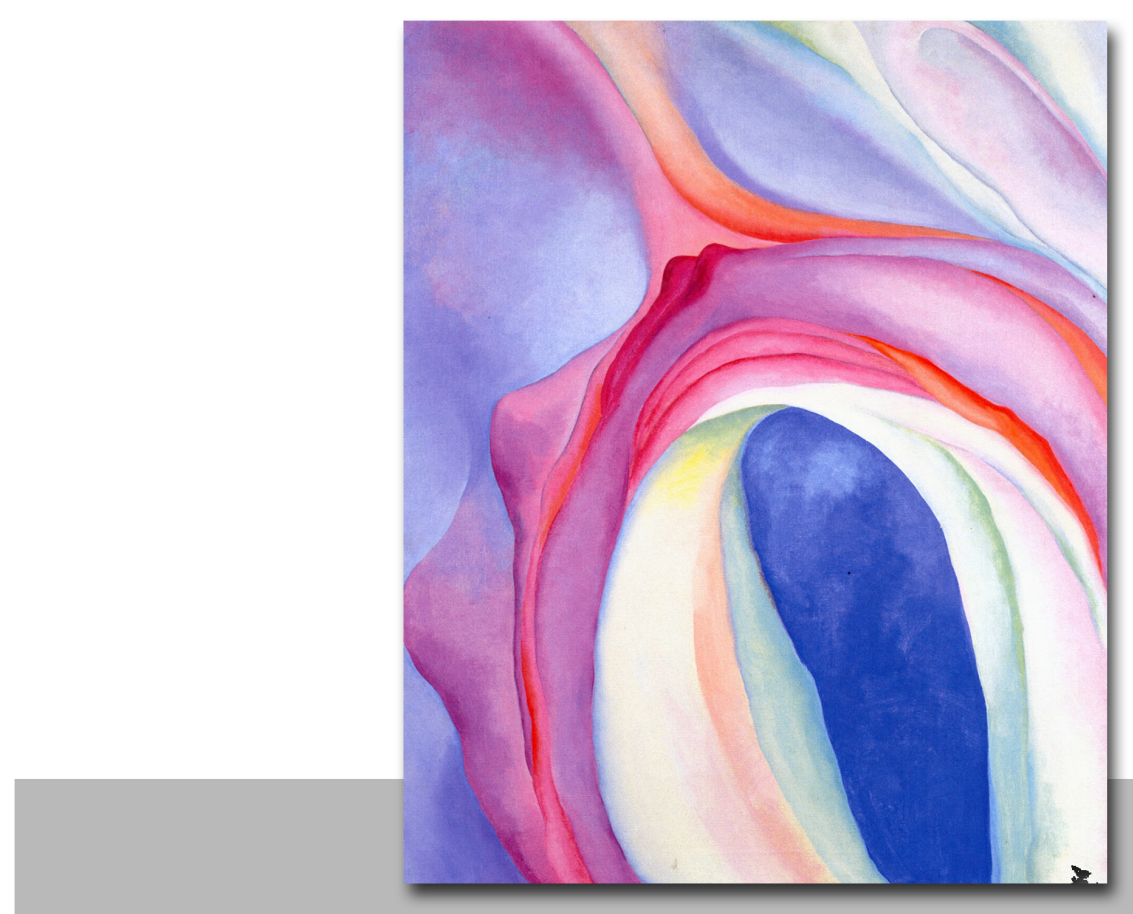

1921 Georgia O'Keeffe - Music-Pink and Blue II

14_17 Stanton Macdonald-Wright

Abstraction on Spectrum (organization 5)

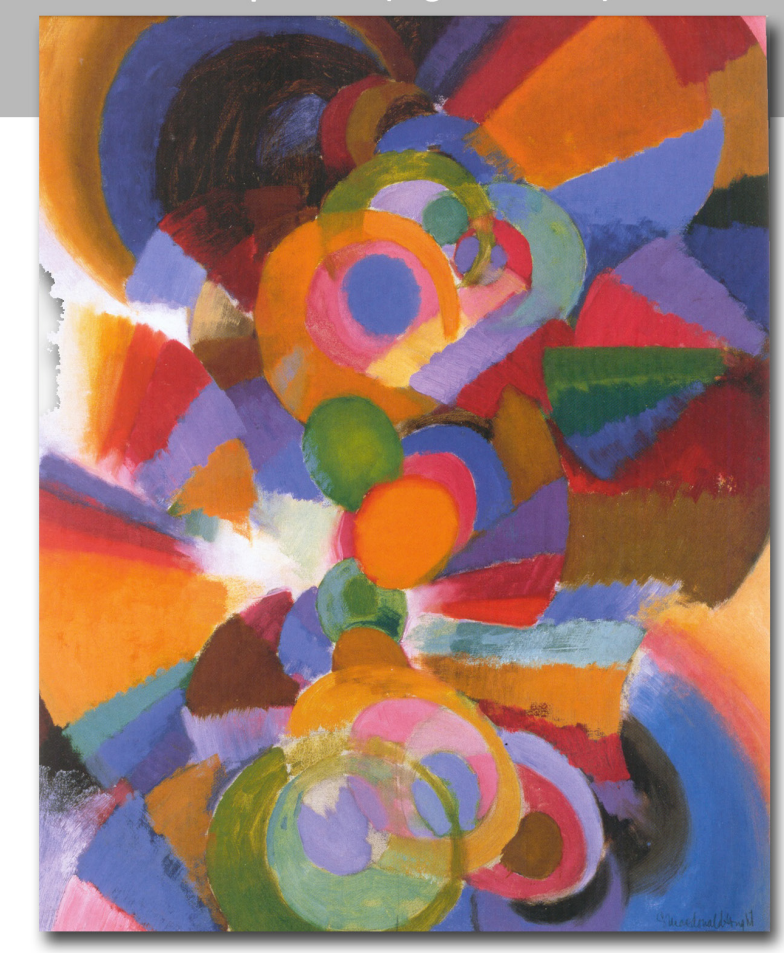

1916 Stanton Macdonald-Wright Synchromy Blue-Green

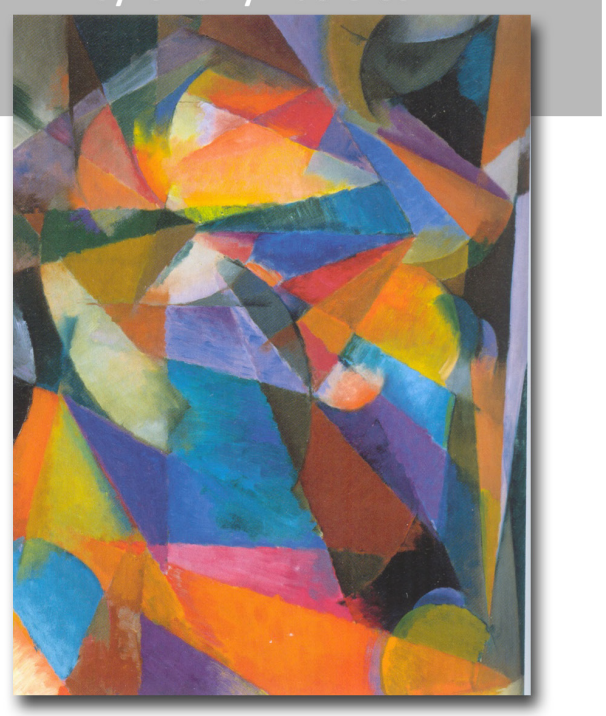




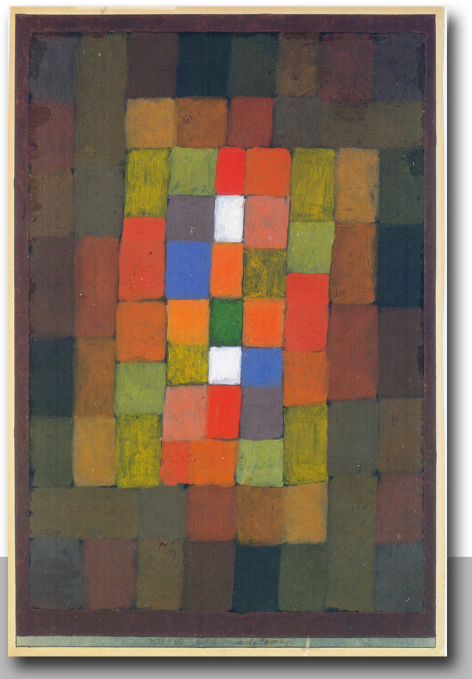

1923 Paul Klee - Static Dynamic Graduation

1921. Paul Klee - Pflanzenwachstum

1927 Wassily Kadinsky - Heavy Circles
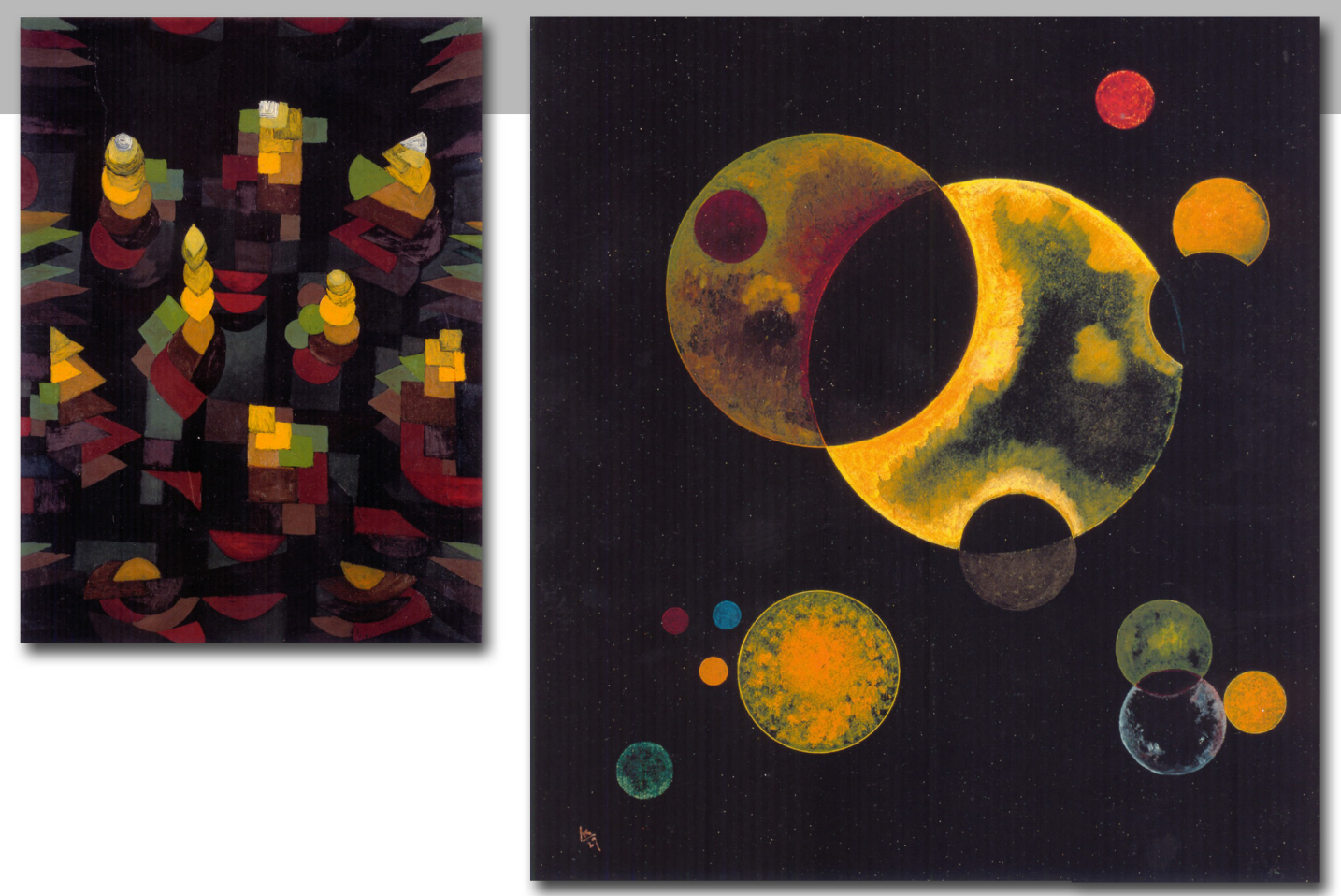


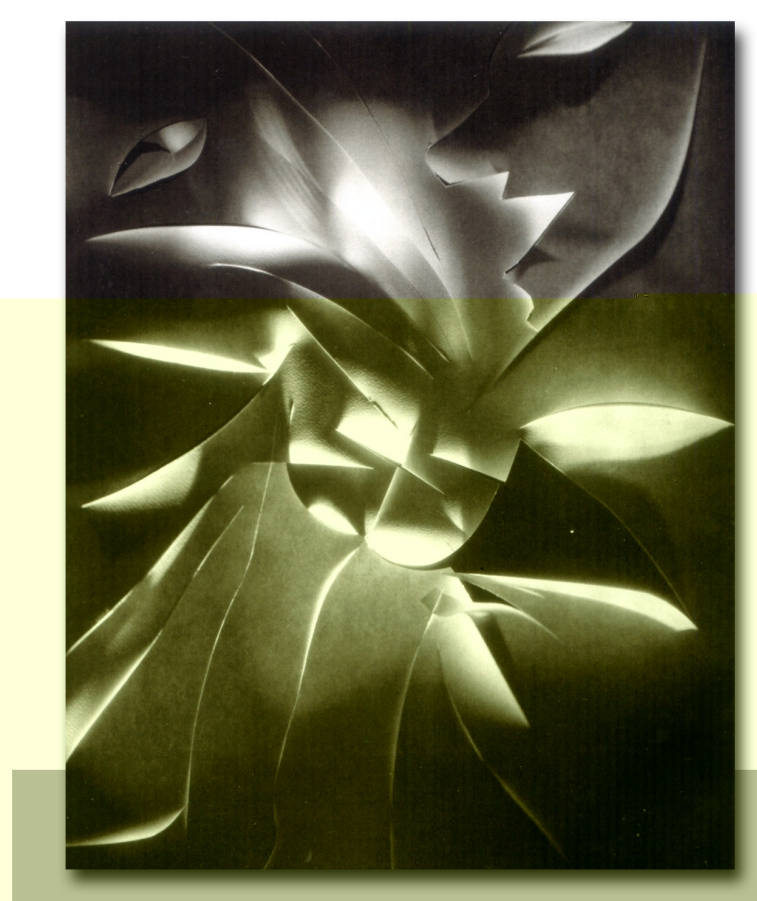

1930 Francis Bruguiere - Design in Abstract Forms of Light \#32 Gelatin-silver Print

1932 Thomas Wilfred - Multidimensional Opus 79
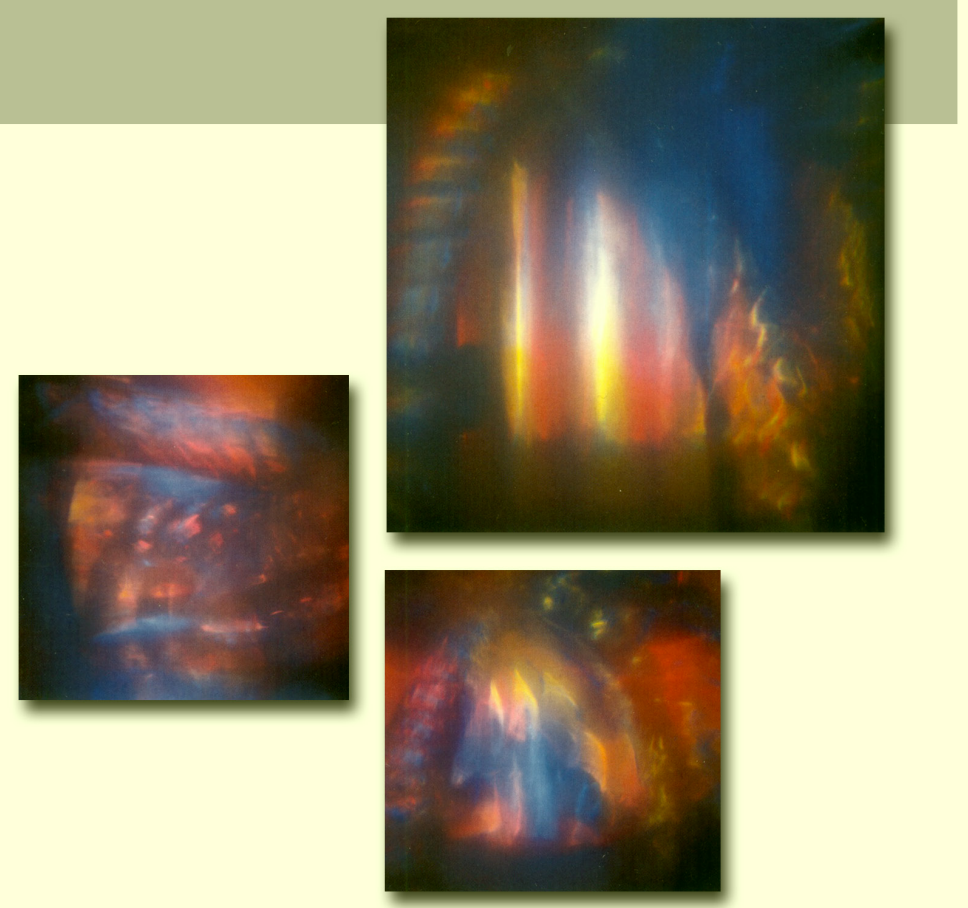

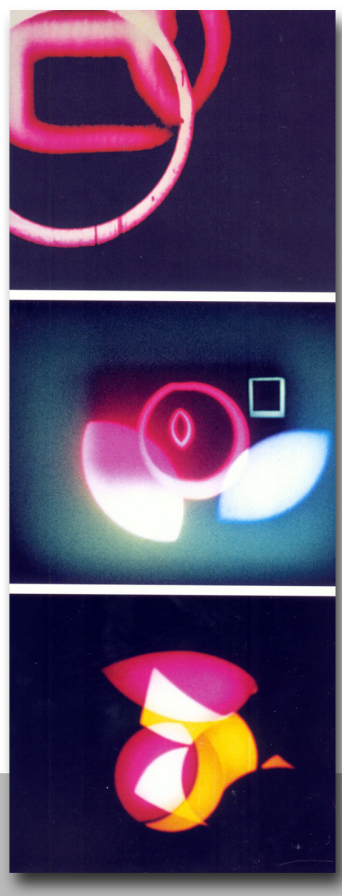

1943_44 John and James Whitney - Stills from Five Film Exercises

1942 Oskar Fischinger - Stills from Radio Dynamics
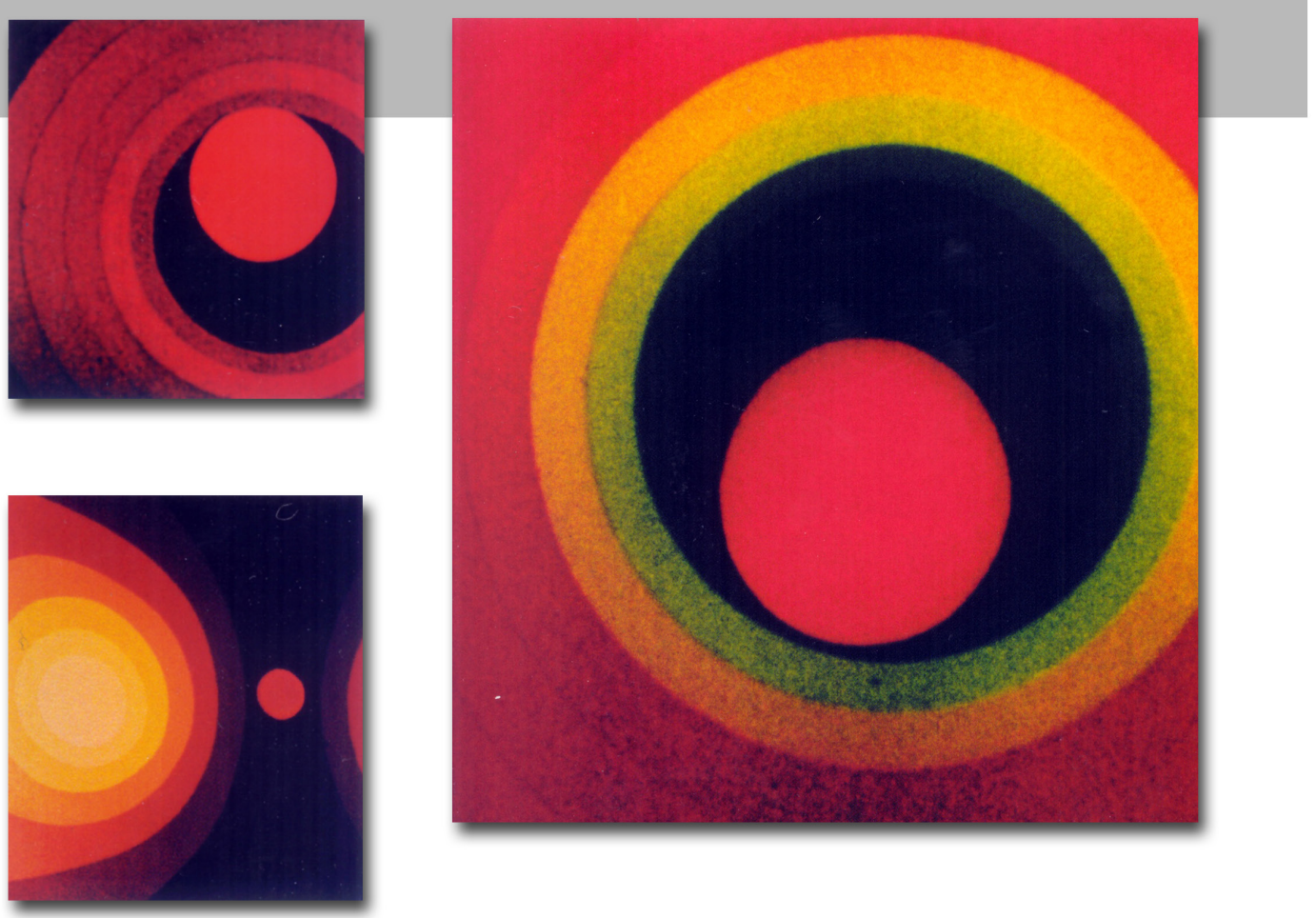

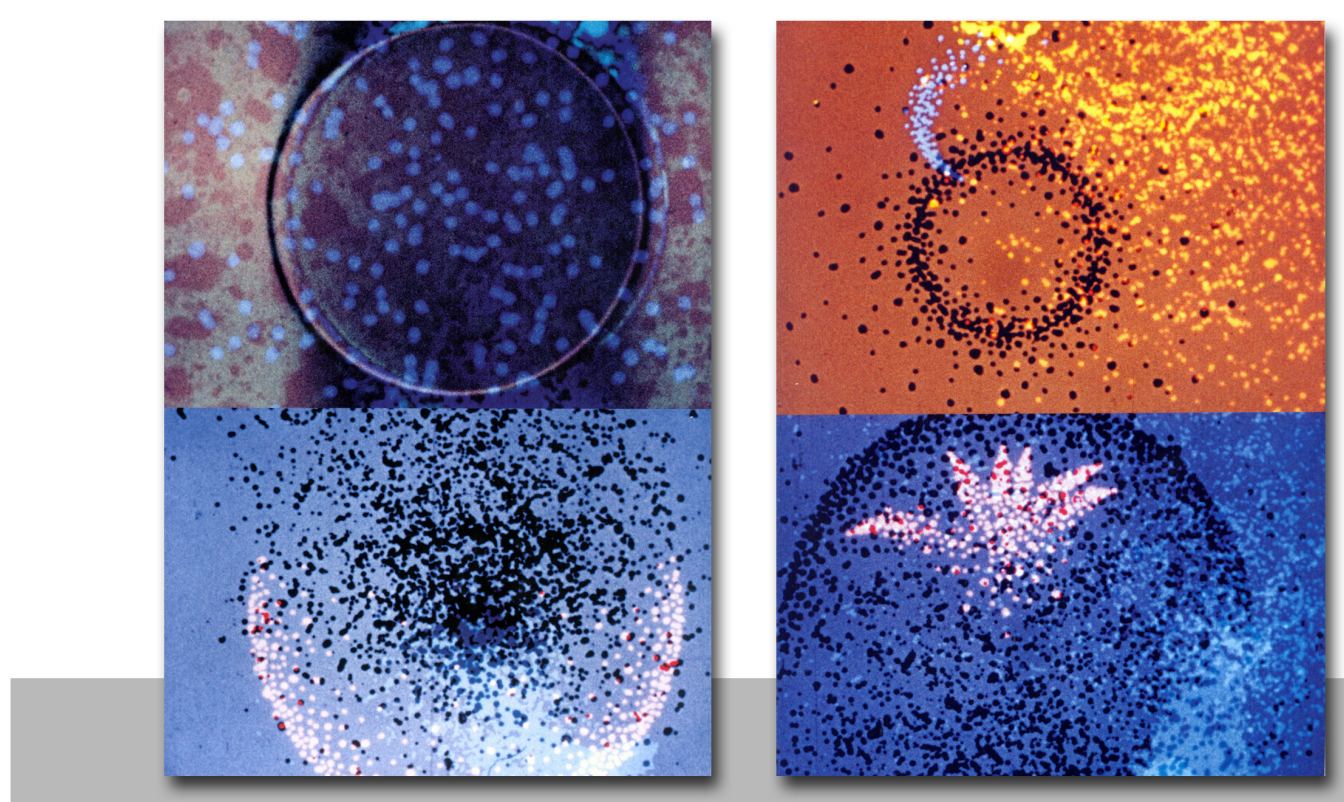

1950_57 James Whitney - Details of Stills from Yantra

1959 Thomas Wilfred - Details of study in Depth Opus 152
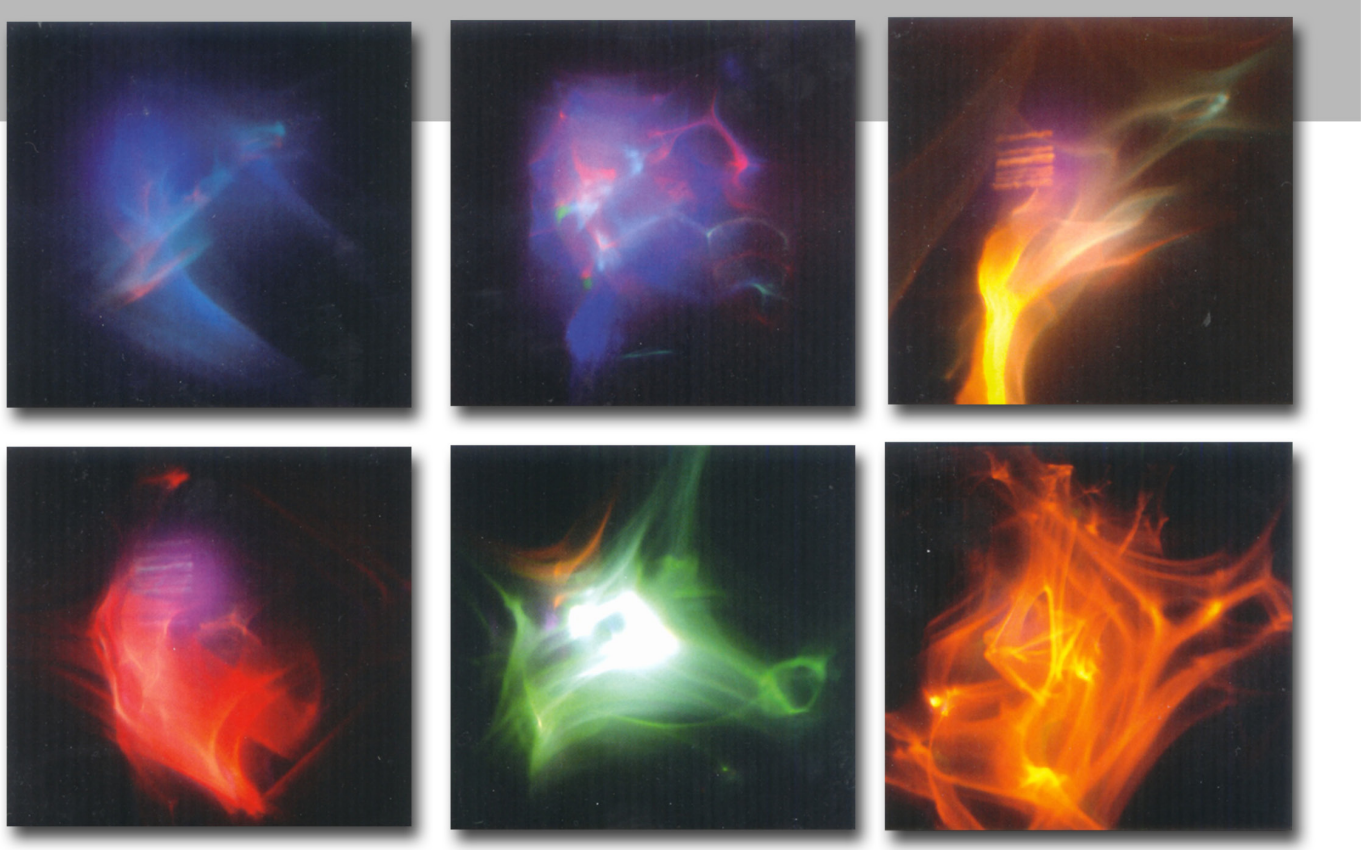


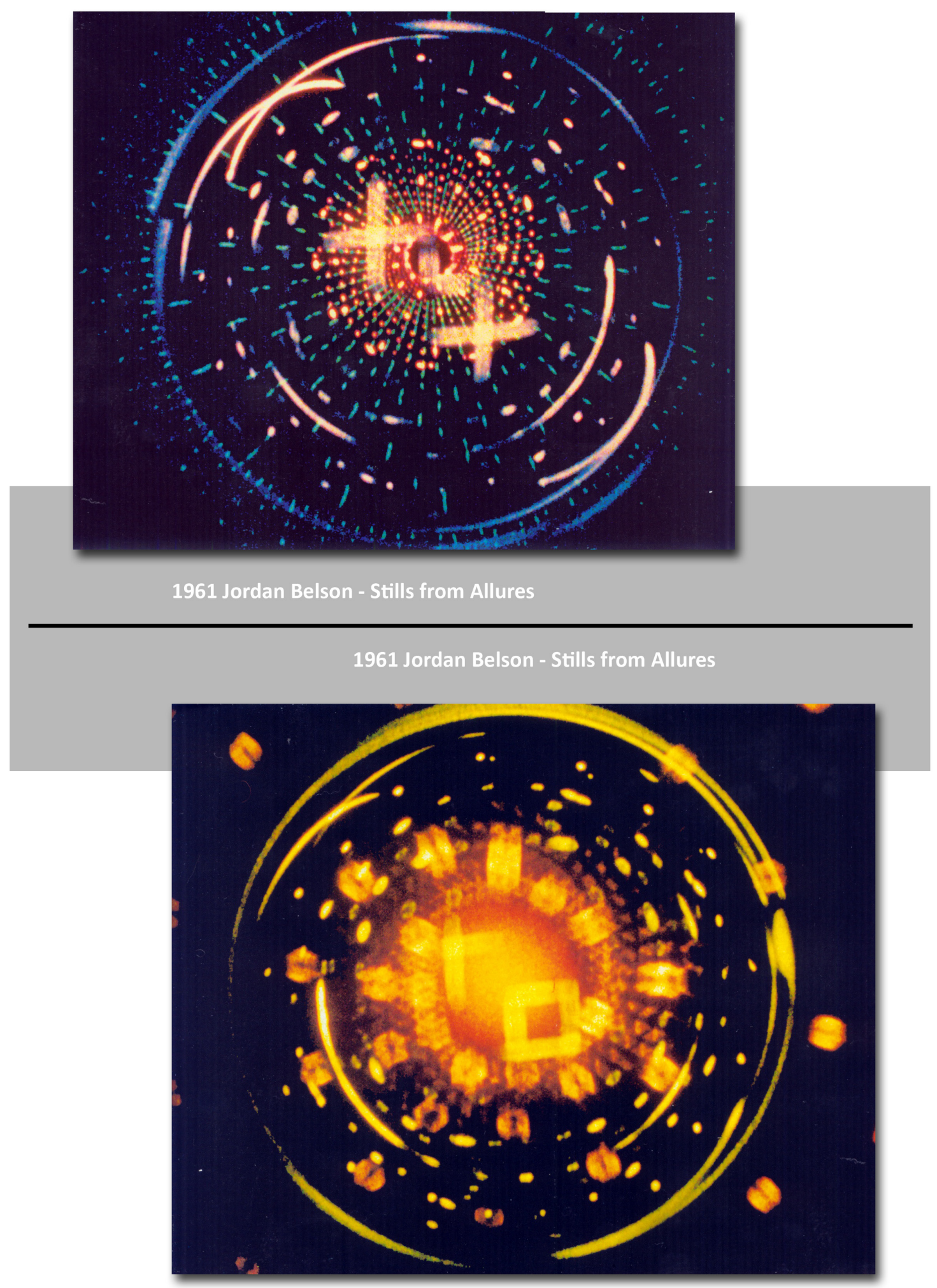




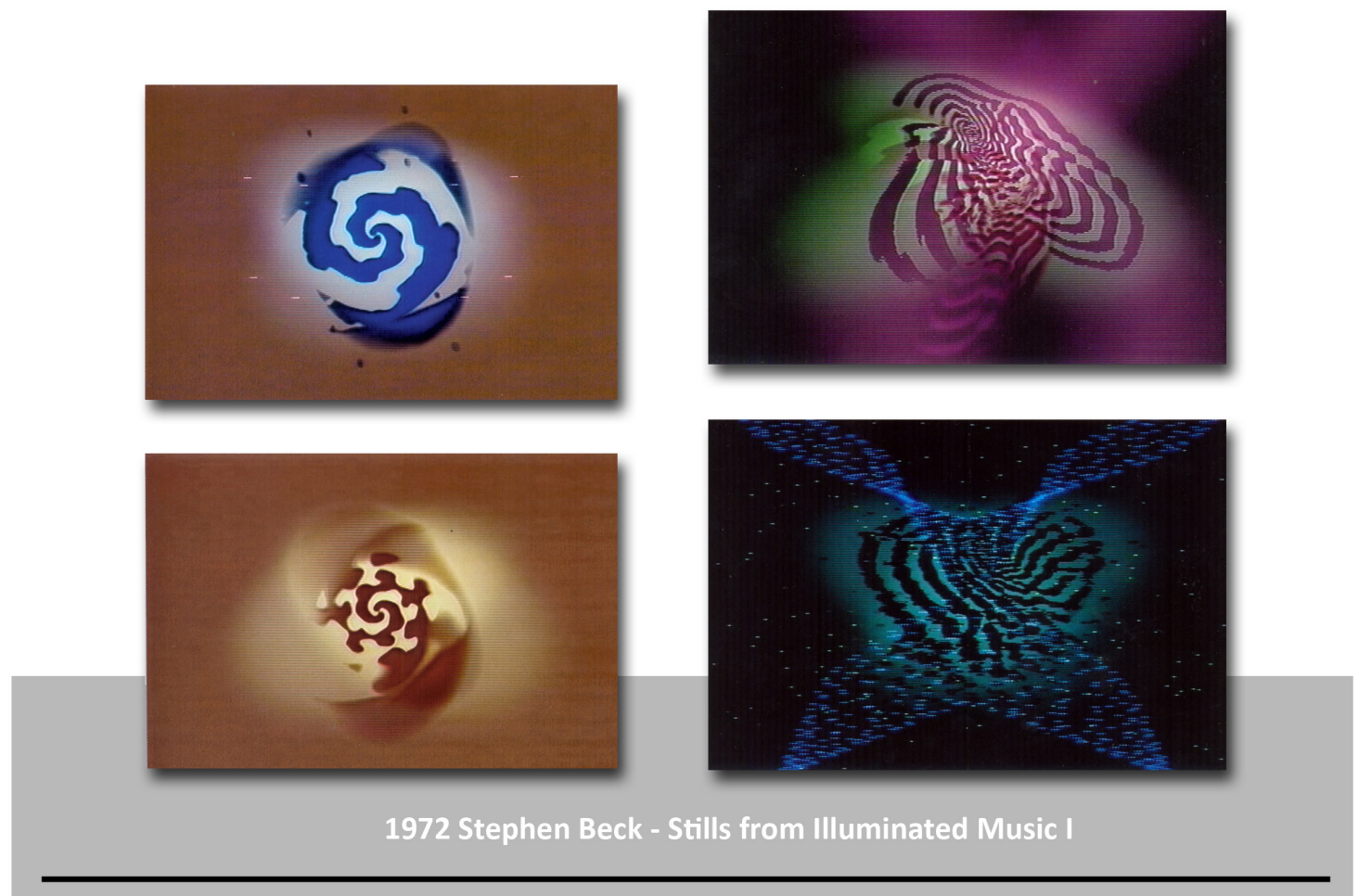

1965 Thomas Wilfred - Untitled Opus 161

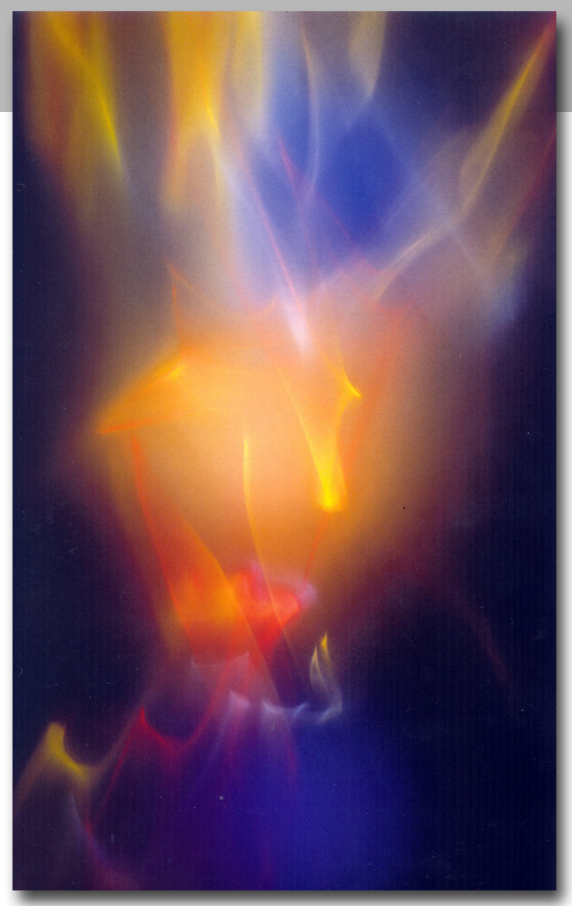


A.x 


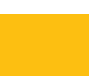




\section{Appendix 2}

\section{Pre-Cinema Instruments}

All the images provided in Appendix 2 are sourced from Burns, Paul. (2009). The History and the Discovery of Cinematography.

Retrieved from: http://www.precinemahistory.net/ and do not belong to the Author of this Thesis 


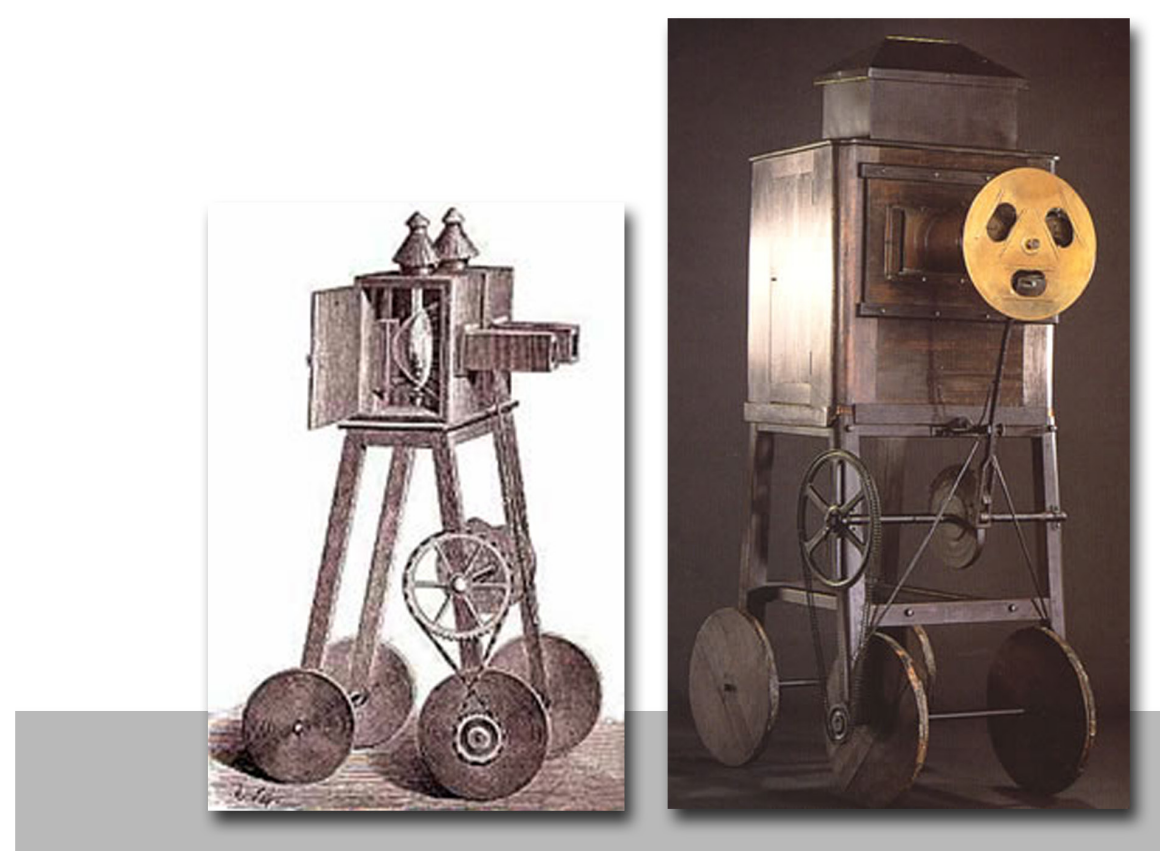

1798 Fantascope - Etienne-Gaspard Robert

1826 Thaumatrope - William Henry Fitton

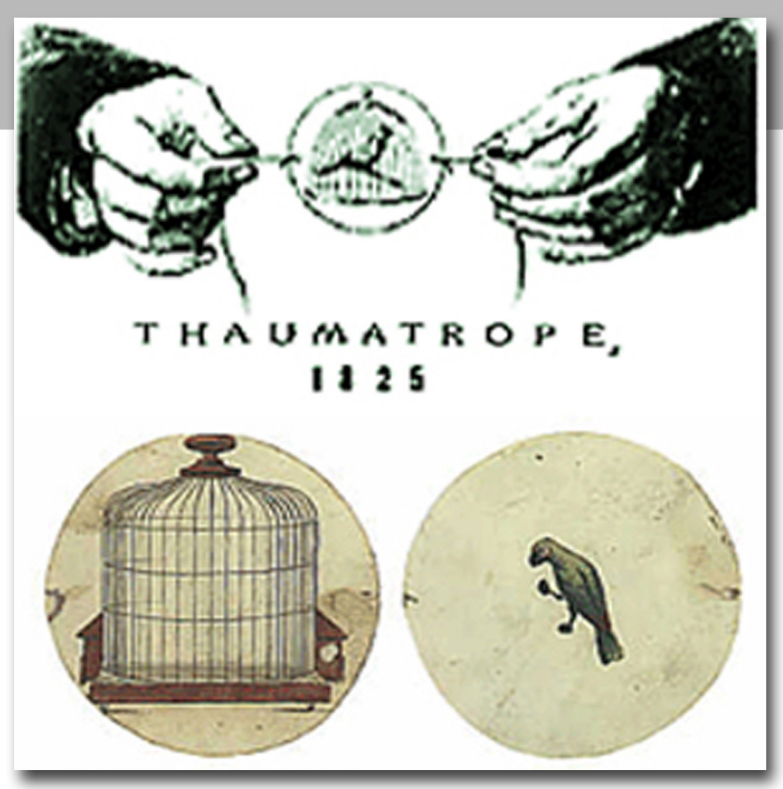



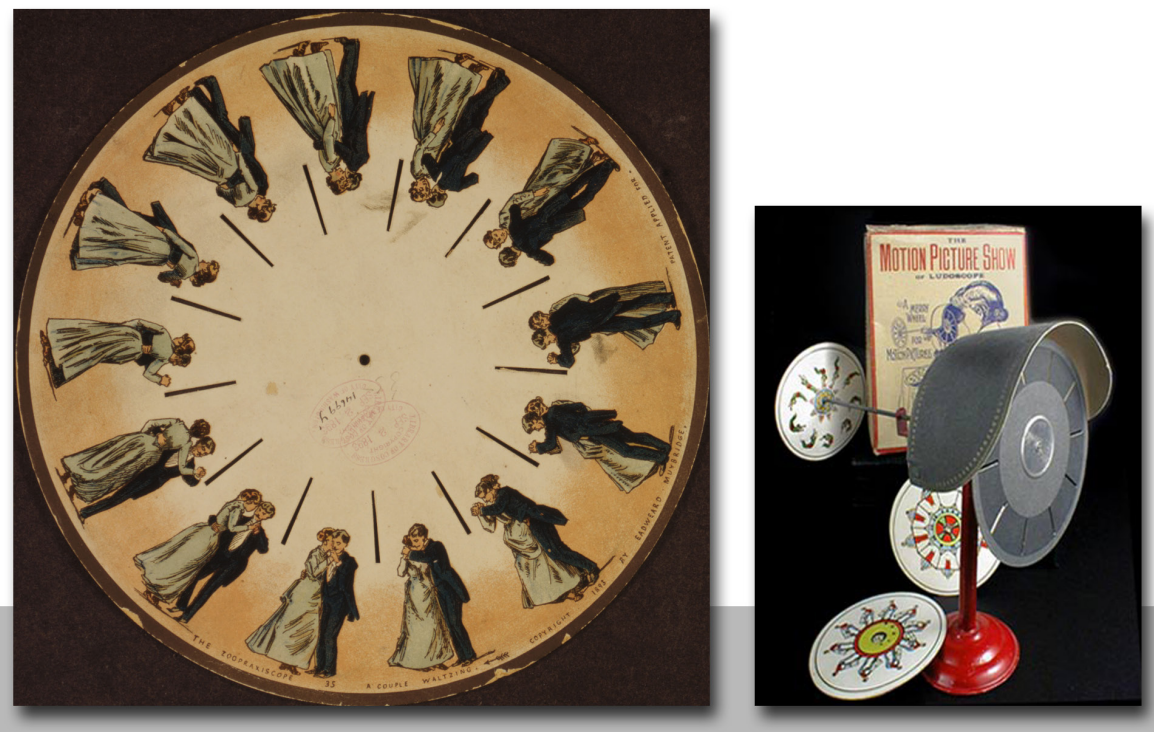

1832 Phenakistoscope - Joseph A F Plateau

1834 Zoetrope - William Horner

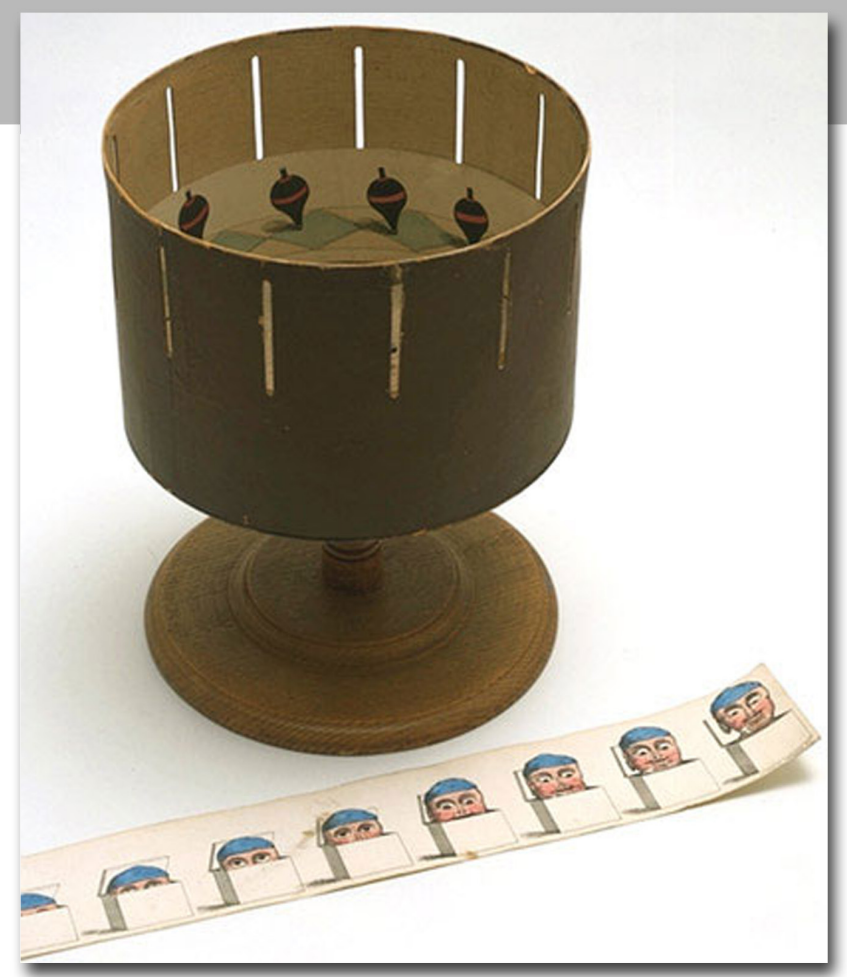




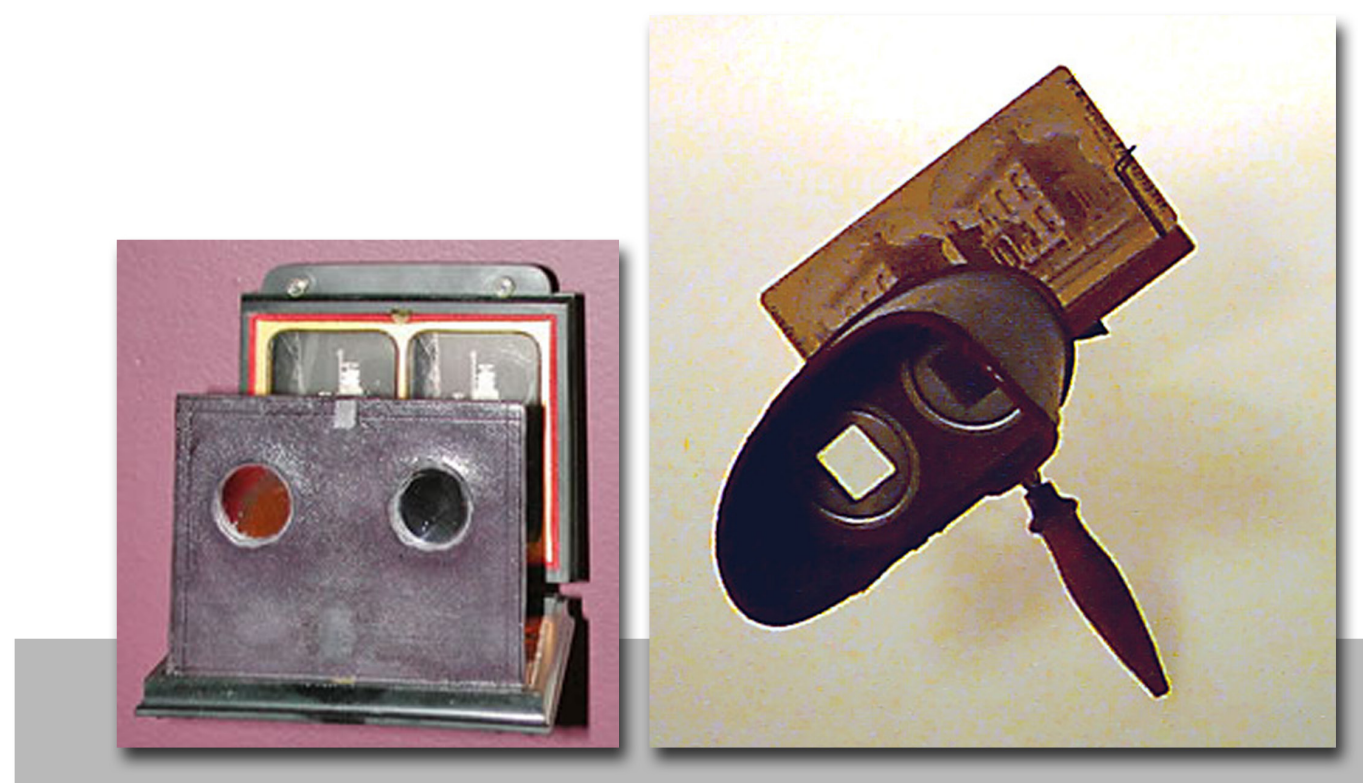

1852 Stereoscope - John Frederick Mascher

1862 Pepper's Ghost - John H Pepper

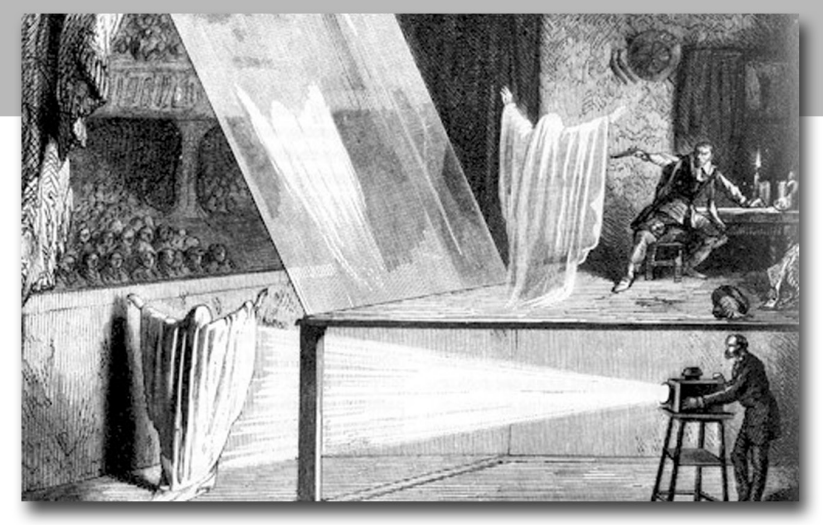




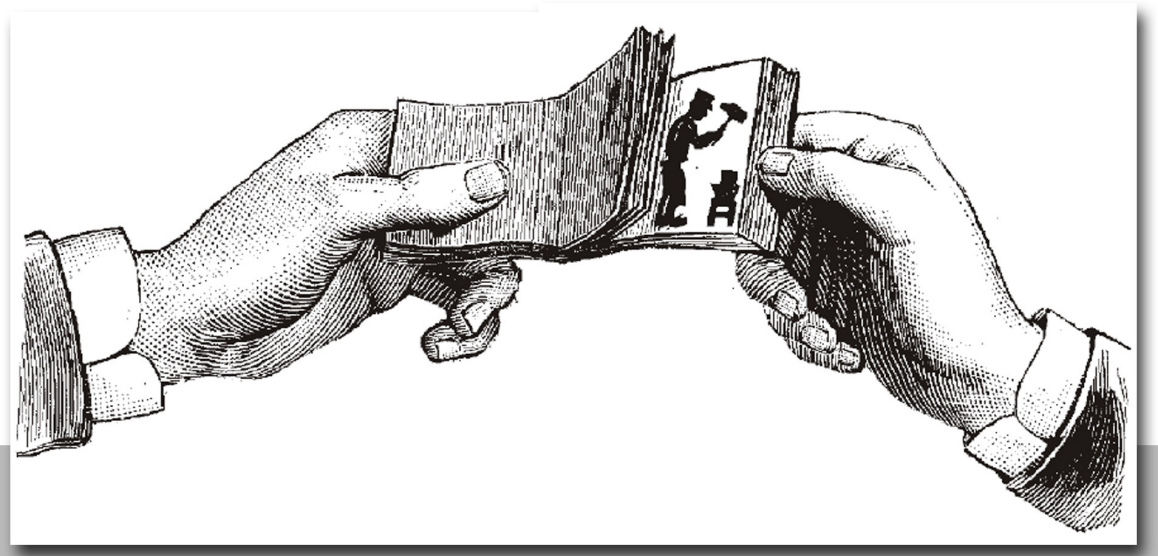

1868 Kineograph - John Barnes Linnett

1867 Zoetrope - William F Lincoln 1868 Mutoscope
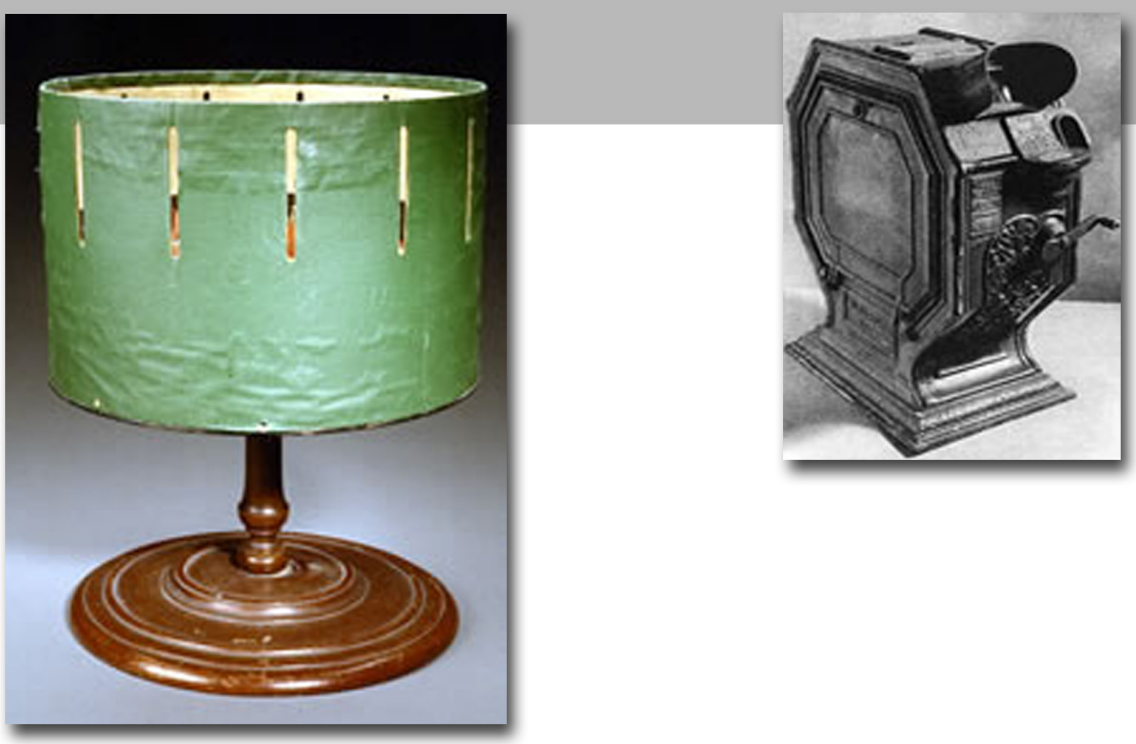


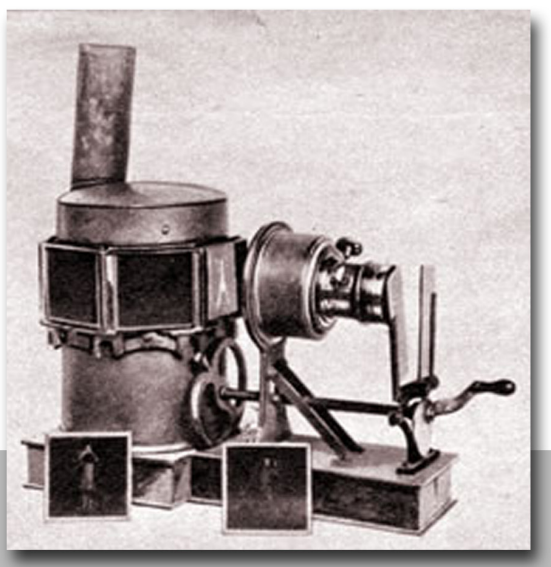

1875 Phantascope - John A R Rudge

1877 Praxinoscope - Emile Reynaud

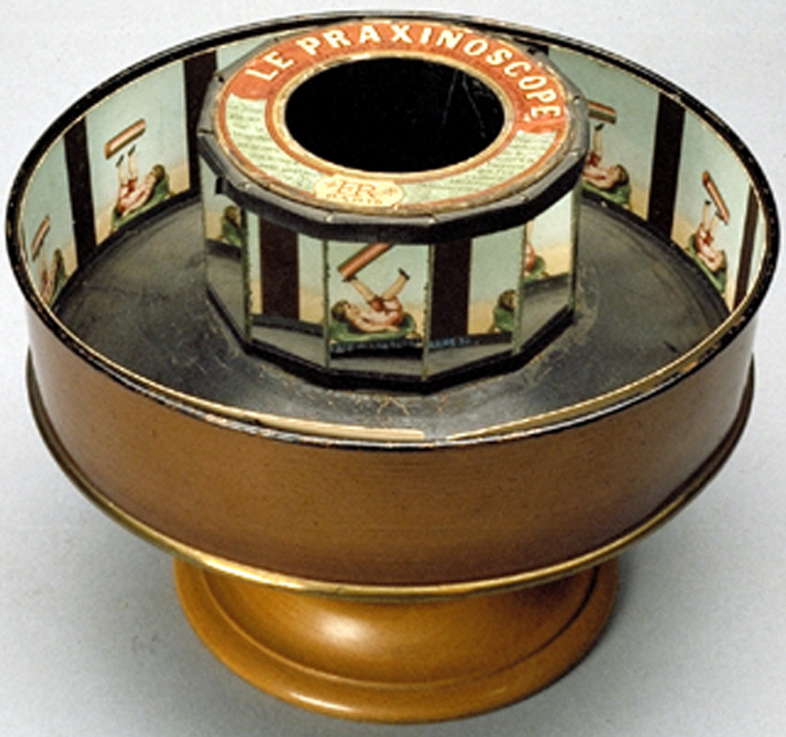




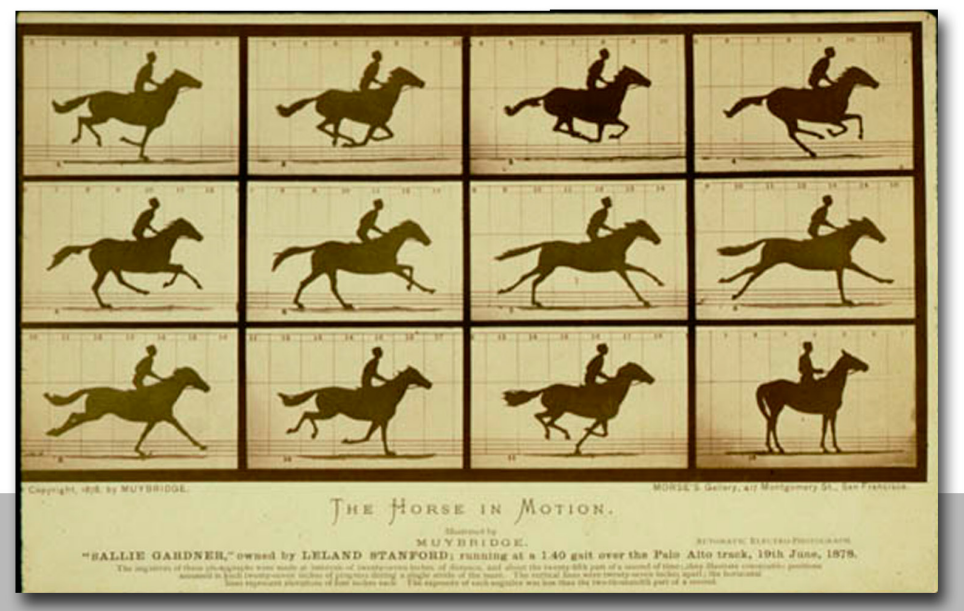

1878 Motion Photography - Edward Muybridge

1878 Praxinoscope - Charles Emile Raynaud

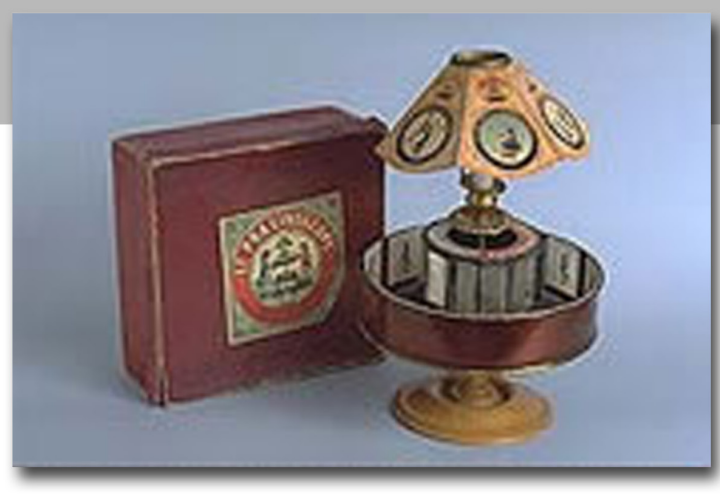




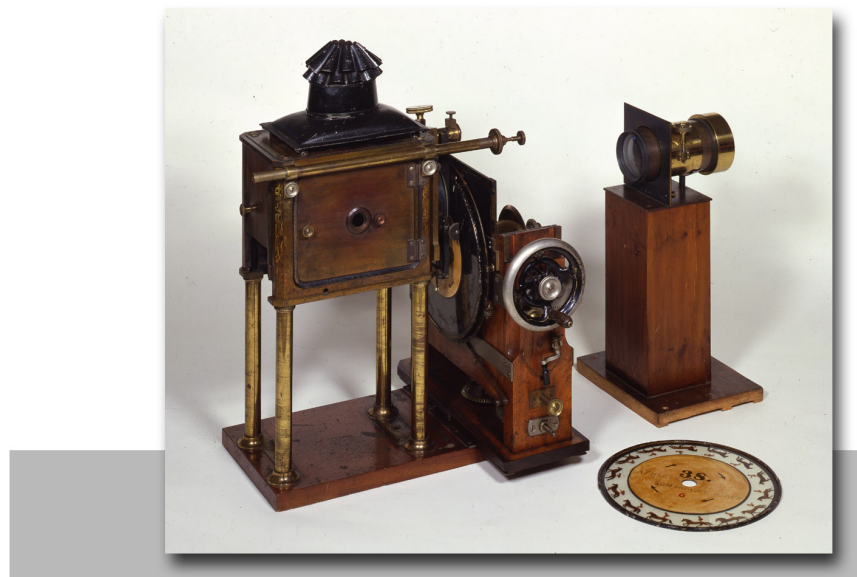

1880 Zoopraxiscope - Edward Muybridge

1882 Fusil Rifle - Etienne-Jules Marey
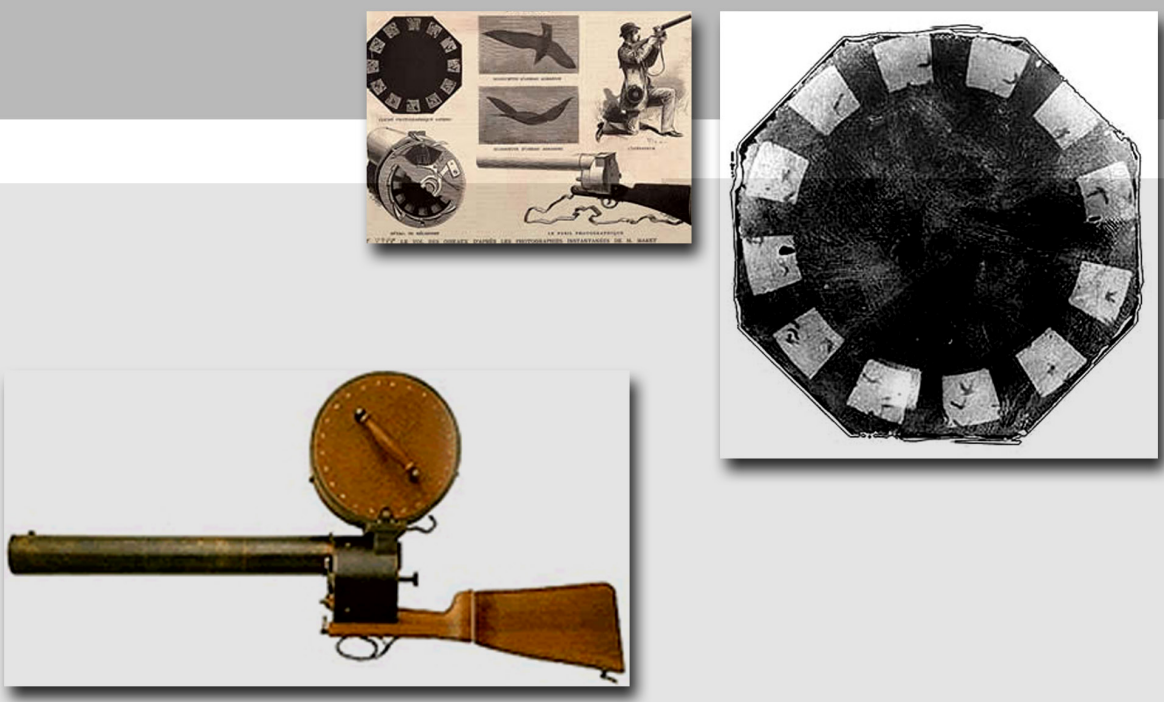


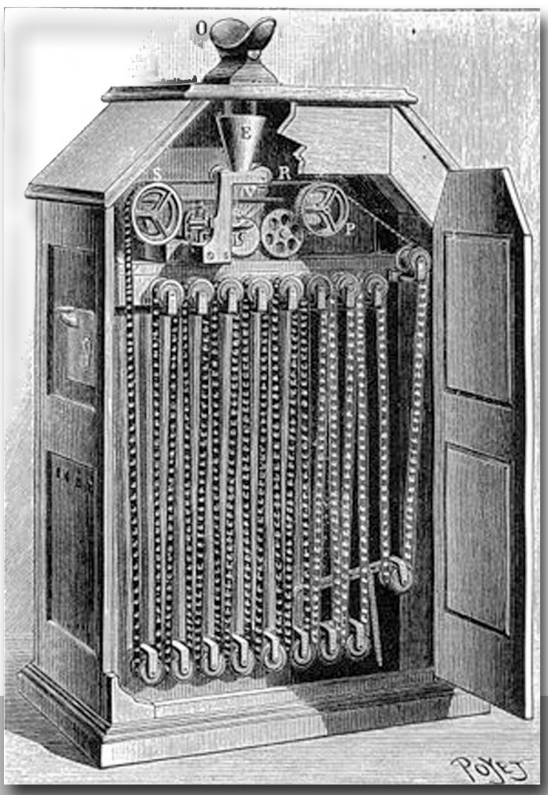

1889 Kinetoscope - Thomas A Edison

1890 Mutoscope - Herman Casler

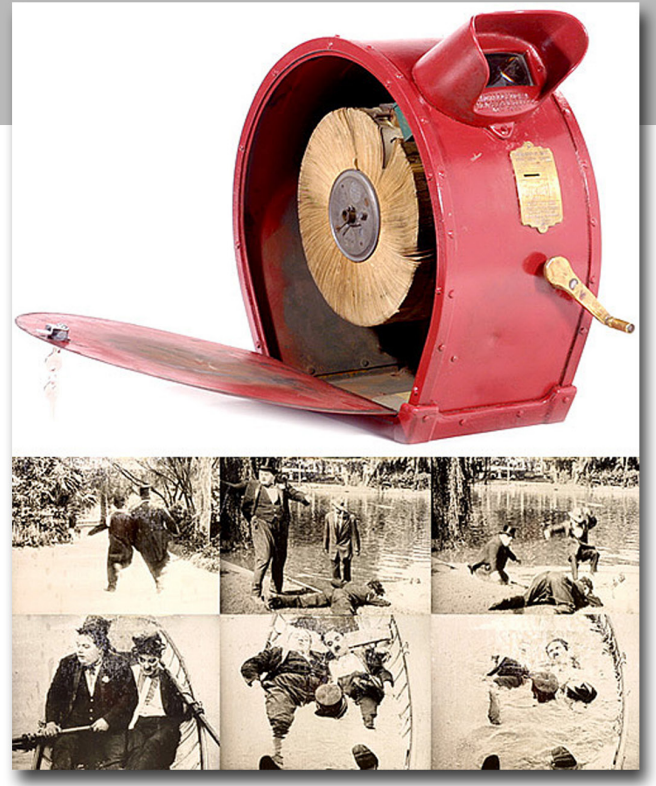




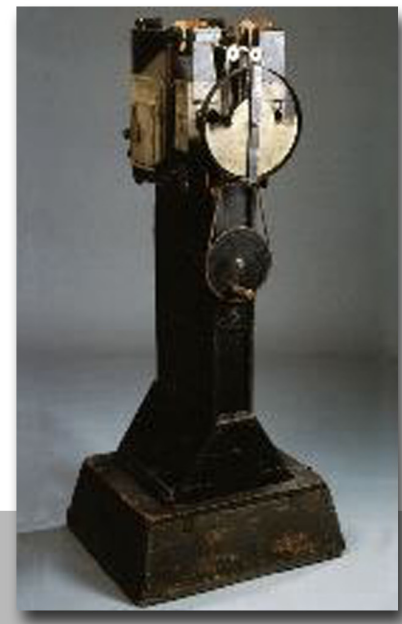

1895 Bioskop - Maximillian Skladanowsky

1896 Vitascope - Thomas A Edison

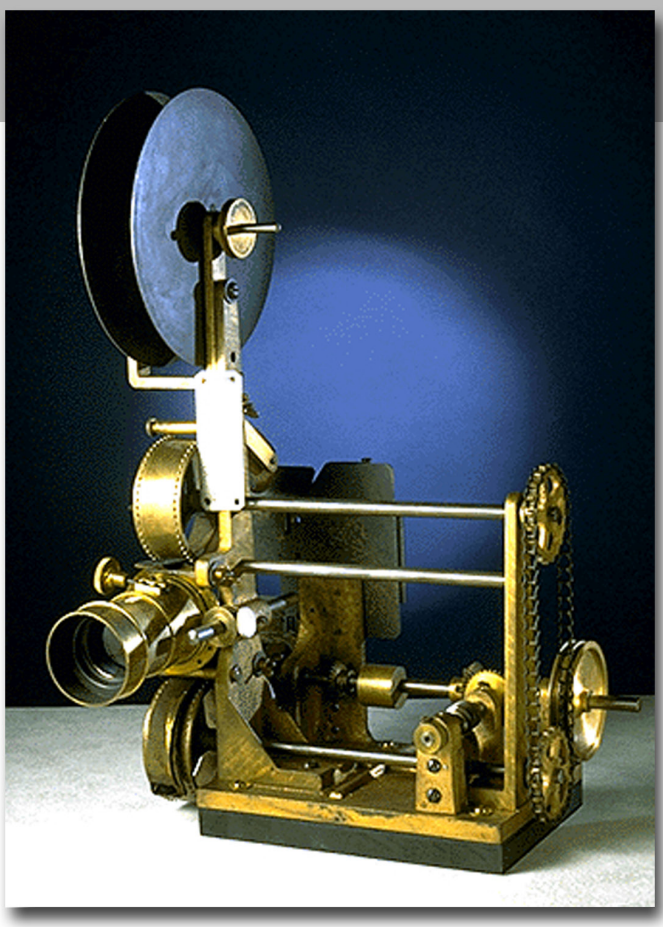


B. $x$ 